\title{
Rotational Velocities and Radii of Pre-Main-Sequence Stars in the Orion Nebula Cluster
}

\author{
Katherine L. Rhode ${ }^{1}$ \\ Department of Astronomy, Yale University, New Haven, CT 06520 \\ rhode@astro.yale.edu \\ William Herbst ${ }^{1}$ \\ Astronomy Department, Wesleyan University, Middletown, CT 06459 \\ bill@astro. wesleyan. edu \\ and \\ Robert D. Mathieu ${ }^{1}$ \\ Department of Astronomy, University of Wisconsin, Madison, WI 53706 \\ mathieu@astro.wisc.edu
}

\begin{abstract}
We have obtained high-dispersion spectra for a sample of 256 pre-main-sequence stars in the Orion Nebula Cluster (ONC) in order to measure their projected rotational velocities and investigate the rotational evolution and physical properties of young, lowmass stars. Half the stars were chosen because they had known photometric periods and the other half were selected as a control sample of objects without known periods from the same portion of the H-R diagram. More than $90 \%$ of the spectra yielded $v \sin (i)$ measurements, although about one-third are upper limits. We find strong evidence confirming the long-held assumption that the periodic light variations of $\mathrm{T}$ Tauri stars are caused by rotation of spots on their surfaces. We find no statistically significant difference between the $v \sin (i)$ distributions of the periodic and control samples, indicating that there is no strong bias in studying the rotational properties of young stars using periodic variables. Likewise, the classical and weak T Tauri stars exhibit $v \sin (i)$ distributions that are statistically the same. For stars with known period and $v \sin (i)$, the mean value of $\sin (i)$ is significantly lower than expected for a random distribution
\end{abstract}

\footnotetext{
${ }^{1}$ Visiting Astronomer, Kitt Peak National Observatory, National Optical Astronomy Observatories, which is operated by the Association of Universities for Research in Astronomy, Inc. (AURA) under cooperative agreement with the National Science Foundation
} 
of stellar rotation axes. This could be caused by errors in one or more of the quantities that contribute to the $\sin (i)$ calculation or to a real physical effect. We investigate the possible causes and find that $\langle\sin (i)\rangle$ has the expected value if we increase the effective temperatures of our stars by $400-600 \mathrm{~K}$. Finally, we have calculated minimum radii $(R \sin (i))$ for stars with both $v \sin (i)$ and period, as well as average radii for objects grouped by their location in the H-R diagram. We find evidence at the three-sigma level that the radii of the stars on similar mass tracks are decreasing as the stars move closer to the ZAMS.

Subject headings: stars: pre-main sequence; stars: rotation; stars: fundamental parameters

\section{Introduction}

T Tauri stars are low-mass, late-type stars that are still undergoing gravitational contraction toward the main sequence. They often show characteristics that reflect the dynamic, turbulent nature of this early stage of evolution: powerful magnetic fields cause prominent dark spots to form on their surfaces, bipolar outflows or jets direct material away from them at high velocities, and accreting matter that is channelled along magnetic field lines produces surface hot spots that give rise to rapid brightness fluctuations (Bertout 1989; Herbst et al. 1994). In addition, many $\mathrm{T}$ Tauri stars have thin circumstellar disks of dust and gas that can extend out to a few hundred astronomical units. Studying the rotation and evolution of these objects can lead to insights into the early evolution of solar-type stars and the planetary systems that form around them.

One of the best regions of the sky in which to study T Tauri stars is the Orion Nebula Cluster (ONC). The ONC is located in the portion of the Orion Nebula centered on four OB-type stars called the Trapezium. The cluster is especially well-suited for investigations of star formation and early stellar evolution because of its large stellar population, the range of stellar masses present $\left(\sim 0.1-50 \mathrm{M}_{\odot}\right)$, and the very young ages $(<1-2 \mathrm{Myr})$ of its member stars (Hillenbrand 1997). In addition, cluster membership for nearly 900 stars in the ONC has been established by a proper motion survey by Jones \& Walker (1988), and Hillenbrand (1997) and Hillenbrand et al. (1998) have published extensive studies of the observable properties of ONC members. These reasons, combined with the relative proximity of the ONC (its distance is $\sim 470 \mathrm{pc}$; Walker 1969), make it a natural choice for detailed studies of the physical and rotational properties of T Tauri stars.

We began such a study in 1997, with the aim of using high-dispersion spectroscopy to measure projected rotational velocities, or $v \sin (i)$ values, for a large sample of pre-main-sequence stars in the ONC. Rotation periods, measured via starspot-modulated light curves, have already been obtained for about 400 stars in the ONC (Herbst et al. 2000; Stassun et al. 1999; Herbst et al. 2001). We sought to combine data obtained via both photometric and spectroscopic techniques in order to investigate the fundamental properties and rotational evolution of young, low-mass 
stars. When we began our study, periods for approximately 130 ONC stars had been measured at Van Vleck Observatory (VVO) on the campus of Wesleyan University (see Mandel \& Herbst 1991, Attridge \& Herbst 1992, Choi \& Herbst 1996). These will hereafter be referred to as the VVO periods. Details about the VVO observing program are described in Herbst et al. (2000); briefly, multiple fields around the $\mathrm{ONC}$ are imaged repeatedly on each of $\sim 30$ clear nights over the course of an observing season, using a 0.6-meter telescope and a CCD. For our $v \sin (i)$ study, we chose as targets a sample of stars with measured VVO periods (hereafter the "Periodic sample"), as well as a comparably-sized sample of ONC stars from the same portion of the H-R diagram without known rotation periods (hereafter the "Control sample"). The properties of these two samples are described in more detail in Section 2.1.

In addition to using our $v \sin (i)$ measurements to investigate pre-main-sequence stellar rotation in the ONC, we had other specific objectives in mind. First of all, measuring $v \sin (i)$ values for stars with known periods can confirm that rotation of surface cool or hot spots is indeed the cause of the periodic variations of $\mathrm{T}$ Tauri stars. It has been assumed since the pioneering studies of Rydgren and Vrba in the 1980's (e.g., Rydgren \& Vrba 1983, Vrba et al. 1986) that the periodic variations of these objects can be interpreted as due to rotation. Stassun et al. (1999) observed a correlation between line width and angular velocity among ONC stars, supporting this assumption. We address the issue further in this paper. Also, for individual stars there is often an ambiguity of a factor of two in the rotation period (i.e., caused by spots on two sides) as well as the possibility of aliasing. Combining $v \sin (i)$ and period measures for a sample of stars of known luminosity and effective temperature can allow us to test the rotational interpretation of periods for pre-main-sequence stars and can help us identify cases of period doubling or aliasing.

Furthermore, for objects with measured rotation periods, we can use their $v \sin (i)$ values to obtain statistical estimates of their radii. With the exception of four known pre-main-sequence eclipsing binaries (Popper 1987; Casey et al. 1995, 1998; Andersen 1991; Mamajek et al. 2000; Covino et al. 2000), we have no direct measurements of the sizes of pre-main-sequence stars. Since $P v \sin (i)=2 \pi R \sin (i)$, combining the stellar rotation period $P$ with $v \sin (i)$ can constrain the stellar radius $R$ to within a factor of $\sin (i)$. The average value of $\sin (i)$ can be calculated; thus average radii can be derived for stars grouped according to their location in the H-R diagram. In combination with luminosities, radii also provide an independent check on effective temperatures, which are typically derived from spectral types by comparing pre-main-sequence stellar spectra with those of main sequence or evolved stars.

Another objective of the study was to determine whether the sample of stars that have persistent spots (and therefore can yield rotation periods) have rotational properties that are representative of the properties of ONC members as a whole. One can imagine that our understanding of pre-main-sequence rotational evolution might be biased by data derived from spotted stars. For example, if rapidly-rotating stars have more active dynamos, they may be more likely to have prominent, persistent spots on their surfaces and thus would be overrepresented in photometric rotation studies. Comparing the $v \sin (i)$ distributions of the Periodic and Control samples provides 
an important observational test of our understanding of the initial conditions of pre-main-sequence rotation.

Here we present the results from our $v \sin (i)$ study of 256 targets in the ONC. The following section contains a summary of the characteristics of the stellar sample and describes the observations and technique used to measure $v \sin (i)$. The results of the study are presented in Section 3 . In that section, we explore the measured $v \sin (i)$ values of various sub-samples of stars, compare our $v \sin (i)$ values to the equatorial velocities calculated for stars with measured period, luminosity and effective temperature, and finally combine period and $v \sin (i)$ data to derive average radii for stars binned according to their location on the H-R diagram. A summary of our findings is given in the last section of the paper.

\section{Observations and Analysis}

\subsection{Characteristics of the Sample}

For information about our target stars, we have relied on two previous studies of the ONC. Jones \& Walker (1988) undertook a proper motion study of the cluster and published membership probabilities for 1053 stars in the region. Throughout this paper, we refer to stars by the number assigned by Jones \& Walker, e.g., JW 100. Hillenbrand (1997) published a comprehensive study of star formation in the ONC, including optical photometric data for 1600 stars and spectra for $\sim 900$ stars. All luminosities, effective temperatures, spectral types, $I$ magnitudes, and $V-I$ colors given in this paper come from the Hillenbrand study.

The initial set of stars observed for this work was selected because the stars had measured VVO periods. After the first observing run (see Section 2.2), a sample of stars without known periods - the Control sample - was chosen. In order to make a useful comparison between the $v \sin (i)$ distributions of the Periodic and Control sample stars, the latter were chosen to match the basic characteristics of the Periodic sample. The properties of the overall stellar sample are as follows (the few stars with characteristics outside those of the larger sample are noted): (1) Spectral types in the range G6 to M6, with the large majority of stars (88\%) in the range K0 to M4. There

are three exceptions: JW 945 (spectral type B6), JW 1033 (spectral type A0-A5), and JW 165 (spectral type A7). (2) $I$ magnitudes from 10-16, and $V-I$ colors ranging from 0.8 to 4.3 . (3) Typical masses (as determined by Hillenbrand (1997) from pre-main-sequence evolutionary tracks) in the range 0.1 to $3 \mathrm{M}_{\odot}$; the median mass is $0.3 \mathrm{M}_{\odot}$. (4) Cluster membership probabilities from Jones \& Walker (1988) equalling $79 \%$ or above for all but four stars. The large majority of stars (78\%) have membership probabilities of $99 \%$. Four stars were observed that have low membership probabilities; two of them (JW 308 and JW 794) had been found to be periodic in the VVO study at the time they were observed, and the other two (JW 3 and JW 1021) have been found to be periodic in a subsequent study (Herbst et al. 2001). All four stars exhibit the low-amplitude, sinusoidal light variations typical of $\mathrm{T}$ Tauri stars. It is possible that they are $\mathrm{T}$ Tauri stars within 
the ONC and that their proper motions are anomalous or in error, or that they are T Tauri stars superimposed on the ONC but not members of the cluster. For three of them (JW 3, JW 308 and JW 1021) we measure heliocentric radial velocities that are within 1-2 standard deviations from the mean of our distribution (at $27 \mathrm{~km} \mathrm{~s}^{-1}, 20 \mathrm{~km} \mathrm{~s}^{-1}$ and $35 \mathrm{~km} \mathrm{~s}^{-1}$, respectively) indicating that these may indeed be ONC members. For JW 794 we measure a radial velocity of $124 \mathrm{~km} \mathrm{~s}^{-1}$, indicating that this may be a true non-member of the cluster.

Because we wished to have roughly equal numbers of objects in the Periodic and Control samples, and VVO periods were known for $~ 130$ stars, we needed observations of a total of approximately 260 targets. We were able to observe 118 stars for the Periodic sample and 138 for the Control sample. Table 1 gives basic information for the 256 stars observed. Columns 1 and 3 give the identification number and membership probability from Jones \& Walker (1988). Column 2 specifies whether the star was originally chosen as part of the Periodic (P) or Control (C) sample. Columns 4, 5, 6 and 7 are, respectively, $I, V-I$, the log of the luminosity, and the log of the effective temperature for the star from Hillenbrand (1997). Columns 8 and 9 are the measured near-infrared excess and the equivalent width of the Ca II $\lambda 8542$ line from Hillenbrand et al. (1998). Column 10 is the measured period of variation of the star from photometric data, and column 11 indicates the reference from which the period was taken ( $\mathrm{V}=\mathrm{VVO}$ data (Herbst et al. 2000), $\mathrm{S}=$ data from Stassun et al. (1999), E = ESO data from Herbst et al. (2001)). Column 12 gives the star's $v \sin (i)$ and estimated error measured in this study, and column 13 lists the projected radius of the star, calculated by combining the $v \sin (i)$ and rotation period (see Section 3.2). Column 14 indicates whether the cross-correlation function showed sufficient structure to indicate that the star may be a double-lined spectroscopic binary (see Sections 2.4 and 3.1 for details). The last column in the table specifies when the star was observed. Stars that initially showed structure in their cross-correlation functions were, when possible, observed more than once.

The relative spatial locations of the stars observed for this survey compared to the overall Jones \& Walker (1988) survey are shown in Figure 1. Small dots in the figure show the locations of the 1053 stars surveyed by Jones \& Walker, and filled circles are the 256 stars observed for this study. The observed sample is spread throughout the area surveyed by Jones \& Walker. The upper left portion of the field that is relatively empty of stars is a region of the ONC that is optically obscured. Figure 2 shows the positions of the target stars on an HR diagram. Stars in the Periodic sample are shown as filled circles and Control sample stars appear as open circles. The pre-main-sequence evolutionary tracks shown in the figure come from D'Antona \& Mazzitelli (1994, model 1). The target stars span the mass range of the tracks and most lie above the $3 \times 10^{6}$-year isochrone. The bulk of the stars are concentrated toward the low-temperature, low-luminosity end of the diagram.

We note that the Control sample stars were chosen without regard to whether they appeared within the dozen or so fields that had been monitored in the VVO program. This means that they are not necessarily non-periodic stars (i.e., we were not selecting against stars with measurable periods), but are simply ONC members with the same basic characteristics as the Periodic sample stars. In that sense they were selected to be a true "control" sample. Since this study was initiated, 
about $30 \%$ of the Control sample stars have had their periods measured. Thus Table 1 contains stars that are marked with a "C" in the Sample column, but also have a measured period listed.

\subsection{Observations}

Because we needed to obtain high-dispersion spectra for a large number of objects over a fairly small region of sky (the $\lesssim 1 / 2$ degree subtended by the ONC) a multi-object spectrograph seemed the ideal instrument for our purposes. The 3.5-meter WIYN telescope ${ }^{2}$ at Kitt Peak National Observatory is equipped with the Hydra Multi-Object Spectrograph, which makes possible simultaneous spectroscopy of $\sim 100$ targets over a 1-degree field. Observations for this study took place at WIYN during three separate observing runs, in January 1997, December 1997 and December 1998.

For all observations we used the Bench Camera, Red Fiber cable, and 316@63.4 Echelle grating. This spectrograph setup gave us fairly high resolution $(R \sim 21,500)$ while still allowing us to observe our faintest targets $(V \sim 18)$ with reasonable integration times. The spectra cover the region 6240 $\AA$ to $6540 \AA$, with $\lambda_{\text {central }}=6400 \AA$, and a dispersion of $0.145 \AA$ per pixel. We chose to work in the red portion of the spectrum because of the colors of our program stars $(V-I=1-4)$, while at the same time avoiding the region of the spectrum around $\mathrm{H} \alpha$, where nebular emission can easily overwhelm the light from the star. The spectral resolution (corresponding to a typical FWHM of the slit profile of 2 pixels) is $13.6 \mathrm{~km} \mathrm{~s}^{-1}$ at $6400 \AA$.

The 256 target stars were observed in five different fiber configurations, so typical configurations had fibers positioned on $\sim 50-60$ objects. Crowding in the ONC and limitations on exactly how Hydra fibers can be positioned prevented us from observing larger numbers of stars in a given configuration. At least 15-20 of the remaining fibers in each pointing were placed on blank sky, to be used for sky subtraction during processing of the spectra. Total integration times for the program star fields ranged from $\sim 3-4$ hours, depending on the magnitudes of the stars in the configuration and the sky conditions. In addition to the program stars, we observed five objects from the Gliese Catalog of Nearby Stars (Gliese \& Jahreiss 1991) with spectral types spanning the range of our targets; the spectra of these stars were used as narrow-lined templates in the crosscorrelation process. Information about the templates is given in Table 2. Daytime sky spectra were also obtained for use as G2V template spectra.

Figure 3 shows examples of narrow-lined template and object spectra obtained for this study. The spectra are arranged in order of increasing rotational velocity, and illustrate the effect that this has on stellar absorption lines. The top spectrum is that of Gliese 144, a K2 dwarf used as a narrow-lined template. Its rotational velocity is below our $v \sin (i)$ measurement limit, and

\footnotetext{
${ }^{2}$ The WIYN Observatory is a joint facility of the University of Wisconsin-Madison, Indiana University, Yale University, and the National Optical Astronomy Observatories.
} 
its lines appear narrow. The spectra in the bottom four panels are target stars with projected velocities ranging from $\sim 20-100 \mathrm{~km} \mathrm{~s}^{-1}$. The spectral lines become broader and shallower with larger $v \sin (i)$. The emission lines present in a few of the spectra are nebular lines at $\lambda 6363.8$ due to $[\mathrm{O}$ I] and $\lambda 6371.3$ due to Si II. Regions of the spectra with emission lines were excluded from the $v \sin (i)$ measurement process (i.e., not included in the cross-correlation) described in Section 2.4.

\subsection{Initial Reductions}

Preliminary data reduction was accomplished with standard IRAF ${ }^{3}$ tasks, e.g., ZEROCOMBINE to create combined bias images, CCDPROC to bias-subtract and trim the object images, dome flats and comparison lamp frames, and FLATCOMBINE to create combined dome flats. Then the IRAF task DOHYDRA was used to perform spectral extraction, flat-fielding, fiber throughput correction, wavelength calibration, and sky subtraction on the object frames. The wavelength calibration was done using observations of a Thorium-Argon comparison lamp. In the sky subtraction step, sky fibers with unusually high signal (due to contamination by an object) were identified and deleted. A combined sky spectrum was then made by averaging the remaining sky spectra and using a sigma-clipping algorithm to eliminate cosmic rays. We note that the sky fibers placed randomly around the field will collect flux from both the sky and the Orion nebula. The sky values varied spatially, reflecting variations in the nebular continuum. Although the sky level in the vast majority of fibers varied by $\lesssim 10 \%$, a few had deviations of as much as $30 \%$. A series of tests was performed that indicate that a variable background does not affect the final measured $v \sin (i)$ values. The template star and daytime sky spectra were reduced in the same manner as the target star spectra, with the only difference being that sky subtraction was not executed on the daytime sky frames.

Once DOHYDRA had been run on the object frames, individual integrations taken of the same field (within a given observing run) were scaled and then combined into a single frame using the task SCOMBINE, to remove cosmic rays and produce a single, high signal-to-noise observation. Because the signal degrades at the ends of some of the images, the frames were clipped so that the spectra cover the region between 6275 and $6525 \AA$. All stellar spectra were continuum-normalized before the cross-correlation step was executed.

\subsection{Measuring Radial and Rotational Velocities}

Rotational and radial velocities were derived using the IRAF task FXCOR, which performs Fourier cross-correlation of an object spectrum against a specified template. Figure 3 shows that

\footnotetext{
${ }^{3}$ IRAF is distributed by the National Optical Astronomy Observatories, which are operated by the Association of Universities for Research in Astronomy, Inc., under cooperative agreement with the National Science Foundation.
} 
increasing rotational velocity causes stellar absorption lines to become broader and more shallow. When a stellar spectrum with rotationally-broadened lines is cross-correlated against a narrow-lined spectrum, the width of the cross-correlation peak is sensitive to the amount of broadening present in the first spectrum. Thus, by measuring the width of the peak, one can obtain a measurement of the rotational velocity of the star.

To measure $v \sin (i)$ for our target stars, we began by calibrating the relationship between the width of the cross-correlation peak and $v \sin (i)$. To do this, a narrow-lined template spectrum was artificially "spun up" to mimic that of a higher-velocity star by convolving it with a theoretical rotation profile (Gray 1992). A series of such broadened spectra was created for $v \sin (i)$ values spanning the expected range of observed values. Each broadened spectrum was then cross-correlated against the original narrow-lined spectrum, and the FWHM of the cross-correlation peak was measured. The resultant relationship between $v \sin (i)$ and the FWHM of the cross-correlation peak was fit with a 4th-order polynomial. This process was executed for each of the six template stars (including the Sun). Figure 4 shows, as an example, the calibration curve data produced by cross-correlating broadened versions of the spectrum of the template star Gliese 114 against the unbroadened spectrum. The points on the figure are the data and the dotted line is the polynomial fit to the data. Below $7 \mathrm{~km} \mathrm{~s}^{-1}$, the relationship between the FWHM of the cross-correlation peak and $v \sin (i)$ is flat, but from $7 \mathrm{~km} \mathrm{~s}^{-1}$ upward it is fairly linear. Figure 4 illustrates the absolute lower limit on a $v \sin (i)$ measurement using our analysis method, under ideal circumstances.

The actual limit to which we can measure $v \sin (i)$ depends in part on the size of the slit (fiber) image. With the Bench Camera and our spectrograph setup, the slit image width (as determined by measuring the FWHM of emission line profiles in the comparison lamp spectra) varied from, typically, 1.5 to 1.7 pixels toward the ends of the spectra to $\geq 2$ pixels in the middle (in the worst cases, small portions of some of the spectra have FWHM as large as $2.8-2.9$ pixels). This makes determining the exact limit on $v \sin (i)$ measurement more difficult than if the focus were uniform across the CCD chip. In the regime in which the broadening of a spectral line can be measured (i.e., once the width due to intrinsic broadening is larger than the slit width), $v \sin (i)$ is related to the half-width at zero intensity (HWZI) of the line. The base of the line, or full-width at zero intensity (FWZI), is on the order of two times the FWHM of the line, which means that HWZI is approximately equal to FWHM. The FWHM of the slit image varies in our spectra from $<2$ pixels to $>2$ pixels. Spectral lines from all of these regions contribute to the cross-correlation. In places where, for example, the FWHM is 1.7 pixels, the base of a spectral line spans 3-4 pixels and therefore FWZI is resolved. In such circumstances $v \sin (i)$ values somewhat below the 2-pixelFWHM spectral resolution of $13.6 \mathrm{~km} \mathrm{~s}^{-1}$ can be measured.

Because of the focus variation we cannot determine with certainty what the limit on measuring $v \sin (i)$ is on a star-by-star basis. Our calibration curve suggests that we are sensitive to rotational broadening as small as $7 \mathrm{~km} \mathrm{~s}^{-1}$. However, without observations of $v \sin (i)$ standards in that regime, we are not comfortable reporting $v \sin (i)$ measurements much below our spectral resolution. Thus we take our limit on $v \sin (i)$ measurement to be $11 \mathrm{~km} \mathrm{~s}^{-1}$, since this corresponds to a best-focus 
value of between 1.6 and 1.7 pixels (FWHM). We emphasize that reported values at or somewhat above this limit should be treated with caution. In some cases these values could be upper limits rather than actual velocity measurements.

Once the relationship between the width of the cross-correlation peak and $v \sin (i)$ was established for the narrow-lined templates, each program star spectrum was cross-correlated against one of the template spectra. The template star closest in $T_{\text {eff }}$ to the object was used as the crosscorrelation template. The FWHM of the cross-correlation peak was measured and the $v \sin (i)$ value for the target star calculated using the derived relationship between those two quantities. Radial velocities for the target stars were obtained as part of the cross-correlation step. Radial velocities used for the template stars come from the Gliese catalog (Gliese \& Jahreiss 1991).

In addition to being useful for measuring radial and rotational velocities, the cross-correlation function can also show structure that may indicate that a star is a double-lined spectroscopic binary (SB2). The peak of the function, instead of appearing Gaussian in shape, may look like two Gaussians superimposed on each other, due to the presence of two sets of lines in the stellar spectrum. Figure 5 shows example cross-correlation functions for two stars. The top panel of the figure is the peak of the cross-correlation function for JW 790. The peak is fairly regular and nearly Gaussian in shape. The bottom two panels are the cross-correlation function peaks for JW 669, made using spectra from January 1997 and December 1997. The structure in this star's cross-correlation peak indicates that it may be an SB2.

\section{Results}

\subsection{Rotational Velocities}

Of the 256 stars observed for this study, 18 did not yield reliable $v \sin (i)$ values, primarily because their spectra lacked sufficient signal. In these cases, there was either no obvious peak present in the cross-correlation function, or the peak found by the FXCOR task was not far enough above the noise to be believable. Eighty-three of the remaining 238 stars have $v \sin (i)$ less than or equal to our estimated limit of $11 \mathrm{~km} \mathrm{~s}^{-1}$. The measured $v \sin (i)$ values for our program stars are given in Table 1. For the 18 stars for which no $v \sin (i)$ was measured, "......." is given in the $v \sin (i)$ column; for stars with $v \sin (i)$ below our estimated limit, " $\leq 11.0$ " is given in that column.

Seven stars had sufficient structure in their cross-correlation functions to indicate that they may be SB2s. The cross-correlation peaks for these stars are shown in Figures 5 and 6 . Choosing SB2 candidates was a fairly subjective process; we simply examined the cross-correlation peaks and noted those that were shaped more like two (or more) Gaussians superimposed than like a single, smooth Gaussian curve. Other stars also showed structure in their cross-correlation peaks, in the sense that the peaks appeared noisy and irregular rather than smooth, but we did not mark a star as an SB2 candidate unless its peak looked like multiple Gaussians. The seven SB2 candidates 
are indicated in Table 1 with a "Y" in the "SB2?" column. If any of them are in fact SB2s, their $v \sin (i)$ values would likely be too large, since some of the width of the cross-correlation peak would be due to the fact that their spectra contain two sets of lines.

We have calculated uncertainties on our $v \sin (i)$ values by making use of the parameter $r$ from Tonry \& Davis (1979). It provides a measure of the signal-to-noise of the cross-correlation peak; specifically, $r$ is the ratio of the height of the cross-correlation peak to that of the average peak in the noise component of the cross-correlation function. Tonry \& Davis (1979) showed that the errors in velocities determined via cross-correlation should be proportional to $(1+r)^{-1}$. Hartmann et al. (1986) used cross-correlation to measure $v \sin (i)$ for stars in Taurus-Auriga and Orion, and investigated their observed errors as a function of $r$. They found that the quantity $\pm v \sin (i) /(1+r)$ provided a good estimate for the $90 \%$ confidence level of one of their $v \sin (i)$ measurements.

We have adopted the value $\pm v \sin (i) /(1+r)$ as a reasonable estimate of the one-sigma errors on our $v \sin (i)$ values. We can check this by comparing $v \sin (i)$ values for the eight stars for which we have repeated observations. Two of the stars, JW 2 and JW 433, have $v \sin (i)$ values below our estimated measurement limit. For the remaining six stars, the percentage difference between the $v \sin (i)$ measurements from different observing runs ranges from $4 \%$ to $24 \%$, with an average difference of 13\%. (We note that two of these six stars, JW 99 and JW 669, are SB2 candidates, so the widths of their cross-correlation peaks may change due to orbital motion of the member stars, if they are indeed binaries. However, the percentage differences between the $v \sin (i)$ values from their observations, at $14 \%$ and $4 \%$, respectively, are consistent with the differences for the other stars.) The relative $v \sin (i)$ errors calculated using the $r$ parameter for the six stars range from $10 \%$ to $26 \%$, with an average error of $20 \%$. Furthermore, when we compare the observed percentage difference in $v \sin (i)$ to the relative $v \sin (i)$ errors calculated from $r$ on a star-by-star basis, we find that the uncertainty calculated from $r$ is equal to or larger than the observed difference for all six of the stars. Thus the average error calculated from $r$ is comparable to or larger than our estimated precision based on repeated measurements. The quantity $\pm v \sin (i) /(1+r)$ is given as the error on $v \sin (i)$ in Table 1 . For the stars with repeated measurements, the $v \sin (i)$ and error given in Table 1 are the weighted mean and its associated error, calculated from the multiple measurements.

In the absence of a larger number of cases in which stars had repeated observations taken during separate observing runs, we can perform another test of whether our $v \sin (i)$ error estimates are appropriate. As explained in Section 2.3, multiple observations of a given Hydra field taken during the same observing run were combined to remove cosmic rays and create a single, high signalto-noise observation. A useful comparison to make is to take these individual Hydra frames and measure $v \sin (i)$ for the stars appearing in them. This is equivalent to having multiple, independent observations of the stars in these fields. Moreover, the signal-to-noise ratios of the spectra in each frame will differ depending on the observing conditions during the individual integrations, providing an additional test of how well our $v \sin (i)$ measurements and errors hold up under varying levels of signal-to-noise. To perform this test, we analyzed Hydra frames taken of two different fields, one observed during December 1997 and another in December 1998. In the former case, six individual 
integrations of the same field were taken over a period of about three weeks. In the latter case, five exposures of another field were obtained on a single night. To measure $v \sin (i)$ in the single frames, we used the identical method described in Section 2.4 used to measure velocities in the combined frames. Because the integration times for the single frames were only $1 / 5$ to $1 / 6$ of the total combined integration, many of the stars in the single frames had insufficient signal to measure $v \sin (i)$. Sixty-one objects did yield reliable multiple measurements, and of these, 34 have $v \sin (i)$ $>11 \mathrm{~km} \mathrm{~s}^{-1}$. For these 34 stars, we compared their calculated $v \sin (i)$ errors $( \pm v \sin (i) /(1+r))$ with the standard deviation of the individual $v \sin (i)$ values from the multiple measurements. For 31 of 34 stars $(91 \%)$, the $v \sin (i)$ error was equal to or larger than the standard deviation of the individual measurements, and in a handful of cases much larger. For the remaining three stars, the computed $v \sin (i)$ error equalled $\sim 60-80 \%$ of the standard deviation. Overall the test indicates again that our adoption of $\pm v \sin (i) /(1+r)$ as a reasonable estimate of the one-sigma error on the $v \sin (i)$ values is both valid and appropriate.

A possible source of systematic error in the $v \sin (i)$ measurement process comes from template mismatch, i.e., an error that may arise if an object spectrum is cross-correlated against a template star that does not exactly match the object's spectral type. Template mismatch might be a factor in our data set because we do not have a template star to match each individual spectral type of our objects, but instead have a set of five templates spanning the approximate range of our targets. To investigate the effect of template mismatch, a set of 40 stars was randomly chosen from the 238 for which $v \sin (i)$ was successfully measured. These 40 stars were cross-correlated against template stars hotter than and cooler than the correct template based on the star's spectral type. For example, a K6 target that was originally cross-correlated against the K7V template star Gliese 114 was for this test cross-correlated against Gliese $144(\mathrm{~K} 2 \mathrm{~V})$ and Gliese 15A (M1.5V). Comparisons were made of the $v \sin (i)$ values resulting from these tests for the 27 stars with $v \sin (i)$ values of at least $11 \mathrm{~km} \mathrm{~s}^{-1}$. In 22 of the 27 cases $(82 \%)$, the $v \sin (i)$ values measured using the hotter or cooler templates matched the original $v \sin (i)$ within the one-sigma error bars, and in all 27 cases they matched within less than three sigma. The mean difference between the original $v \sin (i)$ value and that measured using a hotter template was $-2.5 \mathrm{~km} \mathrm{~s}^{-1}$, with a standard deviation of $3.9 \mathrm{~km} \mathrm{~s}^{-1}$. The mean difference between the original value and the value when a cooler template was used was $+1.3 \mathrm{~km} \mathrm{~s}^{-1}$, with standard deviation $5.1 \mathrm{~km} \mathrm{~s}^{-1}$. In other words, using a template that is too hot for a given target star causes the $v \sin (i)$ measured to be too large, and the opposite is true for a template that is too cool. In the case of the too-cool template, the mean difference is not statistically significant, whereas it is marginally so for the templates that were too hot. We note however that the test performed mimics an extreme situation in which the stars have been cross-correlated against templates that are several spectral subclasses away from the appropriate spectral type. Even under such circumstances, the mean systematic difference for the group of 27 stars is very small. The overall agreement within the errors for individual stars indicates that the uncertainty associated with template mismatch is accounted for by our estimated errors on $v \sin (i)$.

Another potential source of error in our $v \sin (i)$ values is line blending in the stellar spectra. 
Including regions of the spectra that have strong line blending could bias our results toward larger derived $v \sin (i)$. To test for this effect, 40 stars were randomly chosen (a different set than used for the template mismatch test) from the 238 with measured $v \sin (i)$. Several regions with possible line blending were identified using a high signal-to-noise daytime sky spectrum. These regions were then excluded from the cross-correlation process. The resultant $v \sin (i)$ values for 28 stars for which $v \sin (i)$ was at least $11 \mathrm{~km} \mathrm{~s}^{-1}$ were compared with those originally measured. For 27 of the 28 stars $(96 \%)$, the $v \sin (i)$ values from the test were the same as the original values within two sigma, and in all 28 cases they agreed within less than three sigma. The mean difference between the original $v \sin (i)$ and that measured with line-blended regions excluded was $0.45 \mathrm{~km} \mathrm{~s}^{-1}$, with a standard deviation of $3.0 \mathrm{~km} \mathrm{~s}^{-1}$. This difference is not significant. Thus, as in the case of template mismatch, we again find that the potential systematic uncertainties associated with line blending are taken into account by the uncertainties we have estimated for our $v \sin (i)$ values.

Figure 7 shows a histogram of the heliocentric radial velocity values for the stars for which $v \sin (i)$ was measured. The outlier appearing at $-8 \mathrm{~km} \mathrm{~s}^{-1}$ is JW 50 , one of our SB2 candidates; to derive the radial velocity for this star, we (arbitrarily) chose to fit the blue peak in the crosscorrelation function, since the two peaks were well-separated. The star with a radial velocity of $87 \mathrm{~km} \mathrm{~s}^{-1}$ is JW 363, which has an ONC membership probability from Jones \& Walker (1988) of $98 \%$, so this star may be an SB1. The mean of the distribution, excluding JW 363 and all SB2 candidates, is $26.7 \mathrm{~km} \mathrm{~s}^{-1}$, with a standard deviation of $5.6 \mathrm{~km} \mathrm{~s}^{-1}$. This is comparable (although with larger errors) to the expected mean radial velocity for stars in this region, which is $\sim 25 \mathrm{~km} \mathrm{~s}^{-1}$ with a dispersion of $\sim 2 \mathrm{~km} \mathrm{~s}^{-1}$ (Jones \& Walker 1988; R. Mathieu, unpublished data).

Fourteen of the stars in our sample with $v \sin (i)>11 \mathrm{~km} \mathrm{~s}^{-1}$ have had their $v \sin (i)$ values measured in other studies. Figure 8 shows a comparison between our measured $v \sin (i)$ values and those from work by Duncan (1993) and Wolff, Strom, \& Hillenbrand (2001). In the figure, squares represent stars that our study has in common with Duncan's study, and triangles are stars we have in common with Wolff, Strom, \& Hillenbrand. Duncan estimated that his $v \sin (i)$ values have an accuracy of $10-15 \%$, so the squares are shown with horizontal error bars equal to $15 \%$ of $v \sin (i)$. The points in the figure scatter around the line marking equal $v \sin (i)$ values from our study and the others; our measured values are larger for nine of the stars, and smaller for the remaining five. The mean difference between the $v \sin (i)$ values is $0.5 \pm 2.0 \mathrm{~km} \mathrm{~s}^{-1}$, indicating reasonable agreement between our values and those from previous work. The figure does show, however, that the random errors of all three studies have probably been underestimated, since about half of the points do not intersect the equality line within the error bars. Nevertheless, there are no obvious systematic differences between our $v \sin (i)$ values and those measured by other groups.

\subsection{1. $v \sin (i)$ Distributions}

The uppermost plot in Figure 9 shows the $v \sin (i)$ measurement results from this study, in the form of a histogram of the $v \sin (i)$ distribution for all 238 stars for which a $v \sin (i)$ was measured 
(including upper limits). In this and the other two plots shown in Figure 9, the number of stars in each bin has been normalized by the total number of stars in the particular sample. Approximately $68 \%$ of the stars have $v \sin (i)$ less than $20 \mathrm{~km} \mathrm{~s}^{-1}$. The remaining $32 \%$ of $v \sin (i)$ values are distributed in a "tail" that extends from $20 \mathrm{~km} \mathrm{~s}^{-1}$ to $\sim 100 \mathrm{~km} \mathrm{~s}^{-1}$.

One of the first systematic studies of T Tauri star rotational velocities was done by Vogel \& Kuhi (1981), who observed 50 stars in the Taurus-Auriga and Orion complexes. The Vogel \& Kuhi sample was somewhat brighter than our sample, having $V$ magnitudes in the range 11-15, whereas our stars have $V \sim 12-19$. Their stars were generally more massive than ours, with masses larger than $\sim 0.5 \mathrm{M}_{\odot}$. Vogel \& Kuhi found that three-quarters of the stars they observed had velocities below their observational limit of $25-35 \mathrm{~km} \mathrm{~s}^{-1}$, and concluded that stars with masses $<1.5 \mathrm{M}_{\odot}$ generally have $v \sin (i)$ less than $25 \mathrm{~km} \mathrm{~s}^{-1}$. A 1986 study by Hartmann et al. of 50 stars in TaurusAuriga and Orion found similar results. They observed stars with $V$ magnitudes brighter than 13-14, thus comparable to the brightest stars in our sample. Hartmann et al. concluded that nearly all the stars in the Taurus-Auriga complex have $v \sin (i) \lesssim 20 \mathrm{~km} \mathrm{~s}^{-1}$. Although the large majority of our sample stars have $v \sin (i)<20 \mathrm{~km} \mathrm{~s}^{-1}, 25$ stars have $v \sin (i)>45 \mathrm{~km} \mathrm{~s}^{-1}$. Twenty of the 25 stars with large $v \sin (i)$ values have masses less than $1.2-\mathrm{M}_{\odot}$, and the star with the largest $v \sin (i)$ value is JW 526, a $0.47-\mathrm{M}_{\odot}$ star. Thus the finding of Vogel \& Kuhi that stars with mass $<1.5 \mathrm{M}_{\odot}$ have velocities less than $25 \mathrm{~km} \mathrm{~s}^{-1}$ is not true for our sample. This is consistent with photometric studies (e.g., Choi \& Herbst 1996, Stassun et al. 1999, Herbst et al. 2000, 2001) which show that a number of the low-mass stars in the ONC have short rotation periods (e.g., less than a day or two).

One of the objectives of this study was to test whether the rotation data gained from photometric studies of pre-main-sequence stars (i.e., studies in which periods are measured via photometric monitoring) are biased in some way, or are truly representative of the rotational properties of young stars. To address this issue, we compared the $v \sin (i)$ distributions of the Periodic sample (the sample of stars known to be periodic from the VVO monitoring study) and the Control sample (the sample of stars selected to have luminosities and effective temperatures similar to the Periodic sample stars). (As noted in Section 2.1, about 30\% of the Control sample stars have since been found to be periodic.) This comparison is shown in the two lower plots in Figure 9, which show the $v \sin (i)$ distributions for 111 stars in the Periodic sample and 127 stars in the Control sample. Both distributions look similar to that of the full sample. One might expect that studies based on rotation periods could be skewed toward including a higher fraction of rapid rotators than appears in the overall population of young stars, because rapidly-rotating stars with more active dynamos might tend to have more prominent and/or persistent surface spots. In that case, the Periodic sample should have a larger proportion of rapid rotators compared to the Control sample. This may at first glance appear to be true, since there is a slight overabundance of rapid rotators in the Periodic sample distribution. (23\% of stars in the Periodic sample have $v \sin (i) \geq 30 \mathrm{~km} \mathrm{~s}^{-1}$, compared to $17 \%$ of the Control sample stars.) However, a Kolmogorov-Smirnov (K-S) test comparing the two samples suggests that the difference is not significant; the test gives a probability of 0.74 , indicat- 
ing that the $v \sin (i)$ distributions of the Periodic and Control samples do not differ. Therefore the idea that studies of pre-main-sequence rotation are biased by the use of photometrically-measured rotation periods is not borne out by our data.

$\mathrm{T}$ Tauri stars of similar mass and age are observed to have a wide range of rotation periods and, in some cases, a bimodal period distribution (Stassun et al. 1999; Herbst et al. 2000). A proposed explanation for the bimodality involves the concept of "disk-locking", in which a magnetic interaction between the disks around $\mathrm{T}$ Tauri stars and the stars themselves regulates their rotation rates (Königl 1991; Shu et al. 1994; Ostriker \& Shu 1995). In this picture, kilogauss-strength magnetic fields intercept the circumstellar disk, channel accretion flow onto the star's surface, and "lock" the star to the disk. The rotation rate of the star is then forced to be the same as that of the disk at the locking radius. The specifics of disk-locking, e.g., how long it lasts, why some stars may be locked to their disks while others are not, and exactly what role it plays in a star's angular momentum evolution, are unclear. Moreover, the observational evidence for disk-locking occurring during the T Tauri phase has recently been under debate (Stassun et al. 1999; Herbst et al. 2000).

In the disk-locking picture, a star that is rotating relatively slowly is thought to be locked to its circumstellar disk (or only recently unlocked), whereas a fast rotator either was never disklocked or has had time to spin up since it became unlocked. As a test of this idea, another useful comparison to make would be to compare the $v \sin (i)$ distributions of stars with disks and without them. To construct a sample of stars likely to have disks, we chose objects from our sample with characteristics like those of Classical T Tauri Stars (CTTS). CTTS often have strong emission lines and are thought to be experiencing accretion onto their surfaces from a circumstellar disk, resulting in surface hot spots that cause irregular light variations and flaring (Bertout 1989). Weak T Tauri Stars (WTTS), on the other hand, often have low-amplitude ( $\leq 0.5 \mathrm{mag})$, periodic light variations, possibly due to the presence of large cool spots on their surfaces, and lack obvious signatures of an accretion disk (Herbst et al. 1994).

We used the observational diagnostics from Hillenbrand et al. (1998) to choose a sample of possible CTTS and WTTS. These diagnostics, namely the near-infrared excess $\Delta\left(I_{\mathrm{C}}-K\right)$ and Ca II emission line strength $W_{\lambda}(\mathrm{Ca}$ II), are determined by Hillenbrand et al. and applied to stars in the ONC to investigate the frequency of circumstellar disks in the cluster. Here we adopt a combination of the criteria used by Hillenbrand et al. The disked (CTTS) sample we chose consists of stars that have no measured period, and have either (1) $\Delta\left(I_{\mathrm{C}}-K\right)>0.3$ and Ca II emission, or (2) $\Delta\left(I_{\mathrm{C}}-K\right)>0.5$. The non-disked (WTTS) sample is comprised of stars with measured periods, and either $(1) \Delta\left(I_{\mathrm{C}}-K\right)<0.1$ and $W_{\lambda}(\mathrm{Ca}$ II $)>0$, or $(2) \Delta\left(I_{\mathrm{C}}-K\right)<0$. We tried to use fairly stringent criteria while still choosing a good-sized sample of objects for each category, but the WTTS and CTTS samples are nevertheless small in size, with 30 and 36 objects, respectively. The $v \sin (i)$ distributions for the WTTS and CTTS samples are shown in Figure 10. Again, the numbers in each bin are normalized by the number of stars in the sample. The distributions appear to differ in the predicted sense; that is, the CTTS sample (stars likely to have disks) does contain a slightly larger fraction of slow rotators compared to the WTTS sample. A K-S test comparing 
the two distributions, however, yields a probability of 0.26 , meaning that any difference between them is not statistically significant.

\subsubsection{Comparing v $\sin (i)$ to Velocity Estimated from Rotation Period}

Because we know the rotation periods of most of the stars for which we measured $v \sin (i)$, we can compare the equatorial rotation velocities (calculated using the periods and radii estimated from the luminosities and effective temperatures) for those stars with their measured $v \sin (i)$ values. This comparison is shown in Figure 11: $v \sin (i)$ values for 153 stars are plotted versus equatorial velocities derived from the equation $v_{e q}=2 \pi R / P$. (Note that one star $-\mathrm{JW} 834-$ has a $v \sin (i)$ and period, but has neither a luminosity nor an effective temperature determination, so its radius cannot be calculated.) SB2 candidates are shown with open circles. For stars with $v \sin (i)$ less than or equal to our estimated limit of $11 \mathrm{~km} \mathrm{~s}^{-1}$, upper limit symbols are plotted. The solid line in the figure marks $v \sin (i)=v_{e q}$, and the dotted line marks $v \sin (i)=(\pi / 4) v_{e q}$. Assuming randomlyoriented stellar axes, the observed mean value of $\sin (i)$ should be $\pi / 4$, or 0.785 (Chandrasekhar \& Münch 1950). Therefore, at least in theory, the points are expected to scatter about the dotted line.

Figure 11 shows first of all that there is a strong correlation between $v \sin (i)$ and $v_{e q}$, thus demonstrating conclusively that the periodicity of $\mathrm{T}$ Tauri stars is caused by the rotation of stars with spotted surfaces. The correlation of these quantities is strong: results from two different non-parametric tests indicate that the probability that there is no correlation is between $10^{-24}$ and $10^{-27}$. In addition, the $v \sin (i)=v_{e q}$ limit is respected by nearly all the stars to within the errors of the measurement. The one notable exception, JW 275, is discussed below. This is a gratifying result and an important confirmation of the basic assumptions underlying pre-mainsequence rotation studies to date.

A noticeable feature of Figure 11 is that the majority of points fall below the $v \sin (i)=$ $(\pi / 4) v_{e q}$ line, indicating that the average $\sin (i)$ value we observe is lower than the expected value. To investigate the significance of this deviation, we begin by calculating a mean $\sin (i)$ including all 153 stars with both $v \sin (i)$ and $v_{e q}$ values. This yields $\langle\sin (i)\rangle=0.82 \pm 0.09$, with a standard deviation of 1.1. This result is dominated by a few very high values of $\sin (i)$ that cannot simply be understood in terms of random errors. The most extreme example is JW 275, which has a radius of $2.3 \mathrm{R}_{\odot}$ and VVO period of 20.1 days, implying an equatorial velocity of $5.9 \mathrm{~km} \mathrm{~s}^{-1}$. Its measured $v \sin (i)$, however, is $78 \mathrm{~km} \mathrm{~s}^{-1}$, completely inconsistent with expectation. This star represents a clear example of a gross error; either its rotation period or its $v \sin (i)$ is incorrect. The star's period was measured from only a single longitude, and therefore could be a beat period. If the measured 20.1-day period is actually the beat period between a 1-day sampling interval and a 1.05-day rotation period, then $v_{e q}$ for the star should be $112 \mathrm{~km} \mathrm{~s}^{-1}$. Combining this with the measured $v \sin (i)$ yields the quite reasonable $\sin (i)$ value of 0.69 . Rotation periods close to one day are difficult to distinguish from beat periods in data obtained at a single longitude. Clearly 
this star and others like it need to be removed from the sample before drawing inferences about the observed distribution of $\sin (i)$. Stars whose $v \sin (i)$ measurements are too small compared to their likely errors should also be removed before $\langle\sin (i)\rangle$ is calculated.

Accordingly, we first restrict the sample to those stars with $v \sin (i)$ greater than $11 \mathrm{~km} \mathrm{~s}^{-1}$, our approximate measurement limit based on the discussion in Section 2.4. We also remove from the sample the remaining stars (which include JW 275) with predicted $v_{e q}$ values less than $11 \mathrm{~km} \mathrm{~s}^{-1}$. The rationale for eliminating those stars is first of all that if the period and radius are accurate and $v_{e q}$ is less than $\sim 11 \mathrm{~km} \mathrm{~s}^{-1}$, we do not expect to measure a reliable $v \sin (i)$ given our resolution. Alternatively, if the period and radius are in error, the star should for that reason be culled from the sample. For the latter reason we exclude three stars with $\sin (i)>1$ that may be cases similar to JW 275. Applying these conditions leaves a sample of 86 stars with precise $\sin (i)$ estimates. This sample yields $\langle\sin (i)\rangle=0.64 \pm 0.02$ with standard deviation $=0.23$. (If we include the abovementioned three stars with $\sin (i)>1$, the result is the same within the errors: $\langle\sin (i)\rangle=0.67 \pm 0.03$, standard deviation 0.26.) Furthermore, if we construct an even more robust sample of stars with $v \sin (i)$ and $v_{e q}$ greater than $13.6 \mathrm{~km} \mathrm{~s}^{-1}$, our estimate of the formal spectral resolution, then $\langle\sin (i)\rangle=0.59 \pm 0.025$ (standard deviation $=0.21$ ). The average inclination for this sample of 68 objects is $38 \pm 2$ degrees. ${ }^{4}$

Results similar to ours have been reported in previous studies that combined $v \sin (i)$ data with rotation periods of pre-main-sequence stars. Hartmann et al. (1986) compared $v \sin (i)$ and $v_{e q}$ for six T Tauri stars and found that the $v \sin (i)$ values of two of the slower rotators in their sample fell below the expected $v_{e q}$ values. Weaver (1987) combined data from Hartmann et al. (1986) with that from other studies to produce a sample of 20 stars for which both $v \sin (i)$ and period were known. Weaver found that the mean inclination for the sample of 19 stars with $\sin (i)<1$ was 40 degrees rather than the expected value of 57 degrees. The average $\sin (i)$ value from Weaver's data is 0.63. More recently, Soderblom et al. (1999) observed 35 stars in the open cluster NGC 2264. For the $\sim 20$ stars in their sample with measured $v \sin (i)$ and period and $\sin (i) \leq 1$, the average $\sin (i)$ value was 0.6 , also in agreement with our result.

There are a number of possible explanations for the discrepancy between observed average $\sin (i)$ values and theoretical expectation. To clarify the possible reasons, we express $\sin (i)$ in terms of quantities either observed directly or derived from observations by writing:

$$
\sin (i)=(\text { constant })(v \sin (i)) P T_{\mathrm{eff}}^{2} L^{-1 / 2}
$$

Systematic errors in $v \sin (i)$, luminosity, effective temperature, and/or rotation period could give rise to the observed deviation from $\langle\sin (i)\rangle$. The approximate size of the systematic error(s) would

\footnotetext{
${ }^{4}$ We note that the errors on the mean values given here were calculated assuming a Gaussian distribution. This is not strictly valid as $\sin (i)$ is not expected to be normally-distributed. Our actual observed $\sin (i)$ values, however, do follow a smooth, fairly symmetric distribution with a prominent peak. Thus we have used the mean and its standard error to characterize the observed distribution.
} 
be $0.2 / 0.6$, or about $30 \%$. Alternatively, the smaller-than-expected $\langle\sin (i)\rangle$ might be due to a real physical phenomenon. We have explored each of these possibilities and address them below.

Physical causes. - There may be an actual physical cause behind the lower average $\sin (i)$ we observe. For example, Weaver (1987) suggested that it could be the result of selection against stars with high axial inclination in rotation studies, because circumstellar material obscures the stellar photosphere. We have explored this idea and find from numerical simulations that in order to reduce the average value of $\sin (i)$ from 0.79 to 0.60 , all stars with inclinations between 52 and 90 degrees must be systematically excluded from period studies. Given a random distribution of rotation axes, this means about $60 \%$ of the stars would have to be excluded, which seems unlikely. Alternatively, it is possible that rotation axes in young clusters like the ONC and NGC 2264 are aligned rather than oriented randomly. Some have suggested that the formation of a star cluster from a rotating molecular cloud threaded by a uniform magnetic field should eventually lead to alignment of stellar rotation axes (see Bodenheimer 1995 and references therein). Evidence with regard to such alignment is mixed, with some data showing alignment of structures within molecular clouds (see Heiles et al. 1993 and included references) and some indicating that stellar rotation axes are randomly oriented in certain clusters (e.g., the Pleiades; Stauffer 1991). Another explanation could be that the prominent surface spots that give rise to T Tauri stars' photometric variability are more likely to appear near the poles of the stellar rotation axis than near the equator. Consequently the stars that are detected in photometric period studies (and thus appear in Figure 11) would tend to have more pole-on inclinations, and thus have $\sin (i)$ values that are truly lower than expected from a random distribution. On the other hand, if a star were seen exactly pole-on, there would be no photometric variation observed. In any case, Figure 9 shows that there is not a significant bias between the $v \sin (i)$ distributions of stars selected from period studies (the Periodic sample) compared to those selected via only their luminosities and effective temperatures (the Control sample).

Systematic errors in $v \sin (i)$.- - One way to produce a smaller-than-expected average $\sin (i)$ value is if our measured $v \sin (i)$ values are systematically low. Increasing our values by at least $30 \%$ would bring $\langle\sin (i)\rangle$ close to its expected value. The comparison of our $v \sin (i)$ values for the 14 stars we have in common with other studies (see Figure 8) shows, however, that for over half the stars, our measured $v \sin (i)$ values are actually slightly larger than the values from other work. The overall mean difference between our $v \sin (i)$ values and those of the two other studies is $0.5 \pm 2.0 \mathrm{~km} \mathrm{~s}^{-1}$. This, coupled with the fact that other independent studies of pre-main-sequence stars in NGC 2264, Taurus and Orion also found average $\sin (i)$ values of approximately 0.6 , makes it seem unlikely that a systematic $v \sin (i)$ error is wholly responsible for the $\langle\sin (i)\rangle$ discrepancy. On the other hand, it is difficult to rule out the possibility that some smaller systematic error in $v \sin (i)$ is contributing to the problem. In Section 3.1 we explored systematic uncertainties associated with line blending and possible mismatches in spectral type between the targets and cross-correlation templates. In those cases the associated uncertainties were accounted for by our estimated errors. But there could be other systematic effects at work. For example, narrow-lined spectra from dwarf 
stars are used as cross-correlation templates against which the pre-main-sequence stellar spectra are compared. It is feasible that a difference in the limb darkening law between dwarfs and premain-sequence stars could have an effect on the $v \sin (i)$ calibration, introducing a systematic error. Such a systematic error could potentially affect the other $v \sin (i)$ studies mentioned, most of which also used dwarfs as templates for the cross-correlation.

Errors in the photometric periods. - Still another explanation for the lower average sin $(i)$ value could be that the rotation periods inferred from photometric studies are, in some cases, not the actual rotation periods of the stars. Instead, they could be false detections, harmonics of the rotation period, beat periods between an observing interval (normally one day) and the rotation period, or, in the case of spectroscopic binaries, related instead to the orbital period of the system. We consider each of these ideas in turn.

It is unlikely that many of the photometric periods are false detections rather than true periods, since statistical tests are used to eliminate these and, in many individual cases of low $\sin (i)$ values, there are confirming period determinations at different epochs (see Herbst et al. 2000). Moreover, the presence of false detections should produce a random scatter in the $v \sin (i)-v_{e q}$ plane, tending to obscure the observed correlation rather than skew it towards small values of $\sin (i)$. Harmonics, on the other hand, could explain at least some of what is displayed in Figure 11. T Tauri stars such as V410 Tau (Herbst 1989) and some stars in the ONC (Stassun et al. 1999; Herbst et al. 2001) have displayed so-called "period doubling", i.e., the period determined at one epoch is later shown to be half of the true rotation period because the star originally had significant spots on two opposing hemispheres. The effect of correcting a period-doubling error would be to decrease $v_{e q}$ by a factor of two for any such star. Taking the 68-star sample described above and arbitrarily doubling the period of every star with an inferred $\sin (i)<0.60$ (34 of the 68 stars) increases $\langle\sin (i)\rangle$ to the expected value. Whether this is part or all of the problem can only be determined by continued careful monitoring of ONC stars to see whether period doubling occurs for a significant number of them. Comparisons made so far indicate that this is not a major contributor to the problem (Herbst et al. 2001; Carpenter, Hillenbrand \& Skrutskie 2001).

Another explanation for those stars with larger-than-expected $v_{e q}$ could be that the observed photometric periods are actually beat periods. This would preferentially affect stars with shorter periods (i.e., $\sim 1$ day or so) since the periods are closer to the observing frequency. Both the VVO (Herbst et al. 2000) and ESO (Herbst et al. 2001) data sets were obtained at single longitudes (although with multiple observations per night) and are thus more susceptible to the beat phenomenon than the Stassun et al. (1999) study, which combined data from widely-separated longitudes. Herbst et al. (2001) compared their data with Stassun et al. (1999) and found that of 111 stars monitored by both studies, 98 yielded identical periods, two were likely examples of period doubling, one appeared to be a false detection, and the other 10 were related as beat periods with a 1-day sampling interval. This suggests that about $10 \%$ of the sample of periodic stars might be contaminated by beat periods or harmonics. Again, the continued monitoring of ONC stars, preferably from observatories well separated in longitude, is necessary in order to determine 
whether these phenomena are important in the interpretation of Figure 11.

Lastly, it is possible that some of the periodicity detected in photometric studies is of orbital origin. Close spectroscopic binaries are variable for a number of reasons including eclipses, tidal distortion and the reflection effect. Such stars may not be rotationally locked and observed periods could be the orbital period or a related harmonic. Alternatively, in the case of tidal locking, both tidal distortion and reflection can lead to period doubling. As mentioned in Section 3.1, seven stars show evidence indicating that they might be spectroscopic binaries. Five of these stars have measured periods and are marked with open circles in Figure 11. Of the five stars with the largest $v_{e q}$ values in the figure, two are SB2 candidates. If these stars' observed periods arise from orbital motion rather than rotation, then they should not be plotted on Figure 11.

In summary, despite the fact that the rotation periods are more precise than the other quantities contributing to $\sin (i)$ (typically being known to $\sim 1 \%$ or better; see, e.g., Choi \& Herbst 1996), they are prone to gross errors such as aliasing and period doubling. These effects probably account for the small number of outliers removed from the sample used to calculate our observed $\langle\sin (i)\rangle$. It is difficult to see how they could account for the remaining systematic effect, which is accompanied by a relatively small scatter in $\sin (i)$. In order for such period errors to account for the remaining systematic effect, a significant number of rotation periods would need to have been underestimated. This could be the case if period-doubling plays a major role. This seems unlikely, however, in light of the general agreement found between periods measured by different observers at different epochs with different sampling intervals (e.g., Stassun et al. 1999, Herbst et al. 2001, Rebull 2001, Carpenter et al. 2001).

Overestimated luminosities.- Equation (1) shows that $\sin (i)$ depends on $L^{-1 / 2}$; as a result, systematically overestimated luminosities for our target stars could give rise to a lower-thanexpected $\langle\sin (i)\rangle$. For the sample of 68 stars, the true stellar luminosities would have to be approximately $60 \%$ of the current measured values in order to yield an average $\sin (i)$ consistent with the expected value. Weaver (1987) has suggested that the observed luminosities of pre-mainsequence stars might be artificially inflated due to the presence of a circumstellar disk. This idea could explain why the $\sin (i)$ discrepancy apparent in our data has been seen by others (namely Soderblom et al. 1999 and Weaver 1987), who have observed pre-main-sequence stars in different clusters with different observational methods. On the other hand, this requires that the large majority of our target stars have a relatively luminous disk, i.e., one that is responsible for $\sim 40 \%$ of the total optical luminosity of the disk-star system. Hillenbrand et al. (1998) estimate the disk fraction in the ONC to be between $55 \%$ and $90 \%$, so the majority of ONC stars may indeed have disks. However when we examine the $\Delta\left(I_{\mathrm{C}}-K\right)$ values of the 68 -star sample, we find that only about $40 \%$ have $\Delta\left(I_{\mathrm{C}}-K\right)>0.3$, indicating the presence of a prominent disk. There are uncertainties associated with determining $\Delta\left(I_{\mathrm{C}}-K\right)$, and Hillenbrand et al. note that the errors (which are expected to be primarily negative, reducing $\Delta\left(I_{\mathrm{C}}-K\right)$ from its true value) may be as large as 0.1-0.3 magnitudes for stars with spectral types K2 to M3. Nevertheless it seems unlikely that the large majority of our stars have disks that are sufficiently luminous to account for the observed 
$\langle\sin (i)\rangle$ discrepancy.

Another uncertainty associated with the luminosities is the extinction correction used to derive them. Systematically overestimated extinction values would result in too-large luminosities. Our luminosities come from Hillenbrand (1997), who suggests that if anything the luminosity values may be underestimated, if there is unaccounted-for accretion activity or a nonstandard or spatiallyvariable extinction law in the ONC. In calculating the overall uncertainty on the luminosities, Hillenbrand assumes an error in $A_{V}$ of 0.5 magnitudes, which is larger than expected and which corresponds to an uncertainty of 0.12 in $\log \left(L_{*} / L_{\odot}\right)$. Other sources of error that may contribute are intrinsic uncertainties due to photometric variability, errors from translating spectral type and color to luminosity, and uncertainties in the stellar distances. Hillenbrand estimates that these contribute uncertainties of $0.12,0.15$ and 0.075 dex, respectively, for a total combined uncertainty of less than 0.2 dex, on average. Subtracting this amount from the luminosities for the sample of 68 stars yields $\langle\sin (i)\rangle=0.75 \pm 0.03$, which is close to the expected value. But again, Hillenbrand suggests that if a systematic error is present in the luminosities, it would likely go the other way, leading to underestimated values instead of too-large ones.

A detailed discussion of the observational uncertainties associated with estimates of stellar luminosities is given in Hartmann (2001), who explores the effect of such errors on age estimates in star-forming regions. In addition to discussing each of the sources of error mentioned above, Hartmann suggests that unresolved binary companions can have a potentially large effect on luminosity determinations. Moreover, this would be a systematic effect: the presence of unidentified companion stars around our targets would bias their luminosities to higher values. Hartmann models the effect of unresolved binaries on luminosities and estimates that the shift in $\log L$ would be $\sim 0.2$, under the assumption that nearly all stars have unidentified companions. As explained above, a shift of this magnitude could bring $\langle\sin (i)\rangle$ close to its expectation value, so this idea could potentially help explain the observed discrepancy.

Underestimated effective temperatures. Because $\sin (i) \propto T_{\text {eff }}^{2}$, the $\sin (i)$ values we derive are much more sensitive to errors in the effective temperatures than to errors in the luminosities. The effective temperatures we use come from Hillenbrand (1997), who determined spectral types for ONC stars and then converted them to $T_{\text {eff }}$ values using the calibration of Cohen \& Kuhi (1979), with a few modifications. We find that increasing $T_{\text {eff }}$ for our sample stars by 400 to 600 Kelvin (without changing the luminosity) yields good agreement between the measured and expected $\sin (i)$ distributions. Figure 12 shows the effect of a $600-\mathrm{K}$ increase in temperature in the $v \sin (i)$ versus $v_{e q}$ plane. All 153 stars that have $v \sin (i)$ and $v_{e q}$ measures are shown. The points now scatter around the dotted line representing $v \sin (i)=(\pi / 4) v_{e q}$. The figure demonstrates that increasing the temperatures can rectify the $\langle\sin (i)\rangle$ discrepancy; however, this fix of the problem requires that the temperatures have been significantly underestimated.

An examination of the literature suggests that it is possible that uncertainty inherent in the conversion from spectral type to $T_{\text {eff }}$ could cause the temperatures to be off by the required amount. 
Padgett (1996) and King (1998), for example, discuss problems with the existing spectral type- $T_{\text {eff }}$ calibration for pre-main-sequence stars. These include the uncertainty with regard to whether a dwarf or giant temperature scale is more appropriate, as discussed by Hillenbrand (1997) and White et al. (1999). King estimates that the size of the errors in $T_{\text {eff }}$ determinations for pre-mainsequence stars is 500-700 K. Padgett finds differences as large as $960 \mathrm{~K}$ between $T_{\text {eff }}$ values derived from line ratio measurements in high resolution spectra and those obtained from spectral type$T_{\text {eff }}$ calibration. She finds that temperatures derived from metallic line ratios are generally higher than those determined from lower-resolution spectral classification. The assumption that pre-mainsequence stellar atmospheres can be precisely calibrated by comparison with the atmospheres of dwarfs or evolved stars is likely to break down at some point. A major difference is that premain-sequence stars have spots - indicating the presence of strong magnetic fields - covering a large portion of their surfaces. The effect of these spots on the spectral type- $T_{\text {eff }}$ calibration is not well-understood. Moreover, Cohen \& Kuhi (1979) note that it is unclear exactly what the physical meaning of $T_{\text {eff }}$ is in a star with different emitting regions (such as the photosphere, chromosphere, extended atmosphere, and circumstellar disk) that each contribute significant amounts of flux.

To summarize, we find a significant departure of the distribution of measured $\sin (i)$ values from what is expected for a random distribution of observed inclination angles. This departure has also been seen in previous studies of pre-main-sequence stars. It could have a number of explanations, including real physical conditions, selection effects, and/or systematic errors in one or more of the quantities that go into calculating $\sin (i)$. If this were entirely due to an error in the spectral type-to$T_{\text {eff }}$ calibration, an adjustment by 400 to 600 Kelvin towards hotter temperatures than determined by Hillenbrand (1997) is required to solve the problem. This is large, but perhaps not impossible, given the current state of our understanding of effective temperatures of pre-main-sequence stars. If the $T_{\text {eff }}$ values of the stars have been underestimated, the masses and ages (determined by plotting the stars on an H-R diagram and interpolating the masses and ages from evolutionary model tracks) also need to be adjusted. (For example, a $0.25-\mathrm{M}_{\odot}$ star becomes a $0.4-\mathrm{M}_{\odot}$ star if its temperature is increased by $600 \mathrm{~K}$.) This in turn would have important implications for conclusions drawn about pre-main-sequence stellar evolution in the ONC and other clusters and associations.

\subsection{Stellar Radii}

A main goal of this study is to combine the $v \sin (i)$ measurements we obtain with rotation period measurements from other studies in order to constrain the radii of the stars in our sample. Because $P v \sin (i)=2 \pi R \sin (i)$, the $v \sin (i)$ and period of a star can be used to calculate its minimum radius, $R \sin (i)$. With enough data, the rotational velocities and periods can provide statistical estimates of radii (without the $\sin (i)$ ambiguity) for stars grouped by their similar characteristics. Direct measurements of the radii of pre-main-sequence stars are hard to come by, since so far only four pre-main-sequence eclipsing binaries have been discovered (Popper 1987; Casey et al. 1995, 1998; Andersen 1991; Mamajek et al. 2000; Covino et al. 2000). Therefore 
other methods for independently estimating stellar radii can be valuable tests of the reliability of the radii of pre-main-sequence stars and hence the accuracy with which they can be placed on the theoretical H-R diagram.

We have combined period and $v \sin (i)$ measurements to calculate minimum radii for individual stars with $v \sin (i)$ greater than our estimated limit of $11 \mathrm{~km} \mathrm{~s}^{-1}$. It should be emphasized that these minimum radii are not subject to the uncertainties in luminosity and effective temperature discussed in the previous section, but instead rely only on the measured period and $v \sin (i)$. There are 101 such stars not including JW 275, which has a period that is likely in error, as noted in Section 3.1.2. (Calculating a minimum radius using JW 275's measured period of 20.1 days yields an $R \sin (i)$ of $30.8 \mathrm{R}_{\odot}$; if its true period is instead 1.05 days, its $R \sin (i)$ is $1.61 \mathrm{R}_{\odot}$.) Minimum radii for the 101 stars are given in Table 1 in units of the solar radius, and Figure 13 shows a histogram of the values. They range from less than one to more than five solar radii, with the majority $(80 \%)$ having $R \sin (i)<2.5 \mathrm{R}_{\odot}$. The median of the distribution is $1.7 \mathrm{R}_{\odot}$.

Next we turn to making statistical estimates of the radii of groups of stars located in the same part of the H-R diagram. This calculation requires a sample of stars with reliable $v \sin (i)$ and period measurements, as well as known $L$ and $T_{\text {eff }}$ values. $L$ and $T_{\text {eff }}$ are needed only so that the stars can be grouped in a way that will make the radii derivation meaningful; again, the radii we calculate are completely independent of these two quantities. The same potential sources of error that were relevant when we explored the magnitude of the $\langle\sin (i)\rangle$ discrepancy in Section 3.1.2 must be considered here as well. For example, stars with $\sin (i)$ values much larger than 1 may have rotation periods that are in error. In addition, $v \sin (i)$ values near our estimated measurement limit $\left(11 \mathrm{~km} \mathrm{~s}^{-1}\right)$ may be less certain than larger values. To derive the most robust average radii possible with these data, we use the 68-star sample - consisting of stars with accurate periods and $v \sin (i) \geq 13.6 \mathrm{~km} \mathrm{~s}^{-1}$ — that was constructed to help characterize the $\langle\sin (i)\rangle$ problem.

We want to calculate average radii for groups of stars that are in approximately the same evolutionary stage. Consequently the stars must be binned according to their location in the theoretical H-R diagram. Even if there is a systematic error in $T_{\text {eff }}$ such as that discussed in Section 3.1.2, the relative locations of the sample stars should be fairly constant, so that grouping them by effective temperature should still be reasonable. Using as a guide the way in which the stars are arranged on the H-R diagram, a series of equal-sized bins was chosen that would maximize the number of stars within each bin (and the number of stars that could be binned) while still retaining adequate resolution to derive meaningful average radii. The bins are shown as solid lines in Figure 14. They each cover 0.048 units in $\log T_{\text {eff }}$ space and 0.37 units in $\log \left(L / L_{\odot}\right)$ space. The 68 sample stars used here are shown as filled circles; three of them fall outside of the chosen bins. The dotted lines marked on the figure are lines of constant radius, at intervals of $1 \mathrm{R}_{\odot}$, beginning at the bottom with $1 \mathrm{R}_{\odot}$.

Eleven of the 30 bins contain at least two stars. For these bins, an average stellar radius was calculated, by first computing $R \sin (i)$ for each individual star using the measured $v \sin (i)$ 
and period, averaging the projected radii for the stars in the bin, and finally dividing the average $R \sin (i)$ by the average expected value of $\sin (i), \pi / 4$. The radii are given in Table 3 . The first three columns of the table specify the bin number and luminosity and effective temperature range spanned by the bin. Column 4 gives the number of stars in the bin. Column 5 gives the mean $R \sin (i)$ value and its error, and column 6 lists the mean radius and error. The last column lists, for comparison, the average radius corresponding to the center of each $L$ and $T_{\text {eff }}$ bin. The mean difference between the radii calculated from $v \sin (i)$ and $P$ and the radii for the centers of the bins is -0.70 , with a standard deviation of 0.74 . Some difference between the $R$ values calculated using $v \sin (i)$ and period and those based on the locations of the stars in the H-R diagram is of course expected, in light of the data shown in the $v \sin (i)$ versus $v_{e q}$ plot and the accompanying discussion.

The calculation of $\langle R\rangle$ from period and $v \sin (i)$ yields a measurement of the radii of our sample stars that is independent of the usual methods for deriving stellar radii, and provides a snapshot of the evolution of low-mass stars as they contract toward the main sequence. To investigate whether we can actually observe that stars are contracting (i.e., use our data to show this geometrically), we have grouped the bins in Table 3 according to their $T_{\text {eff }}$ values, then ordered bins with the same $T_{\text {eff }}$ values by decreasing luminosity. Figure 2 shows that the evolutionary tracks in the portion of the H-R diagram in which our stars are located run fairly vertically. Therefore, examining the average $R \sin (i)$ values in sets of vertical bins (i.e., comparing bin A1 to A2, B1 to B2, etc.) should tell us whether stars of similar mass are actually contracting as they approach the Zero Age Main Sequence (ZAMS).

Examination of Table 3 shows that the mean radii do decrease with decreasing luminosity for bins in the same temperature range. However, the relative errors on $\langle R \sin (i)\rangle$ are as large as $20-30 \%$ in some cases. As a result, there is evidence for contraction at greater than the three-sigma level in only one pair of bins, B2 to B4. This is the only instance in which non-adjacent bins can be compared to each other. So although the mean values do decrease, we cannot state with absolute certainty that we are observing pre-main-sequence contraction for stars within a given temperature range. If we could include more stars with reliable $v \sin (i)$ and period in each bin (and by doing so reduce the errors on $\langle R \sin (i)\rangle)$ or could compare bins that span a larger range in radius, we might find more definitive evidence for contraction of stars in a specific temperature range.

We can look for overall evidence of contraction for the entire sample of 68 stars by combining the information in the five sets of vertical bins shown in Table 3. For each set of vertical bins (A, B, $\mathrm{C}, \mathrm{D}$, and $\mathrm{E}$ ) listed in the table, we can calculate the relative decrease in $\langle R \sin (i)\rangle$ with decreasing luminosity. For example, the relative change in radius $(\Delta R \sin (i) / R \sin (i))$ for bins A1 to A2 is 0.21 . Calculating this quantity for each of the five sets of vertical bins and averaging the result yields a mean relative change in projected radius of $0.49 \pm 0.16$. This result is positive - i.e., shows that stars in our sample are contracting as they approach the main sequence - at the three-sigma level. This is the first time that pre-main-sequence contraction has been verified geometrically.

To conclude, these data show marginally significant evidence supporting a real spread in radius 
(and therefore age) at a given mass in the ONC. It is clear that to proceed further (for example, determining an accurate age spread for the cluster) requires a larger sample of stars with accurate period and $v \sin (i)$ measurements. However, the promise of this method has now been clearly demonstrated.

\section{Summary \& Conclusions}

We have obtained high-dispersion spectroscopic data for 256 pre-main-sequence stars in the Orion Nebula Cluster, with the aim of measuring their $v \sin (i)$ values. Our intention was to combine the data with that from previous studies of the ONC that measured rotation periods via photometric monitoring, in order to better understand the rotational evolution of $\mathrm{T}$ Tauri stars and to arrive at statistical estimates of the sizes of these stars. A set of 118 objects with known periods (the Periodic sample) was chosen along with 138 stars from the same portion of the H-R diagram whose periods were not known (the Control sample). Most of the targets are low-mass ONC members with spectral types from early-K to mid-M. Spectra with sufficient signal-to-noise to measure $v \sin (i)$ were obtained for 238 of the 256 targets. Our results are as follows:

1. Approximately two-thirds of the 238 sample stars with good-quality spectra have $v \sin (i)$ less than $20 \mathrm{~km} \mathrm{~s}^{-1}$, and the $v \sin (i)$ values of the remaining stars are distributed in a tail extending to $\sim 100 \mathrm{~km} \mathrm{~s}^{-1}$.

2. We have compared the $v \sin (i)$ distributions of the Periodic and Control samples in order to determine whether photometric rotation period studies are truly representative of the rotational properties of pre-main-sequence stars. The differences between the distributions are not statistically significant. The same is true when we compare a sample of stars likely to be accreting material from their circumstellar disks (CTTS), and perhaps "disk-locked", to a sample that does not show evidence for accretion (WTTS).

3. We find a strong correlation between $v \sin (i)$ and equatorial rotation velocity $v_{e q}$ derived by combining the stars' periods with radii calculated from their published $L$ and $T_{\text {eff }}$ values. This shows definitively that the observed periodicity of T Tauri stars is, in the majority of cases, caused by the rotation of spots on their surfaces.

4. The average $\sin (i)$ value for our stars, calculated from $\sin (i)=v \sin (i) / v_{e q}$, is significantly lower than the mean value expected for a random distribution of stellar rotation axes. This result, which has been seen in previous studies with smaller samples, could be due to systematic errors in one or more of the observed quantities that go into the $\sin (i)$ calculation (namely $v \sin (i)$, period, $L$, and $T_{\text {eff }}$ ), or to a real physical phenomenon. We explore these possibilities and find that the correct $\sin (i)$ value is produced if we assume that the effective temperatures of our program stars have been underestimated by 400-600 Kelvin.

5. We have calculated minimum radii for stars that have rotation period measurements and $v \sin (i)$ 
greater than our estimated measurement limit. The $R \sin (i)$ values range from 0.4 to $5.3 \mathrm{R}_{\odot}$, with four-fifths of the sample falling between 0.5 and $2.5 \mathrm{R}_{\odot}$.

6. We have derived average radii for groups of stars with similar masses (i.e., in similar locations on the H-R diagram). The mean radii for stars within the same mass/temperature range do decrease as the stars get closer to the ZAMS, but in most cases the uncertainties in the mean radii are too large to show definitively that we have observed contraction within a given mass range. However, we find that the mean relative change in radius with luminosity for the five different mass/temperature ranges is $0.49 \pm 0.16$, meaning that we do find evidence for pre-main-sequence contraction in the overall sample of stars used at the three-sigma level.

In conclusion, combining $v \sin (i)$ measurements with photometric periods can yield valuable insight into the astrophysics of $\mathrm{T}$ Tauri stars. The methods used here allow us to test the luminosities and effective temperatures of $\mathrm{T}$ Tauri stars and thereby test their masses and ages, since these are usually derived by comparing $L$ and $T_{\text {eff }}$ with pre-main-sequence evolutionary models. Furthermore, even with a modest number of stars with good-quality $v \sin (i)$ values and periods, we have shown geometrically for the first time that the stars in our sample are undergoing contraction as they evolve toward the main sequence. Applying the method to even larger samples of stars with well-determined $v \sin (i)$ 's and periods is likely to improve our understanding of this important stage of low-mass stellar evolution.

K.L.R. gratefully acknowledges a grant from the Sigma Xi Scientific Research Society, which provided funding for the first observing run. We are grateful also to Lynne Hillenbrand for furnishing the auxiliary data so essential to the design and analysis of the project. We have benefited from useful discussions with Lynne Hillenbrand, Debra Fischer, and César Briceño. Chris Dolan provided advice and assistance with the spectral data reduction during the initial phase of the project. We would like to thank WIYN queue observers Di Harmer and Daryl Willmarth for executing the December 1997 observations. The H-R diagram with pre-main-sequence tracks shown in Figure 2 was made using plotting macros written by Karen Strom. Finally, we thank John Salzer, César Briceño, Lynne Hillenbrand, and the referee Steve Strom for providing useful comments and suggestions that improved the paper. This work was supported in part by a NASA grant through its Origins program to W.H. and by NSF grant AST-9417195 to R.D.M. 


\section{REFERENCES}

Andersen, J. 1991, A\&A Rev., 3, 91

Attridge, J. \& Herbst, W. 1992, ApJ, 398, L61

Bertout, C. 1989, ARA\&A, 27, 351

Bodenheimer, P. 1995, ARA\&A, 33, 199

Carpenter, J.M., Hillenbrand, L.A., \& Skrutskie, M.F. 2001, AJ, in press

Casey, B.W., Mathieu, R.D., Suntzeff, N.B., \& Walter, F.M. 1995, AJ, 109, 2156

Casey, B.W., Mathieu, R.D., Vaz, L.P.R., Andersen, J., \& Suntzeff, N.B. 1998, AJ, 115, 1617

Chandrasekhar, S., \& Münch, G. 1950, ApJ, 111, 142

Choi, P.I. \& Herbst W. 1996, AJ, 111, 283

Cohen, M. \& Kuhi, L.V. 1979, ApJS, 41, 743

Covino, E., Catalano, S., Frasca, A., Marilli, E., Fernandez, M., Alcala, J.M., Melo, C., Paladino, R., Sterzik, M.F., \& Stelzer, B. 2000, A\&A, 361, L49

D'Antona, F. \& Mazzitelli, I. 1994, ApJS, 90, 467

Duncan, D.K. 1993, ApJ, 406, 172

Gliese, W., \& Jahreiss, H. 1991, Third Catalogue of Nearby Stars (Astron. Rechen-Institut, Heidelberg)

Gray, D.F. 1992, The Observation and Analysis of Stellar Photospheres (Cambridge: Cambridge University Press)

Hartmann, L. 2001, AJ, 121, 1030

Hartmann, L., Hewett, R., Stahler, S., \& Mathieu, R.D. 1986, ApJ, 309, 275

Heiles, C., Goodman, A.A., McKee, C.F., Zweibel, E.G. 1993, in Protostars and Planets III, ed. E.H. Levy and J.I. Lunine (Tucson: University of Arizona Press), 279

Herbst, W. 1989, AJ, 98, 2268

Herbst, W., Rhode, K.L., Hillenbrand, L.A., \& Curran, G. 2000, AJ, 119, 261

Herbst, W., Bailer-Jones, C.A., Mundt, R., Meisenheimer, K. and Wackermann, R. 2001, A\&A, in preparation

Herbst, W., Herbst, D.K., Grossman, E.J., \& Weinstein, D. 1994, AJ, 108, 1906 
Hillenbrand, L.A. 1997, AJ, 113, 1733

Hillenbrand, L.A., Strom, S.E., Calvet, N., Merrill, K.M., Gatley, I., Makidon, R.B., Meyer, M.R., \& Skrutskie, M.F. 1998, AJ, 116, 1816

Jones, B.F. \& Walker, M.F. 1988, AJ, 95, 1755

King, J.R. 1998, AJ, 116, 254

Königl, A. 1991, ApJ, 370, L39

Mamajek, E.E., Lawson, W.A., \& Feigelson, E.D. 2000, ApJ, 544, 356

Mandel, G.N. \& Herbst, W. 1991, ApJ, 383, L75

Ostriker, E. \& Shu, F.W. 1995, ApJ, 447, 813

Padgett, D.L. 1996, ApJ, 471, 847

Popper, D.M. 1987, ApJ, 313, L81

Rebull, L.M. 2000, AJ, 121, 1676

Rydgren, A.E. \& Vrba, F.J. 1983, ApJ, 267, 191

Shu, F., Najita, J., Ostriker, E., Wilkin, F., Ruden, S., \& Lizano, S. 1994, ApJ, 429, 781

Soderblom, D.R., King, J.R., Siess, L., Jones, B.F., \& Fischer, D. 1999, AJ, 118, 1301

Stassun, K.G., Mathieu, R.D., Mazeh, T., \& Vrba, F.J. 1999, AJ, 117, 2941

Stauffer, J.R. 1991, in Angular Momentum Evolution of Young Stars, ed. S. Catalano and J.R. Stauffer (Dordrecht: Kluwer Academic Publishers), 117

Tonry, J. \& Davis, M. 1979, AJ, 84, 1511

Vogel, S.N. \& Kuhi, L.V. 1981, ApJ, 245, 960

Vrba, F.J., Rydgren, A.E., Chugainov, P.F., Shakovskaia, N.I., \& Zak, D.S. 1986, ApJ, 306, 199

Walker, M.F. 1969, ApJ, 155, 447

Weaver, W.B. 1987, ApJ, 319, L89

White, R.J., Ghez, A.M., Reid, I.N., Schultz, G. 1999, ApJ, 520, 811

Wolff, S.C., Strom, S.E., \& Hillenbrand, L.H. 2001, in preparation 


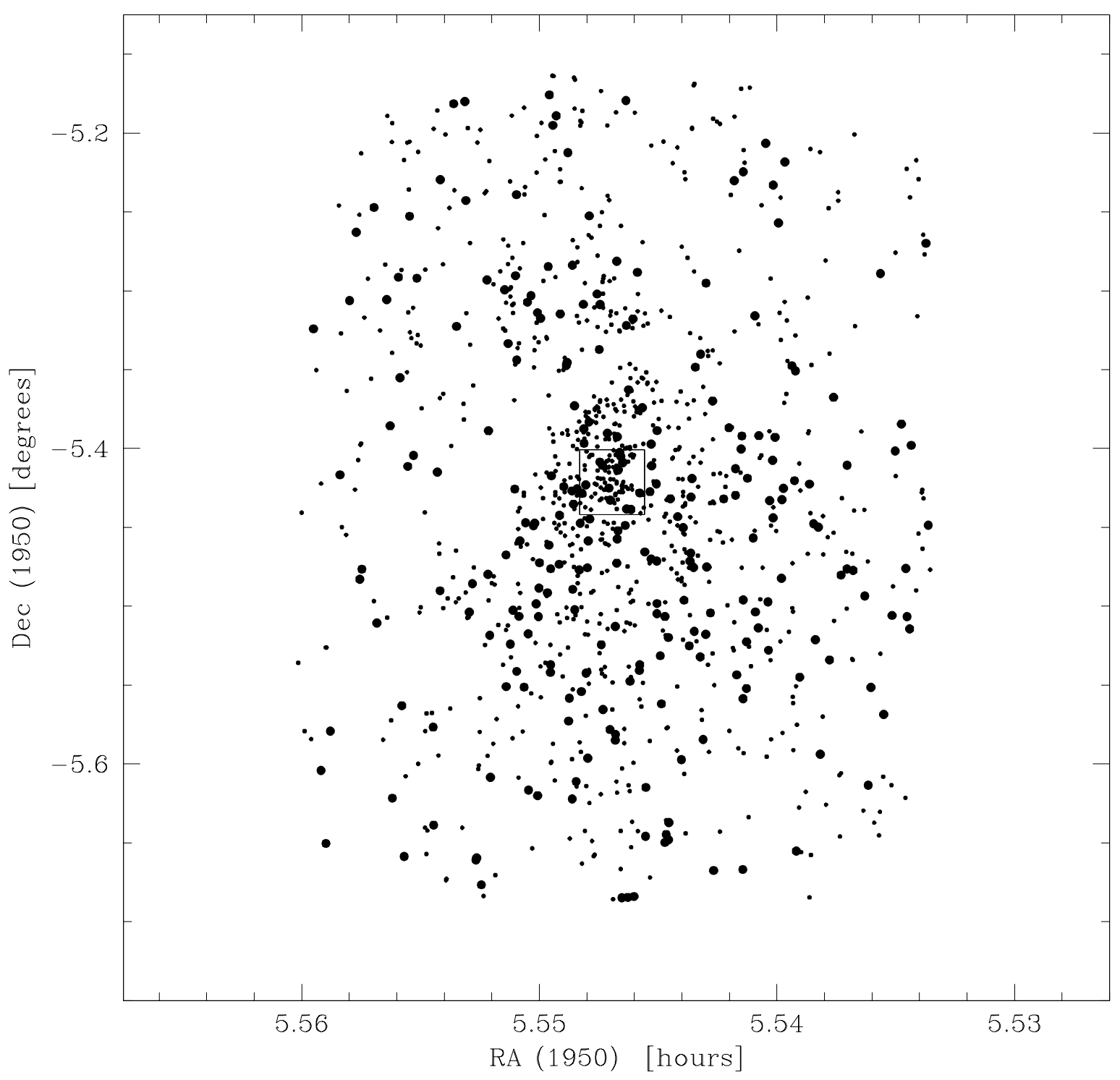

Fig. 1. - The locations on the sky of the 256 ONC stars observed spectroscopically for this study are shown here as filled circles. 1053 stars included in the Jones \& Walker (1988) proper motion survey are shown as small dots. The four Trapezium stars are located at the center of the small box in the middle of the field. 


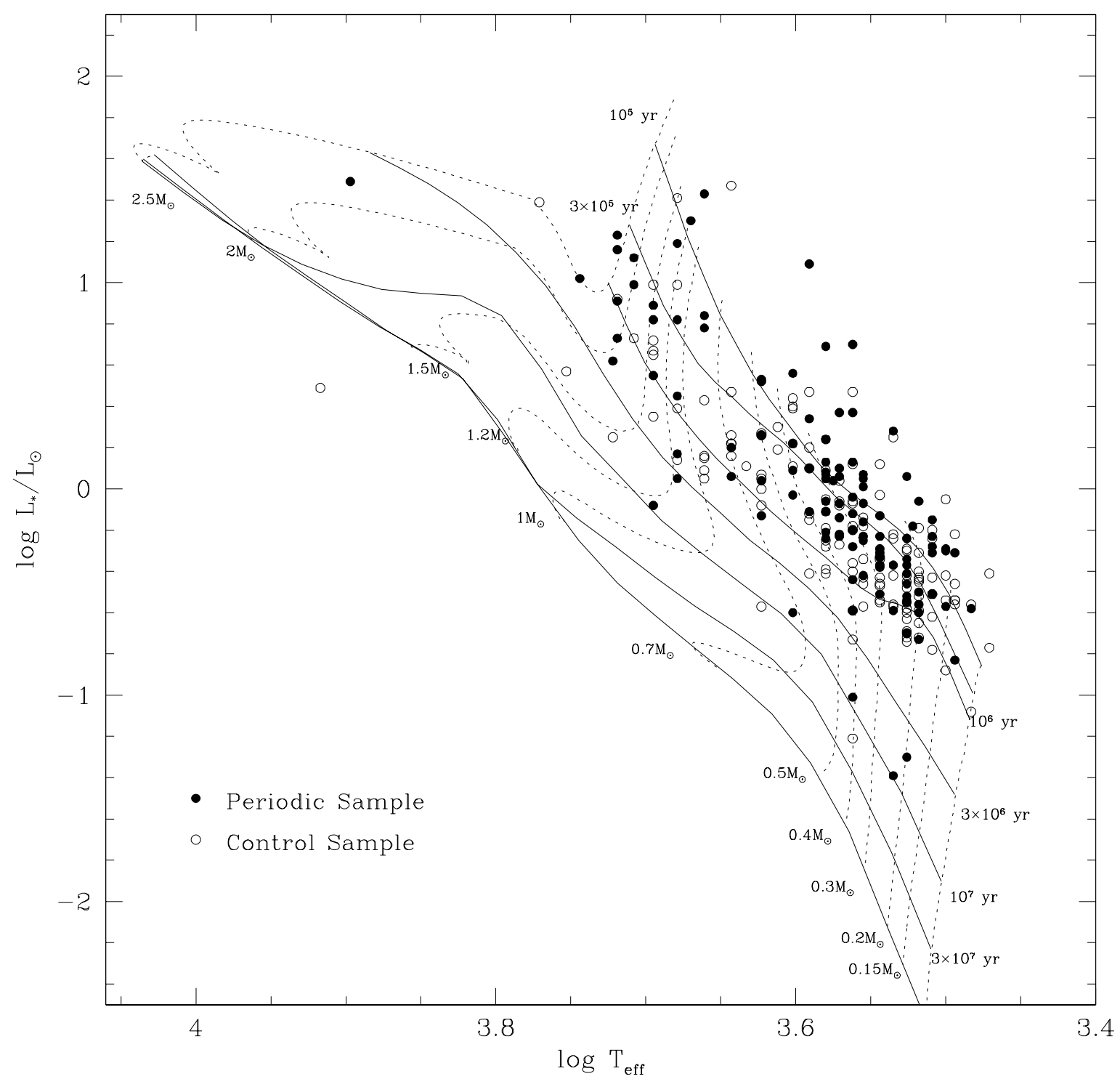

Fig. 2.- The positions on the H-R diagram of the 256 stars included in this study are shown here. Filled circles are stars in the Periodic sample, and open circles are Control sample stars. Luminosity and effective temperature values are from Hillenbrand (1997). Pre-main-sequence evolutionary tracks are from D'Antona \& Mazzitelli (1994, model 1). 


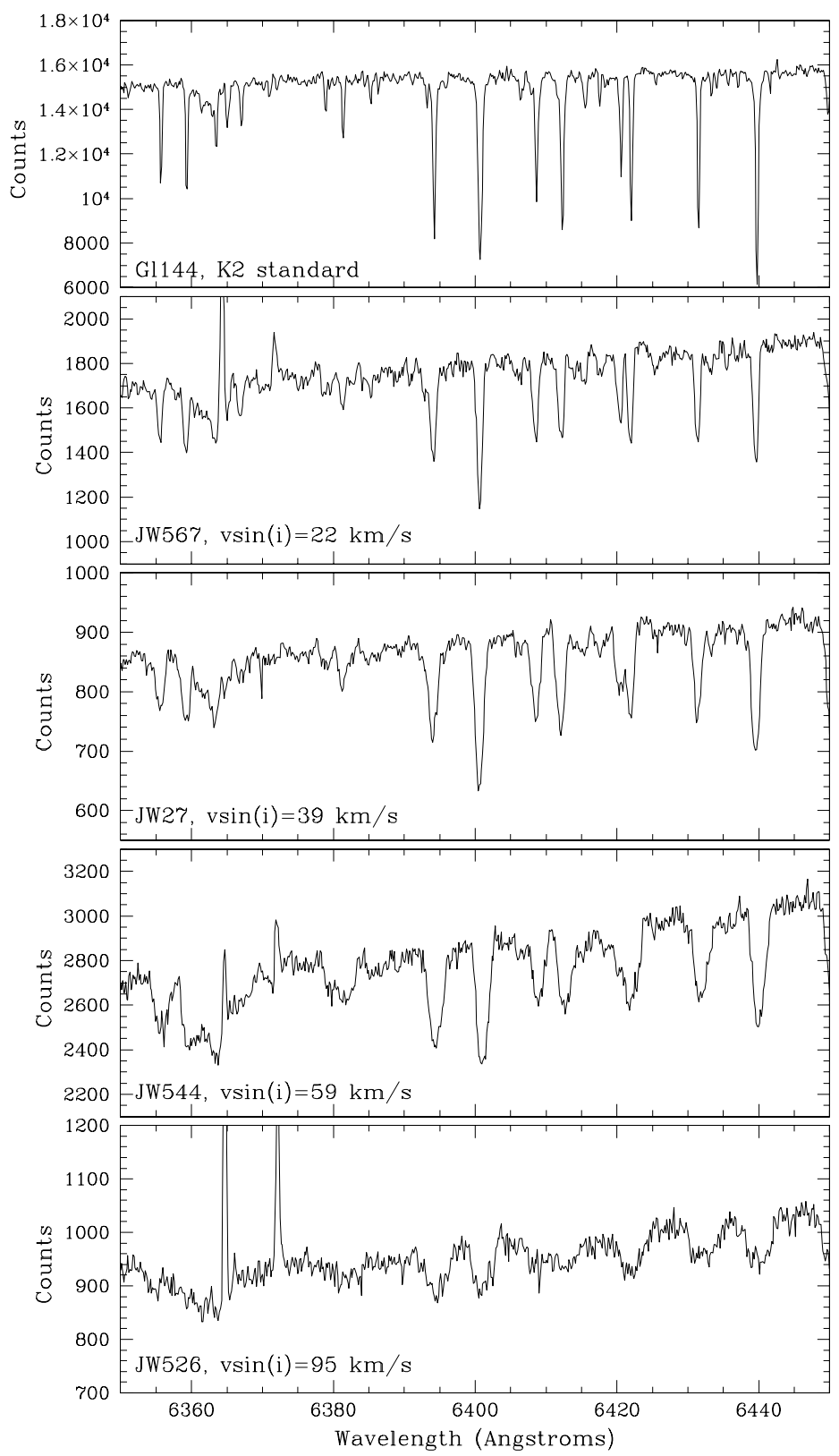

Fig. 3.- Examples of template star and target star spectra obtained for this study. The top spectrum is from Gliese 144, a K2V star used as a spectral template in the cross-correlation procedure. Its rotational velocity is below our $v \sin (i)$ measurement limit. The bottom four panels show spectra of Jones \& Walker (1988) stars with varying projected rotational velocities; the stellar absorption lines become broader and more shallow with increasing $v \sin (i)$. Emission lines from the Orion Nebula appear in some of the spectra. 


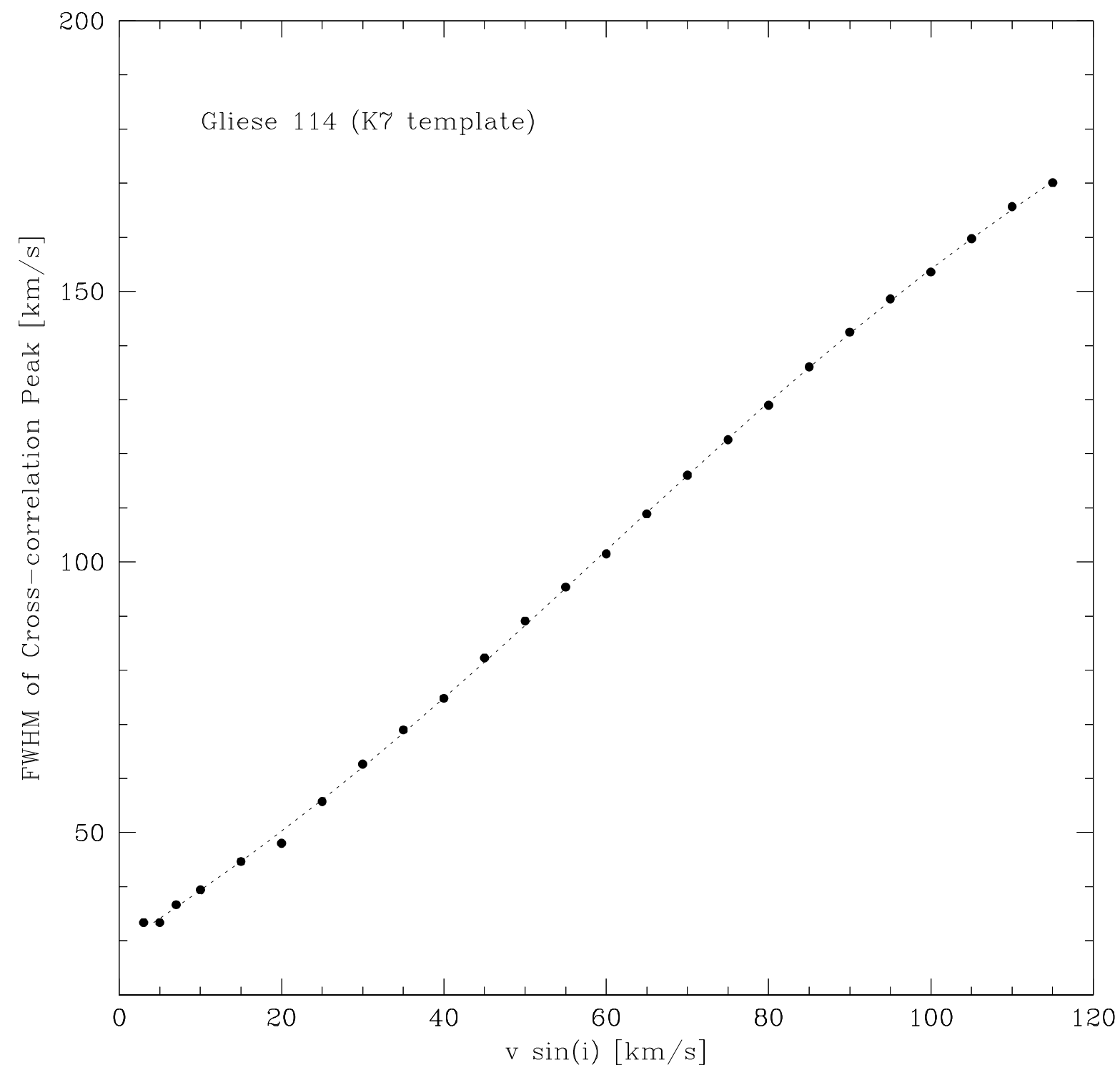

Fig. 4.- Calibration curve for the narrow-lined template star Gliese 114, created by crosscorrelating the original spectrum of this star against a series of artificially-broadened versions of the spectrum. The FWHM of the cross-correlation peak was measured and is plotted here against the velocity corresponding to each broadened spectrum (points). The polynomial fit to the FWHM vs. velocity data is shown as a dotted line. The relationship between the width of the peak and $v \sin (i)$ is fairly linear above $7 \mathrm{~km} \mathrm{~s}^{-1}$. 

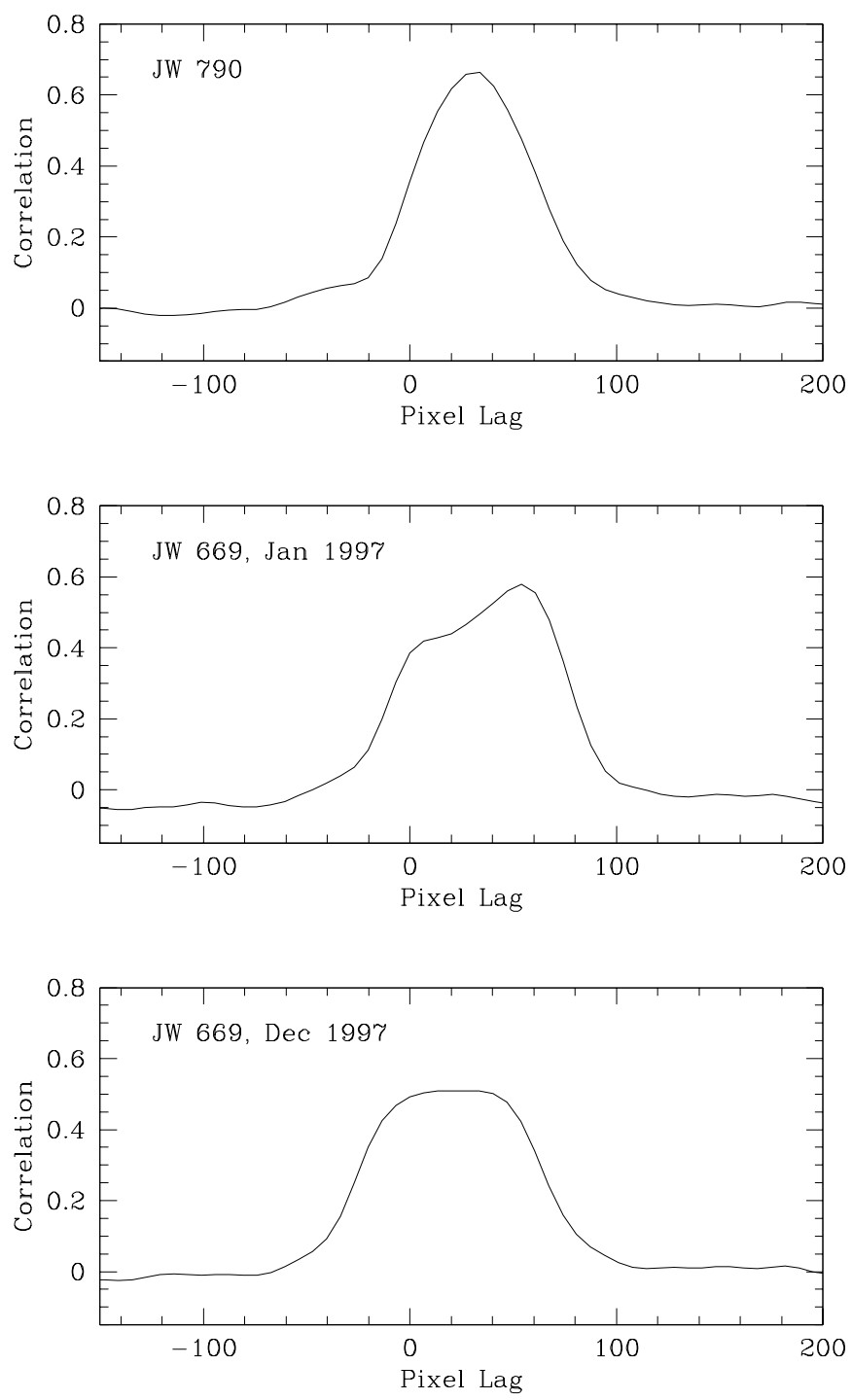

Fig. 5.- Shown here are the peaks of the cross-correlation functions for the target stars JW 790 (top panel) and JW 669 (bottom two panels). The cross-correlation functions are produced by cross-correlating the stars against the narrow-lined template star closest in effective temperature to the target. For JW 790, the cross-correlation peak is regular in shape and well-approximated by a Gaussian function. The peaks for JW 669, made using spectra taken in January 1997 and December 1997, appear more like two overlapping Gaussians, an indication that this star may be a spectroscopic binary. 

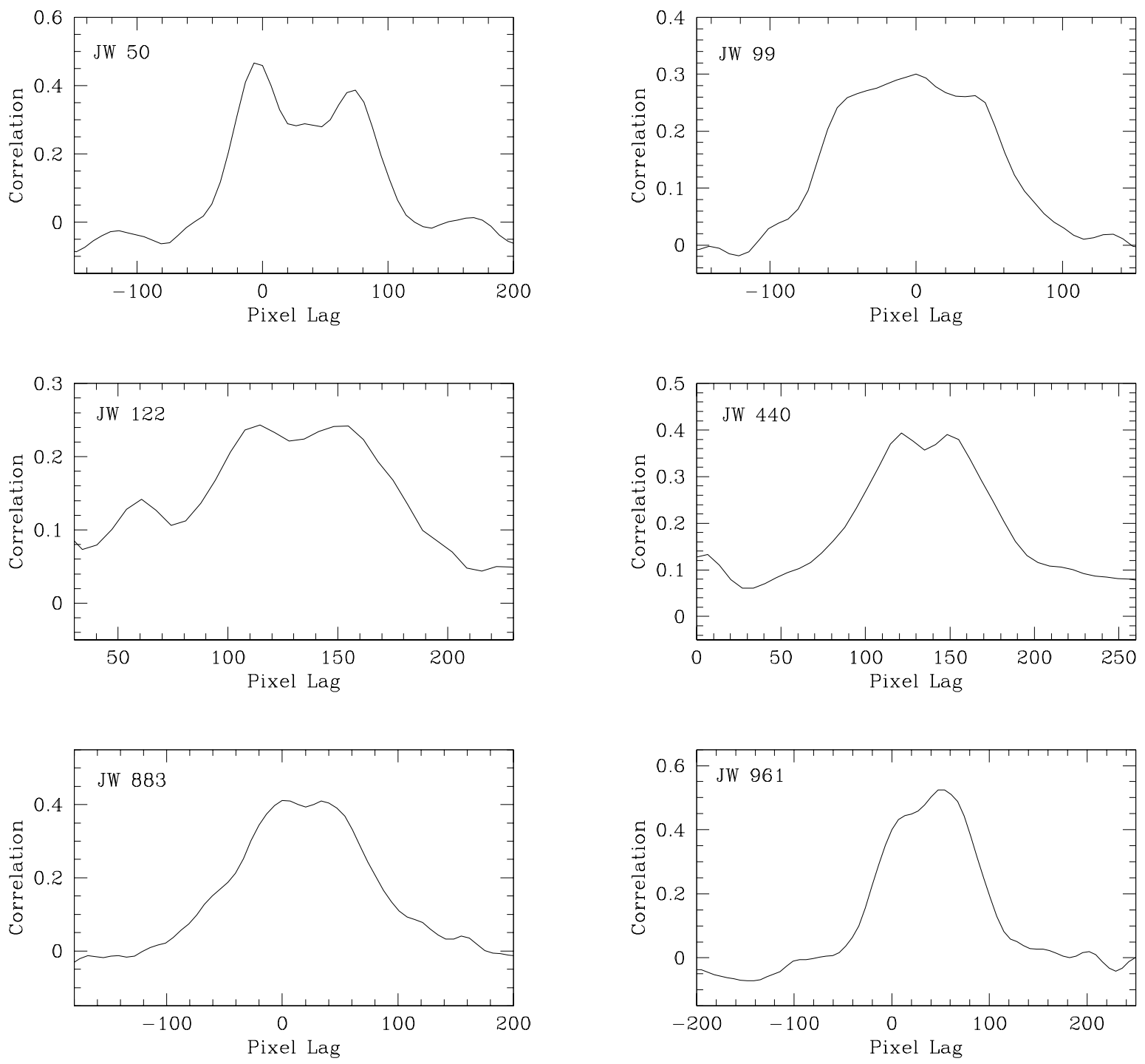

Fig. 6.- Plotted here are the cross-correlation function peaks for six of the seven double-lined spectroscopic binary candidates found in our data set. (The other SB2 candidate, JW 669, is shown in Figure 5.) These cross-correlation peaks show structure (sometimes appearing as multiple overlapping Gaussians) and are wider than typical peaks, possibly due to the presence of two sets of spectral lines in the target star spectra. 


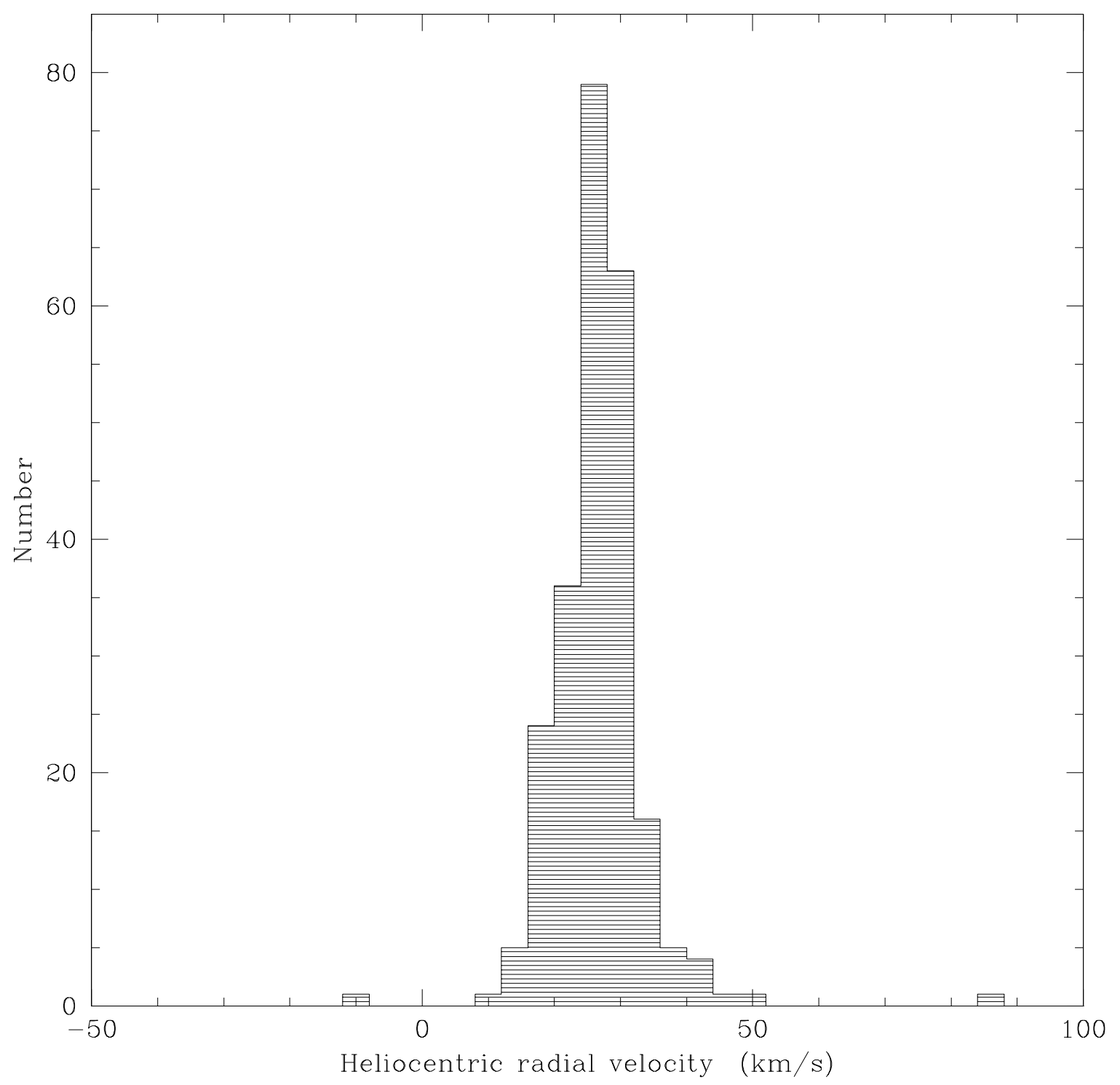

Fig. 7.- Histogram of the heliocentric radial velocity values for the stars for which $v \sin (i)$ was measured. The mean and standard deviation of the distribution are $26.7 \mathrm{~km} \mathrm{~s}^{-1}$ and $5.6 \mathrm{~km} \mathrm{~s}^{-1}$, respectively. 


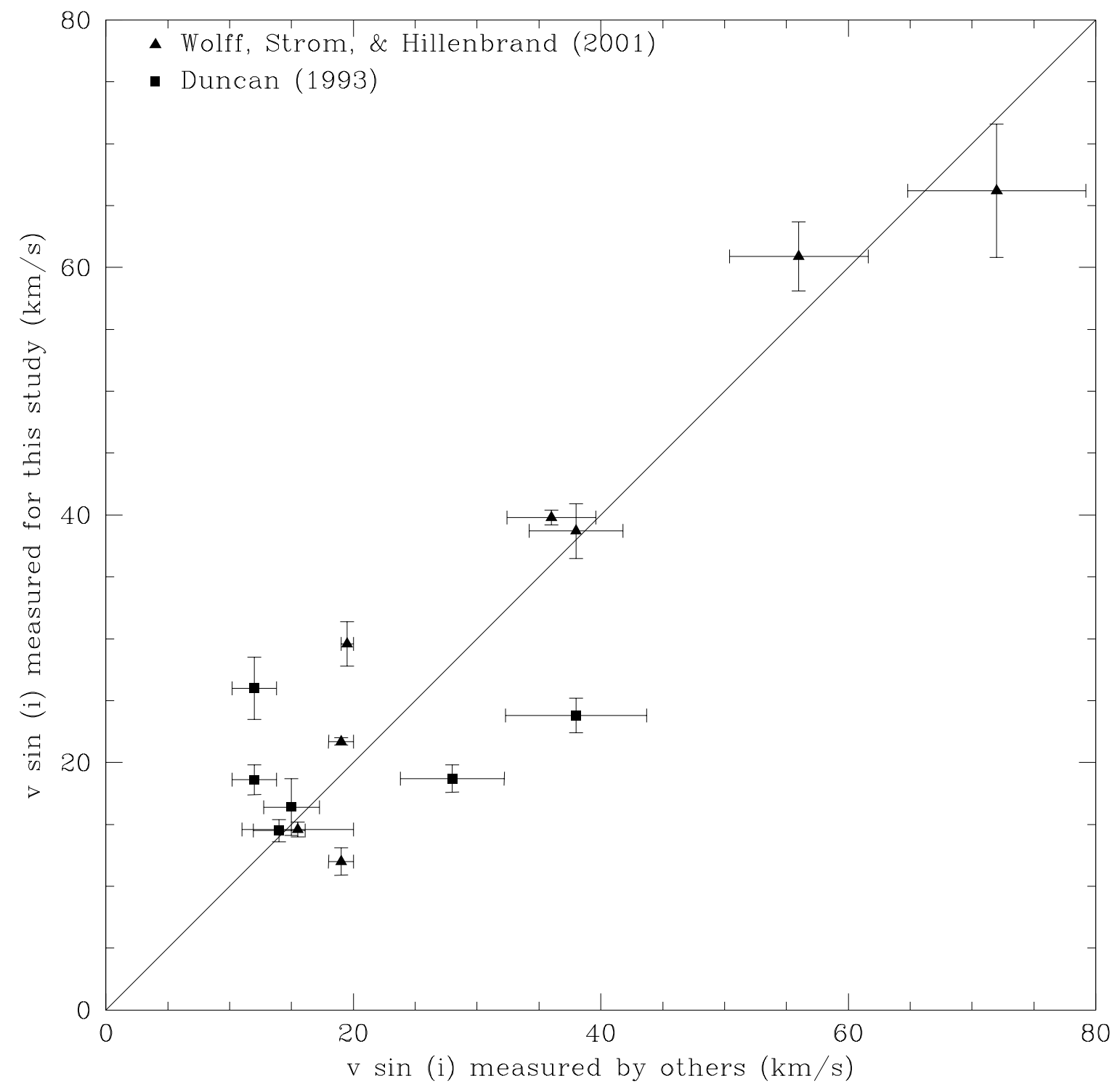

Fig. 8. - Comparison of $v \sin (i)$ values for 14 stars observed for this study and values from Duncan (1993) (squares) and Wolff, Strom, \& Hillenbrand (2001) (triangles). 
All Program Stars

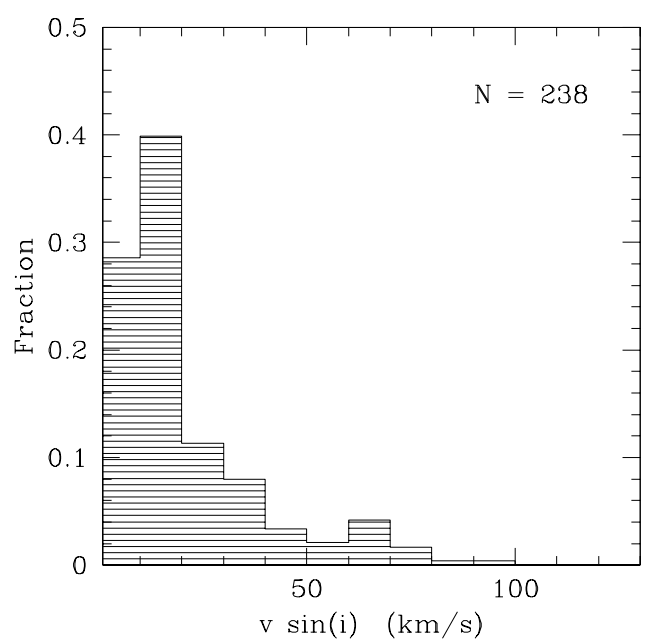

Periodic Sample

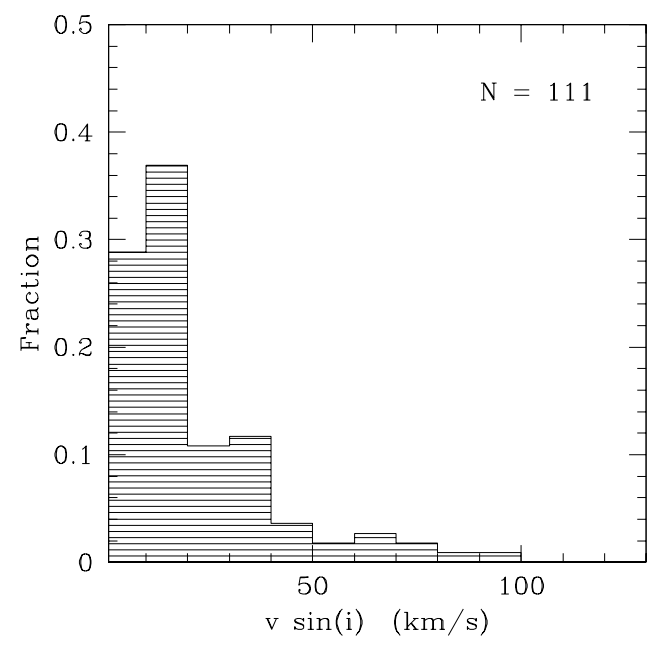

Control Sample

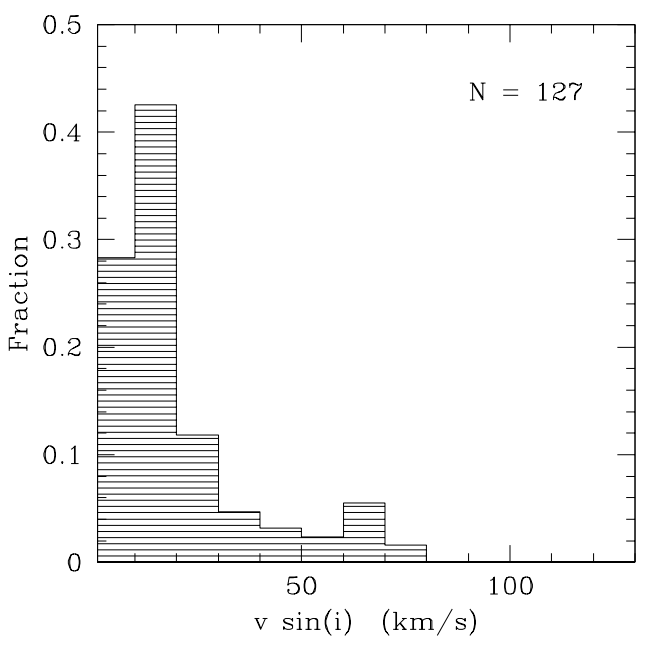

Fig. 9. - The top plot shows the distribution of $v \sin (i)$ values for the 238 stars for which $v \sin (i)$ was successfully measured. The bottom two plots are the $v \sin (i)$ distributions for the Periodic and Control sample stars. Although there appears to be a slight excess of rapid rotators in the Periodic sample, a K-S test comparing the $v \sin (i)$ distributions of the Periodic and Control samples shows that they are not significantly different from each other. 

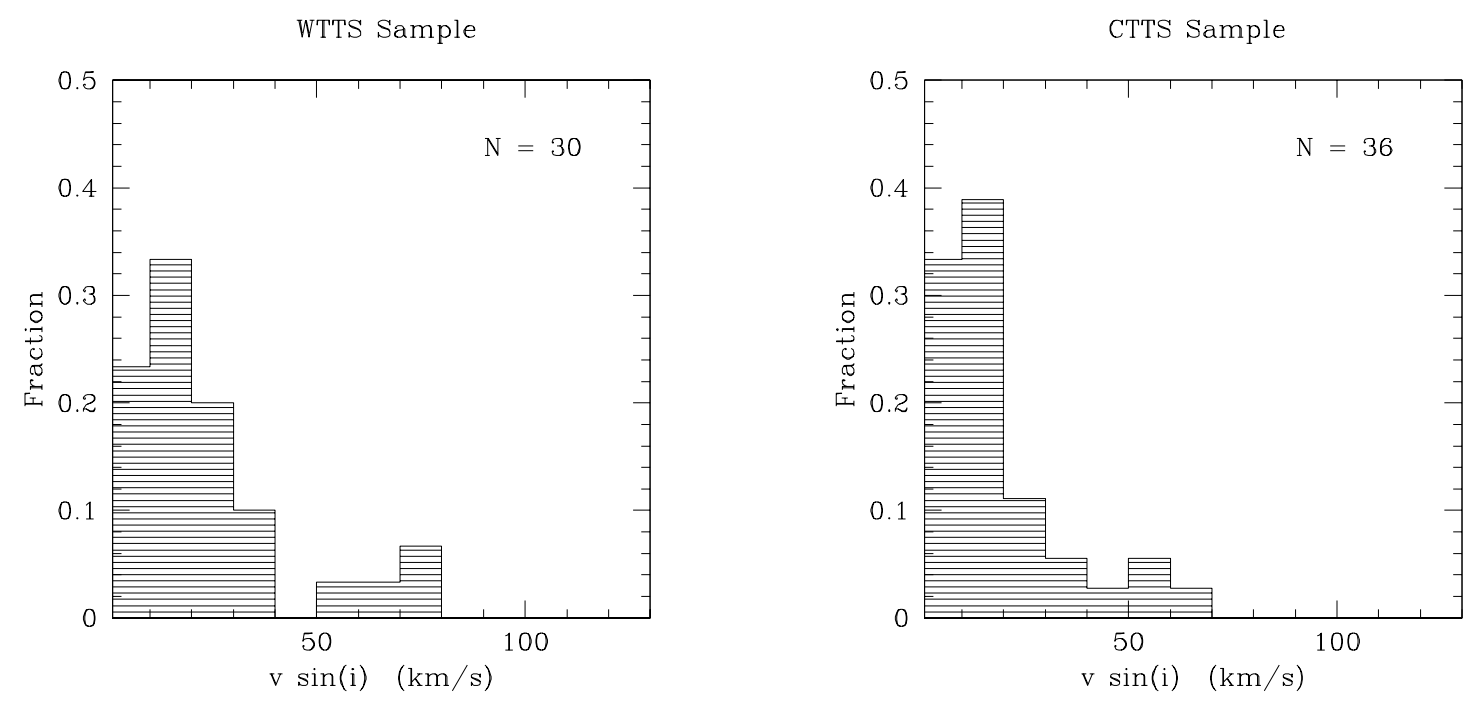

Fig. 10. - Comparison of the $v \sin (i)$ distributions for a sample of 36 stars likely to have accretion disks (CTTS) and 30 stars not likely to have disks (WTTS). The fraction of slow rotators is slightly higher for the CTTS sample, but a K-S test shows that the distributions do not differ significantly. 


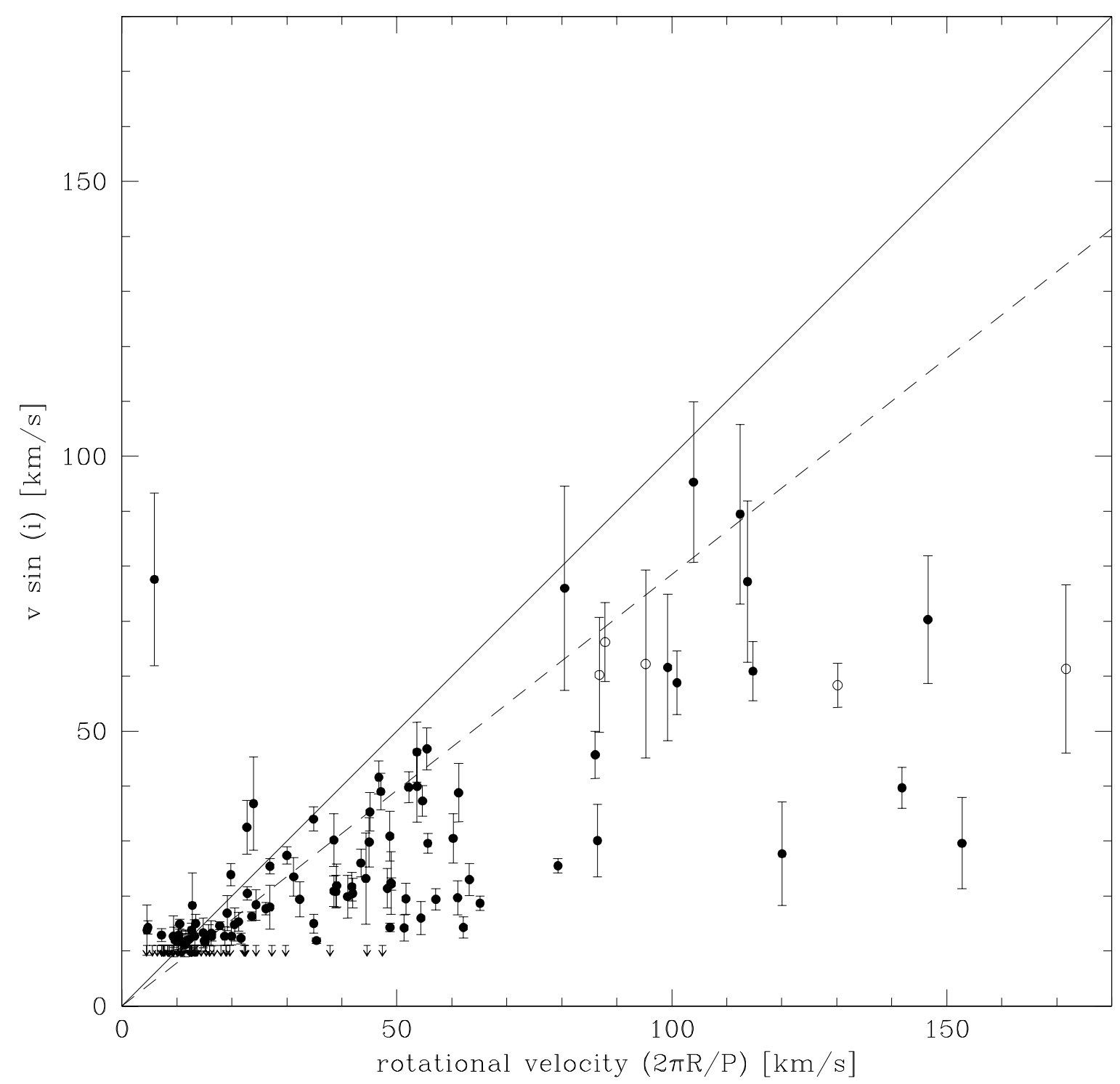

Fig. 11.- Measured $v \sin (i)$ versus equatorial rotation velocity for the 153 stars for which the latter quantity can be calculated. SB2 candidates are plotted with open circles, and $v \sin (i)$ values less than or equal to $11.0 \mathrm{~km} \mathrm{~s}^{-1}$ are shown as upper limits. Equatorial velocities are derived from the equation $v_{e q}=2 \pi R / P$. The solid line marks $v \sin (i)=v_{e q}$, and the dotted line marks $v \sin (i)$ $=(\pi / 4) v_{e q}$, where $(\pi / 4)$ is the expected average value of $\sin (i)$ for a random distribution of stellar rotation axes. 


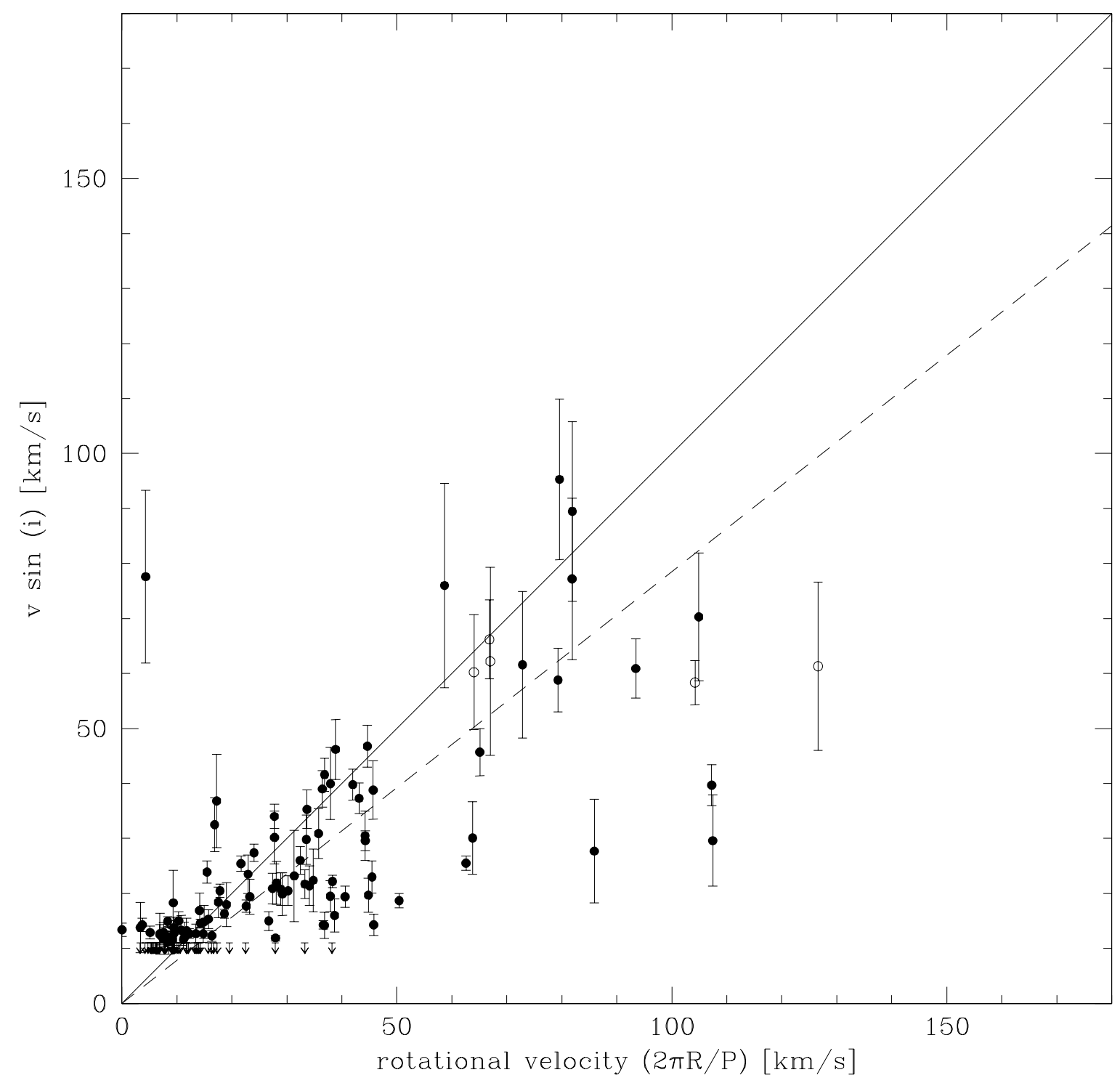

Fig. 12.- A new version of Figure 11: measured $v \sin (i)$ versus equatorial rotation velocity is shown for 153 stars. Here, the equatorial velocities have been calculated using $T_{\text {eff }}$ values that are $600 \mathrm{~K}$ hotter than published values. The points scatter around the dotted $v \sin (i)=(\pi / 4) v_{\text {eq }}$ line, as expected for a group of stars with randomly-oriented rotation axes. 


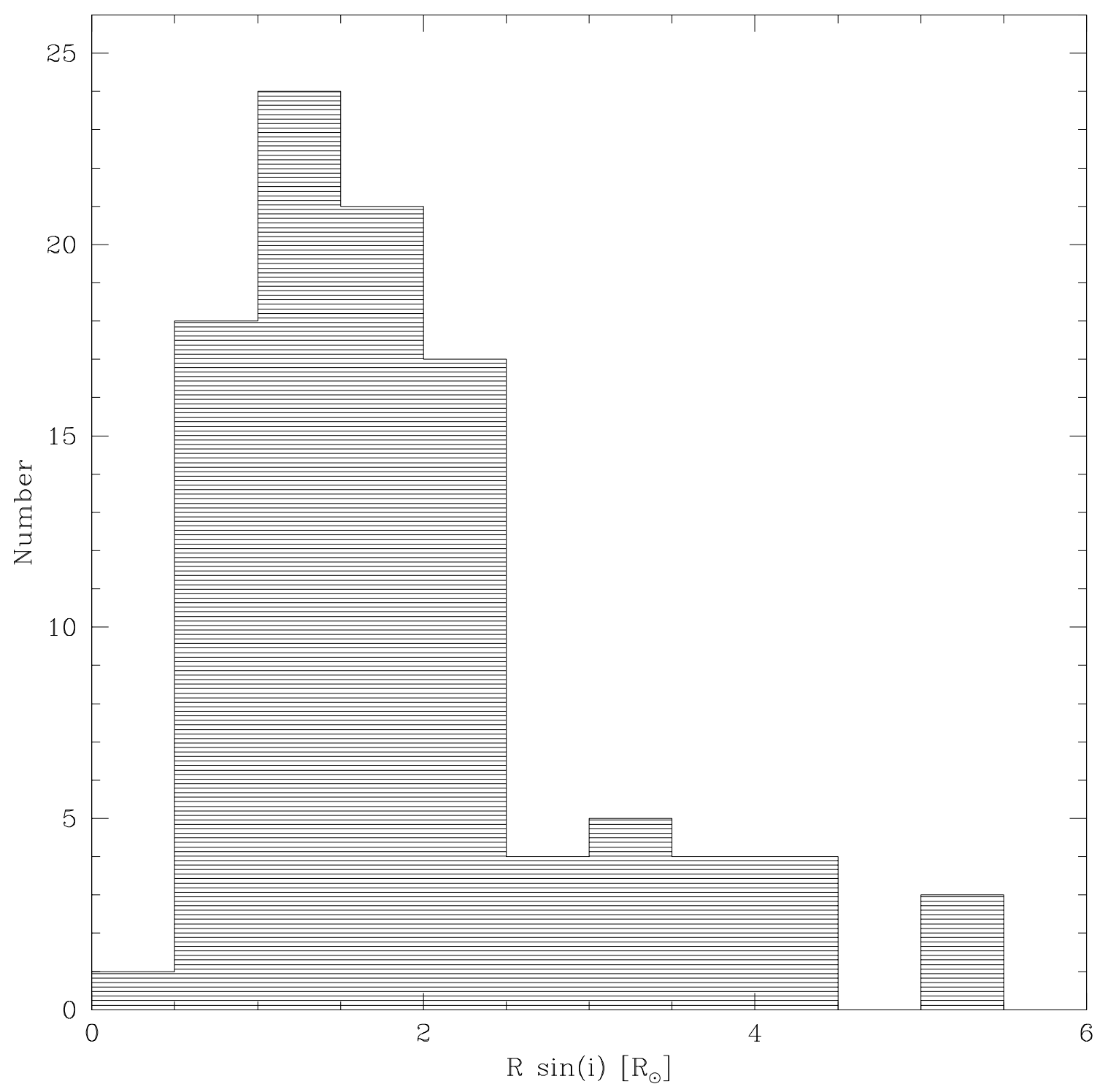

Fig. 13. - Minimum radii for 101 stars with measured period and $v \sin (i)>11.0 \mathrm{~km} \mathrm{~s}^{-1}$, calculated from the equation $R \sin (i)=P v \sin (i) /(2 \pi)$. $80 \%$ of the stars have $R \sin (i)<2.5 \mathrm{R}_{\odot}$. 


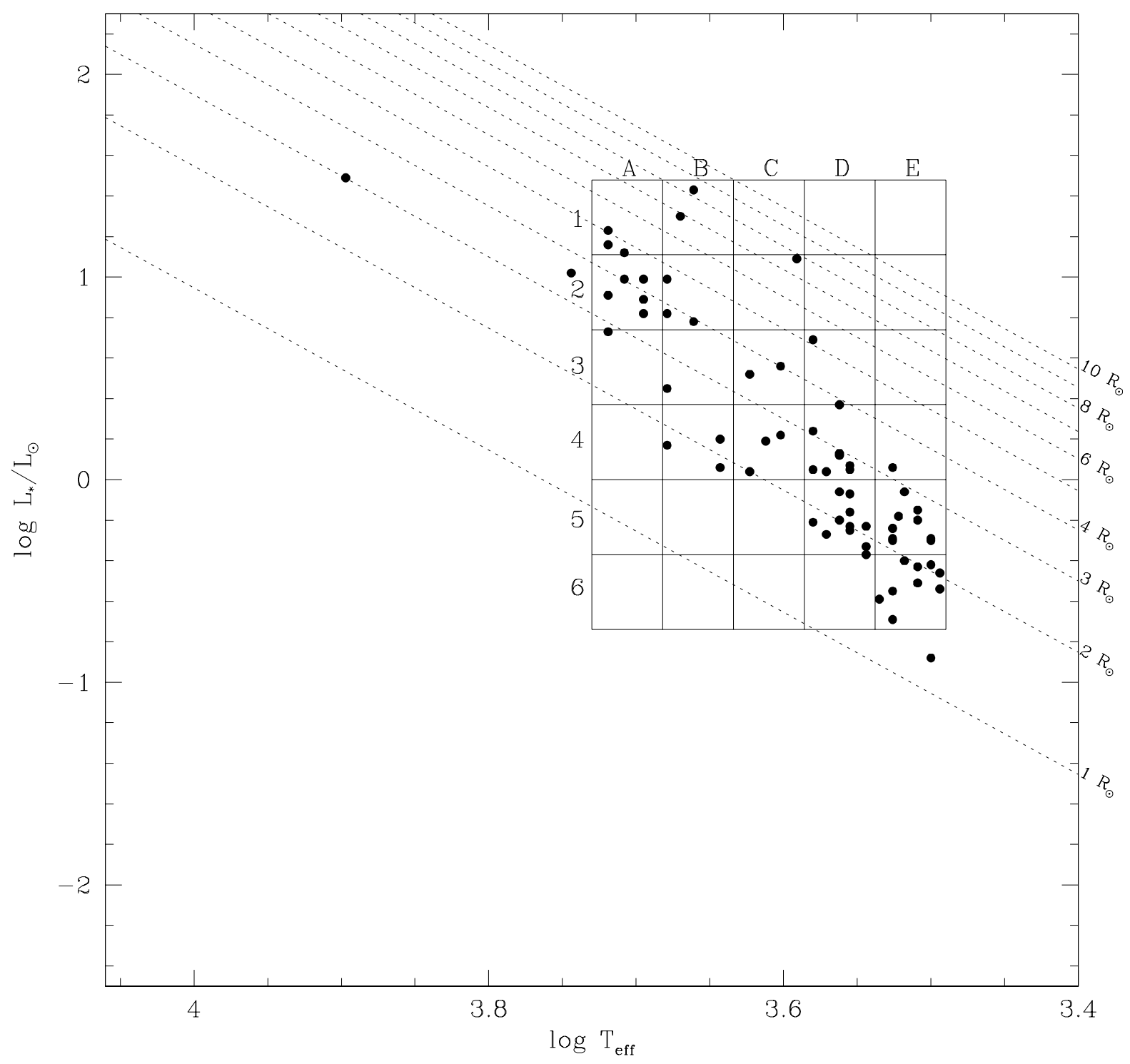

Fig. 14. - This figure shows the locations of the radii bins described in Section 3.2. The bins are marked with solid lines; each is 0.048 units wide in $\log T_{\text {eff }}$ and 0.37 units wide in $\log \left(L / L_{\odot}\right)$. The bin numbers are designated in the figure; e.g., the bin in the bottom right corner is bin E6. 68 stars with $v \sin (i) \geq 13.6 \mathrm{~km} \mathrm{~s}^{-1}$ and known rotation period, luminosity and effective temperature appear as filled circles. Dotted lines mark lines of constant radii in the log- $L, \log -T_{\text {eff }}$ plane, ranging from $1-10 \mathrm{R}_{\odot}$ in increments of 1.0. For bins containing two or more stars, average radii have been calculated and are given in Table 3. 
Table 1. ONC Stars Observed for this Study

\begin{tabular}{|c|c|c|c|c|c|c|c|c|c|c|c|c|c|c|}
\hline JW & Sam & $\begin{array}{c}\text { Mem } \\
(\%)\end{array}$ & $\begin{array}{c}I \\
(\mathrm{mag})\end{array}$ & $\begin{array}{c}V-I \\
(\mathrm{mag})\end{array}$ & $\begin{array}{c}\log (\mathrm{L}) \\
(\mathrm{L} \odot)\end{array}$ & $\begin{array}{c}\log \left(T_{\text {eff }}\right) \\
(\mathrm{K})\end{array}$ & $\begin{array}{c}\Delta(I-K) \\
\quad(\mathrm{mag})\end{array}$ & $\begin{array}{c}W_{\lambda}(\mathrm{CaII}) \\
(\AA)\end{array}$ & $\begin{array}{l}\text { Per } \\
\text { (days) }\end{array}$ & Src & $\begin{array}{c}v \sin (i) \\
\left(\mathrm{km} \mathrm{s}^{-1}\right)\end{array}$ & $\begin{array}{c}R \sin (i) \\
\quad\left(\mathrm{R}_{\odot}\right)\end{array}$ & SB2? & Date Obs \\
\hline 2 & $\mathrm{C}$ & 97 & 12.2 & 1.61 & 0.26 & 3.643 & 0.42 & $\ldots$. & & $\ldots$ & $\leq 11.0$ & & .. & Jan 97, Dec 97 \\
\hline 3 & $\mathrm{C}$ & 0 & 13.0 & 2.50 & 0.24 & 3.580 & 0.55 & 2.0 & 3.43 & $\mathrm{E}$ & $29.8 \pm 4.5$ & 2.02 & .. & Dec 97 \\
\hline 15 & $\mathrm{C}$ & 99 & 14.1 & 2.66 & -0.18 & 3.562 & 0.11 & 1.7 & 9.56 & $\mathrm{E}$ & $\leq 11.0$ & $\ldots$. & .. & Dec 97 \\
\hline 17 & $\mathrm{C}$ & 99 & 13.5 & 3.00 & -0.20 & 3.509 & 0.10 & 0.8 & 3.14 & $\mathrm{E}$ & $19.9 \pm 3.9$ & 1.23 & .. & Dec 98 \\
\hline 18 & $\mathrm{C}$ & 99 & 14.4 & 2.66 & -0.46 & 3.544 & 0.54 & 0.6 & $\ldots$ & $\ldots$ & $13.1 \pm 3.3$ & $\ldots$ & .. & Dec 97 \\
\hline 20 & $\mathrm{C}$ & 99 & 13.8 & 2.67 & -0.40 & 3.518 & 0.10 & 0.7 & 0.67 & $\mathrm{E}$ & $70.3 \pm 11.6$ & 0.93 & .. & Dec 98 \\
\hline 22 & $\mathrm{C}$ & 99 & 13.9 & 2.38 & -0.34 & 3.555 & 0.14 & 2.2 & & $\ldots$ & $\leq 11.0$ & & .. & Dec 97 \\
\hline 25 & $\mathrm{C}$ & 99 & 15.2 & 2.54 & -0.88 & 3.500 & 0.52 & 0.0 & 2.28 & $\mathrm{E}$ & $18.0 \pm 4.0$ & 0.81 & .. & Dec 98 \\
\hline 27 & $\mathrm{C}$ & 93 & 11.1 & 1.31 & 0.72 & 3.695 & 0.22 & $\ldots$. & & $\ldots$ & $38.7 \pm 2.2$ & $\ldots$ & .. & Dec 97 \\
\hline 29 & $\mathrm{C}$ & 97 & 11.7 & 1.60 & 0.67 & 3.695 & 0.32 & $\ldots$ & $\ldots$ & $\ldots$ & $23.8 \pm 1.4$ & $\ldots$. & .. & Jan 97 \\
\hline 31 & $\mathrm{C}$ & 99 & 14.2 & 2.44 & -0.58 & 3.526 & 0.01 & 1.4 & $\ldots$ & $\ldots$ & $\leq 11.0$ & $\ldots$ & .. & Dec 98 \\
\hline 36 & $\mathrm{C}$ & 99 & 14.3 & 3.08 & -0.30 & 3.526 & 0.46 & -4.1 & 2.72 & $\mathrm{E}$ & $21.9 \pm 3.9$ & 1.18 & .. & Dec 98 \\
\hline 37 & $\mathrm{C}$ & 92 & 12.9 & 1.68 & 0.14 & 3.679 & 0.62 & -8.4 & 8.27 & $\mathrm{E}$ & $14.9 \pm 0.9$ & 2.43 & .. & Jan 97 \\
\hline 39 & $\mathrm{C}$ & 99 & 14.3 & 2.40 & -0.63 & 3.526 & 0.14 & 0.8 & $\ldots$ & $\ldots$ & $11.7 \pm 1.1$ & $\ldots$. & .. & Dec 98 \\
\hline 46 & $\mathrm{C}$ & 97 & 11.1 & 1.27 & 0.73 & 3.708 & 0.10 & $\ldots$ & $\ldots$ & $\ldots$ & $14.6 \pm 0.6$ & $\ldots$ & .. & Dec 97 \\
\hline 50 & $\mathrm{C}$ & 98 & 11.7 & 1.75 & 0.44 & 3.602 & 1.37 & -14.6 & $\ldots$ & $\ldots$ & $16.4 \pm 2.3$ & $\ldots$ & $\mathrm{Y}$ & Dec 98 \\
\hline 51 & $\mathrm{C}$ & 99 & 14.4 & 2.37 & -0.57 & 3.555 & 1.05 & 0.0 & 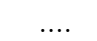 & $\ldots$ & $12.4 \pm 1.5$ & $\ldots$ & .. & Dec 98 \\
\hline 55 & $\mathrm{C}$ & 95 & 14.4 & 2.67 & -0.60 & 3.526 & 0.36 & 1.0 & $\ldots$ & $\ldots$ & $\leq 11.0$ & $\ldots$ & .. & Dec 97 \\
\hline 63 & $\mathrm{C}$ & 99 & 12.8 & 2.06 & 0.26 & 3.623 & 0.20 & 1.9 & $\ldots$ & $\ldots$ & $17 . \overline{4} \pm 1.2$ & $\ldots$ & .. & Jan 97 \\
\hline 65 & $\mathrm{P}$ & 99 & 13.8 & 2.25 & -0.24 & 3.580 & 0.50 & 0.0 & 7.39 & $\mathrm{~V}$ & $12.0 \pm 1.1$ & 1.75 & .. & Dec 97 \\
\hline 73 & $\mathrm{C}$ & 99 & 14.0 & 1.85 & -0.59 & 3.562 & 1.65 & -34.0 & $\ldots$ & $\ldots$ & $\ldots \ldots \ldots$ & $\ldots$ & .. & Dec 98 \\
\hline 75 & $\mathrm{P}$ & 99 & 11.1 & 1.61 & 0.89 & 3.695 & 0.09 & $\ldots$ & 3.45 & V & $29.6 \pm 1.8$ & 2.02 & .. & Jan 97 \\
\hline 76 & $\mathrm{P}$ & 99 & 13.6 & 2.97 & -0.23 & 3.509 & 0.16 & 1.3 & 6.33 & $\mathrm{~V}$ & $\leq 11.0$ & $\ldots$ & .. & Jan 97 \\
\hline 77 & $\mathrm{P}$ & 99 & 13.6 & 2.16 & -0.21 & 3.580 & 0.42 & 1.8 & 1.50 & EVS & $38 . \overline{8} \pm 5.3$ & 1.15 & .. & Dec 97 \\
\hline 83 & $\mathrm{P}$ & 99 & 14.7 & 3.12 & -0.29 & 3.544 & 0.37 & -9.2 & 7.72 & $\mathrm{~V}$ & $18.3 \pm 5.9$ & 2.79 & .. & Dec 97 \\
\hline 91 & $\mathrm{C}$ & 99 & 13.5 & 2.44 & -0.24 & 3.509 & -0.06 & 1.2 & 17.08 & $\mathrm{E}$ & $12.9 \pm 1.2$ & 4.35 & .. & Dec 97 \\
\hline 95 & $\mathrm{C}$ & 95 & 12.8 & 1.77 & -0.12 & 3.591 & 0.22 & 0.0 & $\cdots$ & $\ldots$ & $61.5 \pm 9.0$ & $\ldots$ & .. & Dec 97 \\
\hline 98 & $\mathrm{C}$ & 99 & 14.6 & 3.20 & -0.51 & 3.509 & 0.17 & 0.0 & 2.34 & $\mathrm{E}$ & $20.9 \pm 2.8$ & 0.97 & .. & Dec 98 \\
\hline 99 & $\mathrm{P}$ & 99 & 13.1 & 2.51 & 0.13 & 3.562 & 0.50 & 1.4 & 1.70 & $\mathrm{~V}$ & $60.2 \pm 10.5$ & 2.02 & $\mathrm{Y}$ & Jan 97,Dec 97 \\
\hline 106 & $\mathrm{C}$ & 99 & 13.4 & 2.42 & -0.29 & 3.526 & 0.10 & 1.5 & 1.70 & $\mathrm{E}$ & $23.0 \pm 2.9$ & 0.77 &.. & Dec 98 \\
\hline 111 & $\mathrm{C}$ & 98 & 13.0 & 1.83 & -0.15 & 3.591 & 0.24 & 2.2 & 4.94 & $\mathrm{E}$ & $\leq 11.0$ & $\ldots$ & .. & Jan 97 \\
\hline 114 & $\mathrm{P}$ & 99 & 15.3 & 2.14 & -1.01 & 3.562 & 2.01 & -1.4 & 8.73 & $\mathrm{~V}$ & $13 . \overline{8} \pm 4.6$ & 2.38 & .. & Dec 97 \\
\hline 116 & $\mathrm{P}$ & 99 & 12.1 & 1.44 & 0.20 & 3.643 & 0.09 & 1.6 & 2.34 & V & $39.0 \pm 3.3$ & 1.80 & .. & Jan 97 \\
\hline 117 & $\mathrm{C}$ & 99 & 13.2 & 2.13 & -0.05 & 3.580 & 0.77 & -6.3 & 8.87 & $\mathrm{E}$ & $\leq 11.0$ & $\ldots$ & .. & Jan 97 \\
\hline 122 & $\mathrm{C}$ & 98 & 14.4 & 3.02 & -0.54 & 3.494 & -0.17 & 0.0 & 0.98 & $\mathrm{E}$ & $62 . \overline{2} \pm 17.1$ & 1.20 & $\mathrm{Y}$ & Dec 97 \\
\hline 124 & $\mathrm{C}$ & 99 & 13.9 & 2.76 & -0.43 & 3.518 & -0.02 & 0.8 & & $\ldots$ & $14.7 \pm 3.8$ & $\ldots$ & .. & Dec 97 \\
\hline 126 & $\mathrm{C}$ & 99 & 14.4 & 2.55 & -0.69 & 3.526 & 0.01 & 0.8 & 2.78 & $\mathrm{E}$ & $18.4 \pm 2.8$ & 1.01 & .. & Dec 97 \\
\hline 127 & $\mathrm{C}$ & 99 & 14.3 & 2.94 & -0.45 & 3.518 & 1.05 & -2.8 & $\ldots$ & $\ldots$ & $13.9 \pm 2.1$ & $\ldots$ & .. & Dec 97 \\
\hline 128 & $\mathrm{P}$ & 97 & 12.6 & 2.82 & 0.28 & 3.535 & 0.32 & 0.0 & 8.83 & $\mathrm{~V}$ & $\leq 11.0$ & $\ldots$ & .. & Dec 97 \\
\hline 133 & $\mathrm{P}$ & 99 & 13.2 & 2.08 & -0.25 & 3.555 & 0.26 & 1.6 & 2.02 & V & $30 . \overline{9} \pm 4.5$ & 1.23 & .. & Dec 97 \\
\hline 135 & $\mathrm{P}$ & 99 & 14.4 & 2.93 & -0.46 & 3.526 & 1.11 & -2.3 & 3.69 & $\mathrm{~V}$ & $36.8 \pm 8.5$ & 2.68 & .. & Dec 97 \\
\hline 136 & $\mathrm{P}$ & 99 & 13.8 & 2.81 & 0.06 & 3.571 & $\ldots$ & $\ldots$ & 8.65 & $\mathrm{~V}$ & $11.6 \pm 1.7$ & 1.98 & .. & Dec 98 \\
\hline 140 & $\mathrm{C}$ & 99 & 13.9 & 3.10 & -0.19 & 3.518 & -0.29 & 3.8 & 4.58 & $\mathrm{E}$ & $\leq 11.0$ & $\ldots$ & .. & Dec 98 \\
\hline 146 & $\mathrm{C}$ & 99 & 13.5 & 2.27 & -0.33 & 3.544 & 0.32 & 0.0 & & $\ldots$ & $11.2 \pm 1.3$ & $\ldots$ & .. & Dec 98 \\
\hline 148 & $\mathrm{P}$ & 99 & 15.4 & 3.73 & 0.07 & 3.580 & 0.08 & 1.6 & 1.37 & VS & .......... & $\ldots$ & .. & Dec 97 \\
\hline 149 & $\mathrm{P}$ & 99 & 15.6 & 3.25 & -0.70 & 3.526 & 0.24 & -23.7 & 2.83 & $\mathrm{~V}$ & $\ldots \ldots .$. & $\ldots$ & .. & Dec 97 \\
\hline 150 & $\mathrm{C}$ & 99 & 14.0 & 2.87 & 0.27 & 3.623 & 1.13 & -1.7 & $\ldots$ & $\ldots$ & $\leq 11.0$ & $\ldots$ & .. & Dec 98 \\
\hline 152 & $\mathrm{C}$ & 99 & 14.1 & 2.78 & -0.42 & 3.526 & 0.39 & 2.6 & $\ldots$ & $\ldots$ & $\ldots \ldots .$. & $\ldots$ & .. & Dec 98 \\
\hline
\end{tabular}


Table 1-Continued

\begin{tabular}{|c|c|c|c|c|c|c|c|c|c|c|c|c|c|c|}
\hline JW & Sam & $\begin{array}{c}\text { Mem } \\
(\%)\end{array}$ & $\begin{array}{c}I \\
(\mathrm{mag})\end{array}$ & $\begin{array}{c}V-I \\
(\mathrm{mag})\end{array}$ & $\begin{array}{c}\log (\mathrm{L}) \\
(\mathrm{L} \odot)\end{array}$ & $\begin{array}{c}\log \left(\mathrm{T}_{\text {eff }}\right) \\
(\mathrm{K})\end{array}$ & $\begin{array}{c}\Delta(I-K) \\
\quad(\mathrm{mag})\end{array}$ & $\begin{array}{c}W_{\lambda}(\mathrm{CaII}) \\
(\AA)\end{array}$ & $\begin{array}{c}\text { Per } \\
\text { (days) }\end{array}$ & Src & $\begin{array}{c}v \sin (i) \\
\left(\mathrm{km} \mathrm{s}^{-1}\right)\end{array}$ & $\begin{array}{l}R \sin (i) \\
\quad\left(\mathrm{R}_{\odot}\right)\end{array}$ & SB2? & Date Obs \\
\hline 157 & $\mathrm{P}$ & 99 & 10.2 & 1.56 & 1.19 & 3.679 & 0.11 & $\ldots$. & 17.40 & $\mathrm{~V}$ & $\leq 11.0$ & $\ldots$ & .. & Dec 98 \\
\hline 158 & $\mathrm{P}$ & 99 & 13.3 & 2.15 & -0.23 & 3.555 & 0.71 & 1.5 & 1.95 & V & $19.5 \pm 2.8$ & 0.75 & .. & Jan 97 \\
\hline 159 & $\mathrm{P}$ & 99 & 13.9 & 2.46 & -0.37 & 3.544 & -1.72 & 0.3 & 1.12 & V & $76.0 \pm 18.6$ & 1.68 & .. & Dec 97 \\
\hline 163 & $\mathrm{C}$ & 99 & 11.8 & 1.30 & 0.22 & 3.643 & 0.53 & 1.6 & $\ldots$. & $\ldots$ & $\leq 11.0$ & $\ldots$ & .. & Dec 97 \\
\hline 164 & $\mathrm{C}$ & 99 & 14.0 & 2.38 & -0.47 & 3.544 & 0.63 & -0.7 & 6.52 & $\mathrm{E}$ & $12.2 \pm 1.6$ & 1.57 & .. & Dec 98 \\
\hline 165 & $\mathrm{P}$ & 97 & 11.7 & 1.82 & 1.49 & 3.897 & 0.11 & $\ldots$ & 5.77 & $\mathrm{~V}$ & $17.7 \pm 1.1$ & 2.02 & .. & Dec 98 \\
\hline 166 & C & 99 & 14.2 & 2.81 & -0.46 & 3.494 & -0.26 & 0.0 & 0.67 & $\mathrm{E}$ & $29.6 \pm 8.3$ & 0.39 & .. & Dec 97 \\
\hline 167 & $\mathrm{P}$ & 99 & 14.9 & 3.08 & -0.03 & 3.602 & 0.86 & 1.4 & 3.43 & $\mathrm{E}$ & $\leq 11.0$ & $\ldots$ & .. & Jan 97 \\
\hline 169 & $\mathrm{P}$ & 99 & 15.9 & $\ldots$ & -1.30 & 3.526 & $\ldots$ & $\ldots$. & 3.85 & $\mathrm{~V}$ & $\ldots \ldots \ldots$ & $\ldots$ & .. & Dec 98 \\
\hline 174 & $\mathrm{P}$ & 99 & 14.1 & 2.40 & -0.59 & 3.535 & 0.12 & 3.7 & 1.36 & EVS & $46.2 \pm 5.4$ & 1.24 & .. & Jan 97, Dec 97 \\
\hline 175 & $\mathrm{P}$ & 99 & 14.1 & 2.41 & -0.55 & 3.526 & 0.56 & 0.0 & 9.19 & $\mathrm{~V}$ & $\leq 11.0$ & $\ldots$ & .. & Dec 97 \\
\hline 176 & $\mathrm{C}$ & 99 & 11.6 & 1.89 & 0.47 & 3.591 & 0.54 & 0.8 & $\ldots$. & $\ldots$ & $\leq 11.0$ & $\ldots$ & .. & Dec 98 \\
\hline 180 & $\mathrm{C}$ & 99 & 14.4 & 2.94 & -0.54 & 3.494 & 0.05 & 0.2 & $\ldots$ & $\ldots$ & $41.9 \pm 9.6$ & $\ldots$ & .. & Dec 98 \\
\hline 187 & $\mathrm{C}$ & 99 & 14.3 & 2.85 & 0.22 & 3.643 & 0.53 & 1.4 & 14.41 & $\mathrm{E}$ & $\leq 11.0$ & $\ldots$ & .. & Dec 98 \\
\hline 191 & $\mathrm{P}$ & 99 & 14.2 & 2.78 & -0.37 & 3.535 & 0.18 & 0.0 & 8.63 & $\mathrm{~V}$ & $\leq 11.0$ & $\ldots$ & .. & Dec 97 \\
\hline 203 & $\mathrm{C}$ & 98 & 13.8 & 2.48 & -0.11 & 3.580 & 0.63 & 1.0 & $\ldots$. & $\ldots$ & $\leq 11.0$ & $\ldots$ & .. & Dec 97 \\
\hline 208 & $\mathrm{C}$ & 96 & 14.3 & 2.52 & -0.56 & 3.535 & 0.24 & 0.0 & $\ldots$ & $\ldots$ & $\leq 11.0$ & $\ldots$ & .. & Dec 98 \\
\hline 211 & $\mathrm{C}$ & 99 & 14.0 & 2.61 & -0.42 & 3.535 & 0.24 & 1.4 & 5.46 & $\mathrm{E}$ & $13.2 \pm 2.3$ & 1.42 & .. & Dec 97 \\
\hline 220 & $\mathrm{P}$ & 99 & 12.7 & 2.25 & 0.07 & 3.555 & 0.67 & 1.0 & 2.33 & $\mathrm{~V}$ & $19.7 \pm 3.1$ & 0.91 & .. & Jan 97 \\
\hline 221 & $\mathrm{C}$ & 98 & 11.3 & 2.66 & 1.41 & 3.679 & -0.01 & 1.5 & $\ldots$ & $\ldots$ & $56.9 \pm 5.3$ & $\ldots$ & .. & Jan 97 \\
\hline 222 & $\mathrm{P}$ & 98 & 14.1 & 2.85 & -0.23 & 3.544 & 0.71 & 1.2 & 5.17 & $\mathrm{~V}$ & $14.8 \pm 3.0$ & 1.51 & .. & Dec 97 \\
\hline 225 & $\mathrm{C}$ & 99 & 13.4 & 2.30 & -0.18 & 3.555 & 0.14 & 0.5 & $\ldots$ & $\ldots$ & $25.8 \pm 3.5$ & $\ldots$. & .. & Jan 97 \\
\hline 229 & $\mathrm{C}$ & 99 & 14.2 & 2.41 & -0.43 & 3.555 & 0.37 & 2.1 & 7.75 & $\mathrm{E}$ & $12.9 \pm 1.8$ & 1.98 & .. & Dec 97 \\
\hline 232 & $\mathrm{P}$ & 79 & 11.7 & 1.86 & 0.82 & 3.695 & 0.23 & $\ldots$. & 5.08 & $\mathrm{~V}$ & $34.0 \pm 2.2$ & 3.41 & .. & Dec 97 \\
\hline 237 & $\mathrm{C}$ & 99 & 15.3 & 3.22 & -0.78 & 3.509 & 0.75 & 0.0 & 5.22 & $\mathrm{E}$ & $\leq 11.0$ & $\ldots$ & .. & Dec 98 \\
\hline 239 & $\mathrm{P}$ & 99 & 12.7 & 2.25 & 0.05 & 3.555 & 0.38 & 1.5 & 4.45 & $\mathrm{~V}$ & $23.5 \pm 3.5$ & 2.07 & .. & Jan 97 \\
\hline 243 & $\mathrm{P}$ & 99 & 13.6 & 2.36 & -0.31 & 3.544 & 0.64 & 1.2 & 10.20 & $\mathrm{~V}$ & $12.5 \pm 1.8$ & 2.52 & .. & Dec 97 \\
\hline 248 & $\mathrm{P}$ & 99 & 12.7 & 2.17 & 0.10 & 3.571 & 0.59 & -0.9 & 6.86 & $\mathrm{~V}$ & $12.7 \pm 1.6$ & 1.72 & .. & Jan 97 \\
\hline 249 & $\mathrm{C}$ & 99 & 13.6 & 2.35 & 0.35 & 3.695 & 1.14 & 1.7 & $\ldots$ & $\ldots$ & $25.9 \pm 1.7$ & $\ldots$ & .. & Dec 97 \\
\hline 250 & $\mathrm{P}$ & 99 & 14.0 & 3.00 & -0.24 & 3.526 & 0.27 & 0.9 & 2.71 & $\mathrm{~V}$ & $20.5 \pm 2.7$ & 1.10 & .. & Dec 97 \\
\hline 254 & $\mathrm{P}$ & 99 & 14.3 & 3.21 & -0.24 & 3.526 & 0.50 & 5.6 & 3.52 & $\mathrm{~V}$ & $19.4 \pm 3.2$ & 1.35 & .. & Dec 98 \\
\hline 256 & $\mathrm{C}$ & 99 & 14.1 & 1.13 & -0.52 & 3.518 & $\ldots$ & 2.1 & $\ldots$ & $\ldots$ & $\leq 11.0$ & $\ldots$ & .. & Dec 98 \\
\hline 272 & $\mathrm{C}$ & 99 & 14.1 & 2.30 & -0.46 & 3.555 & 0.72 & -2.2 & $\ldots$ & $\ldots$ & $\leq 11.0$ & $\ldots$ & .. & Dec 98 \\
\hline 275 & $\mathrm{P}$ & 99 & 13.0 & 2.28 & -0.13 & 3.544 & 0.76 & 1.8 & 20.10 & $\mathrm{~V}$ & $77.6 \pm 15.7$ & $\ldots$ & .. & Dec 98 \\
\hline 284 & $\mathrm{P}$ & 99 & 14.6 & 3.11 & -0.41 & 3.526 & 1.18 & -2.0 & 3.11 & EV & $\ldots \ldots \ldots$ & $\ldots$ & .. & Jan 97 \\
\hline 291 & $\mathrm{C}$ & 99 & 12.9 & 2.07 & -0.06 & 3.562 & 0.32 & 1.5 & 1.91 & $\mathrm{E}$ & $14.3 \pm 1.9$ & 0.54 & .. & Dec 98 \\
\hline 305 & $\mathrm{C}$ & 99 & 14.6 & 2.39 & -0.72 & 3.518 & 0.42 & 0.0 & $\ldots$ & $\ldots$ & $13.8 \pm 2.5$ & $\ldots$ & .. & Dec 98 \\
\hline 308 & $\mathrm{P}$ & 0 & 12.6 & 2.54 & 0.06 & 3.526 & $\ldots$ & 0.7 & 4.17 & $\mathrm{~V}$ & $30.2 \pm 4.8$ & 2.49 & .. & Dec 98 \\
\hline 310 & $\mathrm{C}$ & 98 & 13.8 & 3.57 & -0.05 & 3.500 & 0.58 & 0.8 & $\ldots$. & $\ldots$ & $54.5 \pm 15.2$ & $\ldots$ & .. & Dec 98 \\
\hline 311 & $\mathrm{P}$ & 99 & 14.9 & 3.13 & -0.60 & 3.518 & 0.22 & 0.0 & 6.14 & $\mathrm{~V}$ & $\leq 11.0$ & $\ldots$. & .. & Dec 98 \\
\hline 315 & $\mathrm{P}$ & 99 & 14.8 & 2.85 & -0.73 & 3.518 & -0.03 & 0.0 & 8.85 & $\mathrm{~V}$ & $\leq 11.0$ & $\ldots$ & .. & Dec 97 \\
\hline 320 & $\mathrm{P}$ & 99 & 14.7 & 2.37 & -0.08 & 3.695 & 0.98 & 0.3 & 5.41 & $\mathrm{~V}$ & $11 . \overline{6} \pm 2.0$ & 1.24 & .. & Dec 97 \\
\hline 321 & $\mathrm{C}$ & 95 & 13.5 & 2.09 & -0.19 & 3.580 & $\ldots$ & -5.1 & 7.44 & $\mathrm{E}$ & $13.8 \pm 1.3$ & 2.03 & .. & Dec 97 \\
\hline 328 & $\mathrm{C}$ & 99 & 12.9 & 1.64 & 0.09 & 3.661 & 1.02 & 0.0 & 18.83 & $\mathrm{E}$ & $14.3 \pm 1.2$ & 5.32 & .. & Dec 97 \\
\hline 330 & $\mathrm{P}$ & 99 & 11.8 & 1.42 & 0.45 & 3.679 & 0.08 & 1.8 & 1.57 & $\mathrm{~V}$ & $25.5 \pm 1.3$ & 0.79 & .. & Jan 97 \\
\hline 332 & $\mathrm{C}$ & 99 & 14.2 & 2.07 & -0.59 & 3.518 & 2.46 & -0.9 & $\ldots$ & $\ldots$ & $\leq 11.0$ & $\ldots$ & .. & Dec 98 \\
\hline 333 & $\mathrm{C}$ & 99 & 14.7 & 1.92 & -0.56 & 3.483 & 0.54 & 3.7 & $\ldots$ & $\ldots$ & $\leq 11.0$ & $\ldots$ & .. & Dec 98 \\
\hline 334 & $\mathrm{P}$ & 99 & 13.6 & 2.35 & -0.20 & 3.562 & 1.20 & -4.8 & 5.27 & V & $16.9 \pm 3.2$ & 1.76 & .. & Dec 97 \\
\hline
\end{tabular}


Table 1-Continued

\begin{tabular}{|c|c|c|c|c|c|c|c|c|c|c|c|c|c|c|}
\hline JW & Sam & $\begin{array}{c}\text { Mem } \\
(\%)\end{array}$ & $\begin{array}{c}I \\
(\mathrm{mag})\end{array}$ & $\begin{array}{c}V-I \\
(\mathrm{mag})\end{array}$ & $\begin{array}{c}\log (\mathrm{L}) \\
\left(\mathrm{L}_{\odot}\right)\end{array}$ & $\begin{array}{c}\log \left(T_{\text {eff }}\right) \\
(\mathrm{K})\end{array}$ & $\begin{array}{c}\Delta(I-K) \\
\quad(\mathrm{mag})\end{array}$ & $\begin{array}{c}W_{\lambda}(\mathrm{CaII}) \\
(\AA)\end{array}$ & $\begin{array}{c}\text { Per } \\
\text { (days) }\end{array}$ & $\mathrm{Src}$ & $\begin{array}{c}v \sin (i) \\
\left(\mathrm{km} \mathrm{s}^{-1}\right)\end{array}$ & $\begin{array}{c}R \sin (i) \\
\quad\left(\mathrm{R}_{\odot}\right)\end{array}$ & SB2? & Date Obs \\
\hline 335 & $\mathrm{C}$ & 99 & 15.6 & 1.31 & -1.21 & 3.562 & 3.10 & -26.0 & $\ldots$ & $\ldots$ & $\ldots \ldots \ldots$ & $\ldots$ & .. & Dec 98 \\
\hline 337 & $\mathrm{P}$ & 99 & 11.7 & 1.76 & 0.34 & 3.591 & 0.52 & 1.9 & 19.50 & V & $\leq 11.0$ & $\ldots$. & .. & Dec 97 \\
\hline 345 & $\mathrm{P}$ & 81 & 12.4 & 2.38 & 0.37 & 3.571 & 0.42 & 0.0 & 8.21 & $\mathrm{~V}$ & $32.5 \pm 4.9$ & 5.27 & .. & Dec 97 \\
\hline 347 & $\mathrm{P}$ & 99 & 12.5 & 1.75 & 0.09 & 3.602 & 0.81 & 0.0 & 7.33 & VS & $\leq 11.0$ & $\ldots$ & .. & Dec 97 \\
\hline 348 & $\mathrm{C}$ & 99 & 11.2 & 1.53 & 0.92 & 3.719 & 1.00 & $\ldots$ & $\ldots$ & $\ldots$ & $21.7 \pm 1.1$ & $\ldots$ & .. & Dec 98 \\
\hline 352 & $\mathrm{P}$ & 99 & 10.4 & 2.10 & 1.09 & 3.591 & $\ldots$ & 1.9 & 8.00 & $\mathrm{~V}$ & $14.3 \pm 0.8$ & 2.26 & .. & Jan 97 \\
\hline 362 & $\mathrm{P}$ & 99 & 13.9 & 3.23 & -0.29 & 3.500 & 0.38 & 0.0 & 2.73 & $\mathrm{~V}$ & $23.2 \pm 8.3$ & 1.25 & .. & Dec 97 \\
\hline 363 & $\mathrm{C}$ & 98 & 12.7 & 2.51 & 0.12 & 3.544 & 0.40 & 1.1 & $\ldots$ & $\ldots$ & $33.4 \pm 7.6$ & $\ldots$ & .. & Jan 97 \\
\hline 365 & $\mathrm{P}$ & 99 & 11.7 & 1.96 & 0.82 & 3.679 & 0.40 & $\ldots$ & 4.08 & V & $41.6 \pm 3.0$ & 3.35 & .. & Jan 97 \\
\hline 373 & $\mathrm{C}$ & 99 & 13.5 & 3.37 & 0.99 & 3.679 & 0.20 & 2.1 & 9.81 & $\mathrm{E}$ & $16.3 \pm 0.8$ & 3.16 & .. & Dec 98 \\
\hline 378 & $\mathrm{P}$ & 99 & 12.9 & 2.11 & 0.05 & 3.580 & 1.08 & 1.5 & 9.03 & $\mathrm{~V}$ & $\leq 11.0$ & $\ldots$ & .. & Dec 97 \\
\hline 379 & $\mathrm{P}$ & 99 & 15.2 & 3.87 & -0.58 & 3.483 & 0.07 & $\ldots$ & 11.30 & VS & $\leq 11.0$ & $\ldots$. & .. & Dec 97 \\
\hline 381 & $\mathrm{P}$ & 99 & 13.2 & 2.11 & -0.14 & 3.571 & 1.26 & 0.0 & 16.20 & $\mathrm{~V}$ & $\leq 11.0$ & $\ldots$ & .. & Dec 97 \\
\hline 388 & $\mathrm{P}$ & 99 & 13.6 & 2.62 & 0.22 & 3.602 & 0.06 & 1.2 & 9.08 & $\mathrm{~V}$ & $11.9 \pm 0.9$ & 2.13 & .. & Dec 97 \\
\hline 398 & $\mathrm{C}$ & 99 & 14.5 & 2.44 & -0.41 & 3.580 & 0.30 & 2.2 & $\ldots$ & $\ldots$ & $\leq 11.0$ & $\ldots$ & .. & Dec 98 \\
\hline 406 & $\mathrm{P}$ & 99 & 13.9 & 3.41 & 0.37 & 3.562 & 0.31 & 1.7 & 2.25 & $\mathrm{~V}$ & $30.1 \pm 6.6$ & 1.34 & .. & Dec 97 \\
\hline 417 & $\mathrm{P}$ & 98 & 13.9 & 2.44 & -0.22 & 3.571 & 0.56 & 0.0 & 7.41 & V & $\leq 11.0$ & $\ldots$. & .. & Dec 97 \\
\hline 421 & $\mathrm{C}$ & 99 & 11.5 & 1.46 & 0.47 & 3.643 & 0.80 & -3.5 & $\ldots$ & $\ldots$ & $\leq 11.0$ & $\ldots$. & .. & Dec 98 \\
\hline 429 & $\mathrm{C}$ & 99 & 12.7 & 1.75 & 0.57 & 3.753 & $\ldots$ & 4.5 & $\ldots$ & $\ldots$ & $13.2 \pm 0.7$ & $\ldots$. & .. & Dec 98 \\
\hline 433 & $\mathrm{C}$ & 93 & 11.9 & 1.59 & 0.43 & 3.661 & 0.21 & $\ldots$ & $\ldots$ & $\ldots$ & $\leq 11.0$ & $\ldots$. & .. & Jan 97, Dec 97 \\
\hline 437 & $\mathrm{P}$ & 99 & 11.8 & 2.07 & 0.56 & 3.602 & 0.53 & 1.4 & 2.34 & V & $45.7 \pm 4.3$ & 2.11 & .. & Jan 97 \\
\hline 439 & $\mathrm{P}$ & 99 & 15.0 & 3.33 & -0.12 & 3.562 & 0.62 & 0.7 & 8.30 & V & $\leq 11.0$ & $\ldots$. & .. & Dec 98 \\
\hline 440 & $\mathrm{C}$ & 99 & 14.5 & 3.09 & -0.44 & 3.518 & -0.35 & 0.0 & $\ldots$ & $\ldots$ & $49.3 \pm 8.3$ & $\ldots$ & Y & Dec 98 \\
\hline 443 & $\mathrm{C}$ & 99 & 14.0 & 0.95 & -0.55 & 3.544 & 0.84 & -1.5 & $\ldots$ & $\ldots$ & $11.5 \pm 2.2$ & $\ldots$. & .. & Dec 98 \\
\hline 454 & $\mathrm{P}$ & 99 & 11.9 & 2.10 & 0.78 & 3.661 & 0.39 & -1.0 & 8.67 & V & $20.5 \pm 1.2$ & 3.51 & .. & Dec 97 \\
\hline 460 & $\mathrm{C}$ & 95 & 11.7 & 1.58 & 0.65 & 3.695 & 0.88 & $\ldots$ & $\ldots$ & $\ldots$ & $40.9 \pm 4.2$ & $\ldots$ & .. & Dec 98 \\
\hline 466 & $\mathrm{P}$ & 99 & 14.4 & 2.88 & -0.56 & 3.518 & 0.35 & $\ldots$ & 6.17 & V & $12.7 \pm 3.0$ & 1.55 & .. & Dec 97 \\
\hline 470 & $\mathrm{P}$ & 99 & 12.5 & 2.63 & 0.84 & 3.661 & $\ldots$ & 6.1 & 10.70 & V & $23.9 \pm 2.0$ & 5.05 & .. & Dec 98 \\
\hline 478 & $\mathrm{P}$ & 99 & 11.6 & 2.28 & 0.69 & 3.580 & 0.43 & $\ldots$ & 5.74 & V & $35.3 \pm 3.5$ & 4.00 & .. & Dec 97 \\
\hline 479 & $\mathrm{P}$ & 99 & 11.0 & 1.80 & 1.16 & 3.719 & 0.57 & 2.0 & 8.71 & V & $25.4 \pm 1.4$ & 4.37 & .. & Dec 98 \\
\hline 481 & $\mathrm{P}$ & 99 & 14.7 & 2.99 & -0.52 & 3.526 & 0.35 & 0.0 & 6.56 & V & ......... & $\ldots$ & .. & Dec 97 \\
\hline 482 & $\mathrm{C}$ & 99 & 13.3 & 2.38 & 0.10 & 3.591 & 0.60 & -50.3 & $\ldots$ & $\ldots$ & $13.9 \pm 3.0$ & $\ldots$ & .. & Dec 98 \\
\hline 485 & $\mathrm{P}$ & 99 & 14.3 & 2.49 & -0.42 & 3.555 & 1.18 & 0.8 & 9.66 & $\mathrm{~V}$ & $\leq 11.0$ & $\ldots$ & .. & Dec 97 \\
\hline 487 & $\mathrm{C}$ & 99 & 12.8 & 1.78 & 0.07 & 3.623 & 0.28 & 1.8 & $\ldots$ & $\ldots$ & $12.5 \pm 0.9$ & $\ldots$ & .. & Jan 97 \\
\hline 498 & $\mathrm{P}$ & 99 & 13.8 & 2.08 & -0.44 & 3.562 & $\ldots$ & $\ldots$ & 7.42 & $\mathrm{~V}$ & $11.7 \pm 1.4$ & 1.72 & .. & Dec 97 \\
\hline 501 & $\mathrm{C}$ & 99 & 13.2 & 1.82 & -0.28 & 3.580 & 0.62 & 1.1 & $\ldots$ & $\ldots$ & $\leq 11.0$ & $\ldots$ & .. & Dec 98 \\
\hline 502 & $\mathrm{C}$ & 99 & 13.5 & 2.57 & -0.22 & 3.535 & 0.97 & 0.0 & $\ldots$ & $\ldots$ & $23.8 \pm 4.7$ & $\ldots$ & .. & Dec 98 \\
\hline 526 & $\mathrm{P}$ & 98 & 11.9 & 1.90 & 0.52 & 3.623 & 0.74 & 1.2 & 1.68 & V & $95.3 \pm 14.6$ & 3.16 & .. & Jan 97 \\
\hline 527 & $\mathrm{C}$ & 99 & 13.0 & 2.09 & -0.06 & 3.571 & 0.63 & 0.8 & $\ldots$. & $\ldots$ & $12.7 \pm 1.5$ & $\ldots$. & .. & Dec 98 \\
\hline 534 & $\mathrm{C}$ & 85 & 12.7 & 1.43 & -0.03 & 3.544 & 1.33 & -69.5 & $\ldots$ & $\ldots$ & $\ldots \ldots$. & $\ldots$ & .. & Dec 98 \\
\hline 544 & $\mathrm{P}$ & 99 & 10.8 & 2.22 & 1.30 & 3.670 & -0.17 & 1.6 & 3.42 & $\mathrm{~V}$ & $58.8 \pm 5.8$ & 3.97 & .. & Jan 97 \\
\hline 565 & $\mathrm{C}$ & 99 & 14.5 & 2.95 & -0.62 & 3.509 & 0.22 & 0.0 & $\ldots$ & $\ldots$ & $\leq 11.0$ & $\ldots$. & .. & Dec 98 \\
\hline 567 & $\mathrm{P}$ & 99 & 9.7 & 1.73 & 1.43 & 3.661 & -0.17 & -1.7 & 8.53 & $\mathrm{~V}$ & $22.2 \pm 1.1$ & 3.74 & .. & Dec 97 \\
\hline 576 & $\mathrm{P}$ & 99 & 13.1 & 2.28 & -0.07 & 3.555 & 0.23 & 0.0 & 2.01 & $\mathrm{~V}$ & $30.5 \pm 4.5$ & 1.21 & .. & Dec 97 \\
\hline 585 & $\mathrm{C}$ & 99 & 11.5 & 1.37 & 0.39 & 3.602 & $\ldots$ & 1.8 & $\ldots$ & $\ldots$ & $18.4 \pm 0.9$ & $\ldots$ & .. & Dec 98 \\
\hline 589 & $\mathrm{P}$ & 99 & 11.3 & 2.12 & 1.23 & 3.719 & 0.11 & -0.6 & 14.30 & $\mathrm{~V}$ & $14.6 \pm 0.7$ & 4.12 & .. & Dec 98 \\
\hline 592 & $\mathrm{C}$ & 99 & 14.4 & 2.58 & -0.57 & 3.535 & 0.06 & 0.0 & 7.92 & $\mathrm{E}$ & $\leq 11.0$ & $\ldots$ & .. & Dec 98 \\
\hline 601 & $\mathrm{P}$ & 98 & 12.5 & 1.25 & 0.05 & 3.679 & 0.05 & 2.5 & 2.22 & V & $11.9 \pm 0.5$ & 0.52 & .. & Jan 97 \\
\hline
\end{tabular}


Table 1-Continued

\begin{tabular}{|c|c|c|c|c|c|c|c|c|c|c|c|c|c|c|}
\hline JW & Sam & $\begin{array}{c}\text { Mem } \\
(\%)\end{array}$ & $\begin{array}{c}I \\
(\mathrm{mag})\end{array}$ & $\begin{array}{c}V-I \\
(\mathrm{mag})\end{array}$ & $\begin{array}{c}\log (\mathrm{L}) \\
\left(\mathrm{L}_{\odot}\right)\end{array}$ & $\begin{array}{c}\log \left(\mathrm{T}_{\text {eff }}\right) \\
(\mathrm{K})\end{array}$ & $\begin{array}{c}\Delta(I-K) \\
\quad(\mathrm{mag})\end{array}$ & $\begin{array}{c}W_{\lambda}(\mathrm{CaII}) \\
(\AA)\end{array}$ & $\begin{array}{l}\text { Per } \\
\text { (days) }\end{array}$ & Src & $\begin{array}{c}v \sin (i) \\
\left(\mathrm{km} \mathrm{s}^{-1}\right)\end{array}$ & $\begin{array}{c}R \sin (i) \\
\left(\mathrm{R}_{\odot}\right)\end{array}$ & SB2? & Date Obs \\
\hline 625 & $\mathrm{C}$ & 99 & 14.4 & 1.07 & -0.73 & 3.562 & 1.37 & 6.1 & $\cdots$ & $\ldots$ & $\ldots \ldots \ldots$ & $\ldots$ & .. & Dec 98 \\
\hline 629 & $\mathrm{C}$ & 99 & 13.5 & 4.34 & 1.47 & 3.643 & 1.41 & -16.9 & $\ldots$ & $\ldots$ & $56.3 \pm 11.3$ & $\ldots$ & .. & Dec 98 \\
\hline 631 & $\mathrm{C}$ & 99 & 12.9 & 2.20 & 0.22 & 3.602 & 1.29 & 1.6 & 0.96 & $\mathrm{E}$ & $39.7 \pm 3.7$ & 0.75 & .. & Dec 98 \\
\hline 636 & $\mathrm{P}$ & 99 & 13.4 & 2.46 & 0.04 & 3.575 & 0.79 & $\ldots$ & 5.59 & $\mathrm{~V}$ & $\leq 11.0$ & $\ldots$ & .. & Jan 97 \\
\hline 639 & $\mathrm{P}$ & 99 & 13.6 & 2.53 & -0.37 & 3.526 & $\ldots$ & $\ldots$ & 5.24 & $\mathrm{~V}$ & $12.7 \pm 1.7$ & 1.31 & .. & Dec 97 \\
\hline 641 & $\mathrm{P}$ & 99 & 11.5 & 1.74 & 0.91 & 3.719 & 0.12 & 0.0 & 3.17 & $\mathrm{~V}$ & $46.8 \pm 3.8$ & 2.93 & .. & Jan 97 \\
\hline 647 & C & 99 & 13.7 & 2.31 & -0.22 & 3.494 & $\ldots$ & -5.0 & & $\ldots$ & $13.8 \pm 1.3$ & $\ldots$ & .. & Dec 98 \\
\hline 648 & $\mathrm{P}$ & 99 & 13.2 & 2.58 & 0.24 & 3.580 & $\ldots$ & 0.9 & 34.50 & $\mathrm{~V}$ & $\leq 11.0$ & $\ldots$ & .. & Dec 98 \\
\hline 661 & $\mathrm{C}$ & 99 & 13.5 & 1.57 & -0.39 & 3.580 & 1.80 & 2.1 & $\ldots$ & $\ldots$ & $\leq 11.0$ & $\ldots$ & .. & Dec 97 \\
\hline 662 & $\mathrm{C}$ & 95 & 14.4 & 1.78 & -0.57 & 3.623 & 1.36 & -1.0 & $\ldots$ & $\ldots$ & $30.7 \pm 4.8$ & $\ldots$ & .. & Dec 98 \\
\hline 664 & $\mathrm{P}$ & 99 & 14.1 & 2.88 & 0.26 & 3.623 & -0.18 & 1.4 & 7.20 & $\mathrm{~V}$ & $\leq 11.0$ & $\ldots$ & .. & Dec 97 \\
\hline 669 & $\mathrm{P}$ & 99 & 10.8 & 1.67 & 1.12 & 3.708 & 0.14 & 1.8 & 1.81 & $\mathrm{~V}$ & $58.4 \pm 4.0$ & 2.09 & Y & Jan 97, Dec 97 \\
\hline 673 & $\mathrm{P}$ & 99 & 13.1 & 1.83 & 0.06 & 3.643 & 0.81 & 1.3 & 1.44 & $\mathrm{EV}$ & $18.7 \pm 1.3$ & 0.53 & .. & Jan 97 \\
\hline 678 & $\mathrm{P}$ & 99 & 12.3 & 1.84 & 0.55 & 3.695 & 0.24 & $\ldots$ & 13.00 & $\mathrm{~V}$ & $12.4 \pm 0.7$ & 3.18 & .. & Dec 97 \\
\hline 683 & $\mathrm{P}$ & 99 & 11.8 & 1.85 & 0.53 & 3.623 & 0.28 & 1.9 & 11.50 & $\mathrm{~V}$ & $\leq 11.0$ & $\ldots$. & .. & Dec 98 \\
\hline 694 & $\mathrm{C}$ & 99 & 14.7 & 2.99 & 0.05 & 3.623 & 0.18 & -3.8 & $\ldots$. & $\ldots$ & $\leq 11.0$ & $\ldots$ & .. & Dec 98 \\
\hline 701 & $\mathrm{C}$ & 99 & 12.4 & 1.26 & 0.25 & 3.722 & 1.27 & -8.3 & $\ldots$ & $\ldots$ & $12.4 \pm 0.8$ & $\ldots$. & .. & Dec 98 \\
\hline 704 & $\mathrm{P}$ & 99 & 14.7 & 2.41 & -0.59 & 3.562 & $\ldots$ & $\ldots$ & 6.87 & $\mathrm{~V}$ & $12.7 \pm 3.7$ & 1.72 & .. & Dec 97 \\
\hline 706 & $\mathrm{C}$ & 99 & 11.8 & 1.34 & 0.39 & 3.679 & -0.03 & $\ldots$ & $\ldots$ & $\ldots$ & $12.0 \pm 0.6$ & $\ldots$. & .. & Dec 98 \\
\hline 710 & $\mathrm{P}$ & 99 & 12.7 & 1.17 & -0.04 & 3.562 & 0.68 & -2.1 & 7.59 & $\mathrm{~V}$ & $\leq 11.0$ & $\ldots$ & .. & Dec 98 \\
\hline 716 & $\mathrm{P}$ & 99 & 14.4 & 2.88 & -0.57 & 3.500 & 1.15 & -2.7 & 3.95 & $\mathrm{~V}$ & $\leq 11.0$ & $\ldots$ & .. & Dec 98 \\
\hline 717 & $\mathrm{P}$ & 99 & 16.1 & $\ldots$ & -1.39 & 3.535 & $\ldots$ & 0.0 & 4.00 & $\mathrm{~V}$ & $\ldots \ldots \ldots$ & $\ldots$. & .. & Dec 98 \\
\hline 719 & $\mathrm{C}$ & 99 & 14.5 & 3.06 & -0.56 & 3.494 & 0.10 & 0.0 & 6.88 & $\mathrm{E}$ & $\leq 11.0$ & $\ldots$ & .. & Dec 97 \\
\hline 721 & $\mathrm{P}$ & 99 & 13.1 & 2.08 & -0.06 & 3.580 & 0.82 & -3.8 & 2.45 & $\mathrm{~V}$ & $\leq 11.0$ & $\ldots$. & .. & Dec 97 \\
\hline 727 & $\mathrm{P}$ & 99 & 13.9 & 2.44 & -0.38 & 3.544 & 0.75 & 0.8 & 6.03 & $\mathrm{~V}$ & $13.3 \pm 2.7$ & 1.58 & .. & Dec 97 \\
\hline 728 & $\mathrm{C}$ & 99 & 13.6 & 2.32 & -0.20 & 3.562 & 0.54 & 0.9 & 13.87 & $\mathrm{E}$ & $\leq 11.0$ & $\ldots$ & .. & Dec 97 \\
\hline 731 & $\mathrm{P}$ & 88 & 12.3 & 1.74 & 0.10 & 3.591 & 0.28 & 2.2 & 7.64 & $\mathrm{~V}$ & $12.7 \pm 0.9$ & 1.92 & .. & Dec 97 \\
\hline 733 & $\mathrm{C}$ & 99 & 13.1 & 2.33 & 0.04 & 3.571 & 0.58 & 1.7 & 3.28 & $\mathrm{E}$ & $20.8 \pm 3.0$ & 1.35 & .. & Dec 98 \\
\hline 736 & $\mathrm{C}$ & 99 & 13.9 & 2.27 & -0.25 & 3.580 & 0.65 & 1.6 & 11.68 & $\mathrm{E}$ & $\leq 11.0$ & $\ldots$ & .. & Dec 98 \\
\hline 744 & $\mathrm{C}$ & 99 & 13.4 & 1.75 & -0.36 & 3.562 & 2.42 & -0.9 & $\ldots$ & $\ldots$ & $\leq 11.0$ & $\ldots$ & .. & Dec 98 \\
\hline 751 & $\mathrm{C}$ & 99 & 13.5 & 2.47 & -0.07 & 3.562 & 0.78 & -3.0 & $\ldots$ & $\ldots$ & $\leq 11.0$ & $\ldots$ & .. & Dec 97 \\
\hline 756 & $\mathrm{C}$ & 99 & 12.8 & 2.15 & 0.09 & 3.580 & 1.67 & -2.0 & $\ldots$ & $\ldots$ & $33.2 \pm 2.7$ & $\ldots$ & .. & Dec 98 \\
\hline 758 & $\mathrm{P}$ & 99 & 14.1 & 3.45 & -0.15 & 3.509 & 0.48 & -2.0 & 2.51 & $\mathrm{~V}$ & $16.0 \pm 3.0$ & 0.79 & .. & Dec 98 \\
\hline 765 & $\mathrm{P}$ & 97 & 13.2 & 2.67 & -0.18 & 3.522 & 0.09 & 1.3 & 2.42 & $\mathrm{~V}$ & $14.2 \pm 2.4$ & 0.68 & .. & Dec 97 \\
\hline 779 & $\mathrm{C}$ & 95 & 12.5 & 1.34 & 0.05 & 3.661 & 0.55 & $\ldots$ & $\ldots$ & $\ldots$ & $18.7 \pm 1.1$ & $\ldots$. & .. & Dec 98 \\
\hline 782 & $\mathrm{C}$ & 99 & 14.2 & 2.14 & -0.41 & 3.591 & 2.18 & -2.5 & $\cdots$ & $\ldots$ & $\leq 11.0$ & $\ldots$ & .. & Dec 98 \\
\hline 786 & $\mathrm{P}$ & 89 & 13.9 & 2.39 & -0.23 & 3.571 & 1.30 & 0.0 & 8.81 & $\mathrm{~V}$ & $\leq 11.0$ & $\ldots$ & .. & Dec 97 \\
\hline 787 & $\mathrm{P}$ & 99 & 12.7 & $\ldots$ & -0.07 & 3.571 & $\ldots$ & 0.6 & 9.09 & $\mathrm{E}$ & $\leq 11.0$ & $\ldots$ & .. & Dec 98 \\
\hline 788 & $\mathrm{P}$ & 99 & 14.5 & 3.48 & -0.28 & 3.509 & 0.28 & 0.0 & 4.82 & $\mathrm{EV}$ & $\leq 11.0$ & $\ldots$ & .. & Dec 97 \\
\hline 790 & $\mathrm{P}$ & 79 & 10.9 & 1.06 & 0.73 & 3.719 & 0.12 & 2.2 & 2.74 & $\mathrm{~V}$ & $39.8 \pm 2.8$ & 2.15 & .. & Jan 97 \\
\hline 792 & $\mathrm{P}$ & 99 & 13.6 & 2.64 & 0.08 & 3.580 & 1.05 & 1.4 & 10.30 & $\mathrm{~V}$ & $\leq 11.0$ & $\ldots$ & .. & Dec 97 \\
\hline 794 & $\mathrm{P}$ & 0 & 10.9 & 0.81 & 0.62 & 3.722 & 0.03 & $\ldots$ & 2.62 & $\mathrm{~V}$ & $\leq 11.0$ & $\ldots$ & .. & Dec 98 \\
\hline 795 & $\mathrm{P}$ & 99 & 10.6 & 1.19 & 1.02 & 3.744 & 0.12 & 1.7 & 1.55 & V & $60.9 \pm 5.4$ & 1.86 & .. & Jan 97 \\
\hline 811 & $\mathrm{P}$ & 99 & 13.4 & 2.56 & 0.13 & 3.580 & 1.25 & 0.0 & 11.10 & $\mathrm{~V}$ & $\leq 11.0$ & $\ldots$ & .. & Dec 98 \\
\hline 813 & $\mathrm{P}$ & 99 & 12.4 & 1.75 & 0.05 & 3.580 & 0.56 & 1.9 & 2.85 & $\mathrm{EV}$ & $26.0 \pm 2.5$ & 1.46 & .. & Dec 97 \\
\hline 815 & $\mathrm{P}$ & 99 & 13.8 & 2.69 & -0.34 & 3.526 & 0.52 & 0.0 & 6.42 & $\mathrm{~V}$ & $12.9 \pm 1.9$ & 1.64 & .. & Dec 97 \\
\hline 817 & $\mathrm{P}$ & 99 & 13.8 & 3.04 & -0.30 & 3.500 & 0.22 & $\ldots$ & 2.23 & $\mathrm{EV}$ & $40.0 \pm 6.5$ & 1.76 & .. & Jan 97, Dec 97 \\
\hline 818 & $\mathrm{C}$ & 98 & 12.1 & 1.82 & 0.20 & 3.591 & 0.26 & $\ldots$ & $\ldots$ & $\ldots$ & $14.5 \pm 0.9$ & $\ldots$ & .. & Dec 98 \\
\hline
\end{tabular}


Table 1-Continued

\begin{tabular}{|c|c|c|c|c|c|c|c|c|c|c|c|c|c|c|}
\hline JW & Sam & $\begin{array}{c}\text { Mem } \\
(\%)\end{array}$ & $\begin{array}{c}I \\
(\mathrm{mag})\end{array}$ & $\begin{array}{c}V-I \\
(\mathrm{mag})\end{array}$ & $\begin{array}{c}\log (\mathrm{L}) \\
\left(\mathrm{L}_{\odot}\right)\end{array}$ & $\begin{array}{c}\log \left(\mathrm{T}_{\text {eff }}\right) \\
(\mathrm{K})\end{array}$ & $\begin{array}{c}\Delta(I-K) \\
\quad(\mathrm{mag})\end{array}$ & $\begin{array}{c}W_{\lambda}(\mathrm{CaII}) \\
(\AA)\end{array}$ & $\begin{array}{c}\text { Per } \\
\text { (days) }\end{array}$ & Src & $\begin{array}{c}v \sin (i) \\
\left(\mathrm{km} \mathrm{s}^{-1}\right)\end{array}$ & $\begin{array}{c}R \sin (i) \\
\left(\mathrm{R}_{\odot}\right)\end{array}$ & SB2? & Date Obs \\
\hline 819 & $\mathrm{P}$ & 99 & 13.2 & 2.85 & -0.06 & 3.518 & 0.12 & 1.3 & 1.21 & $\mathrm{E}$ & $27.7 \pm 9.4$ & 0.66 & .. & Dec 97 \\
\hline 823 & $\mathrm{P}$ & 99 & 13.9 & 3.28 & -0.31 & 3.494 & 0.25 & 0.0 & 9.00 & $\mathrm{~V}$ & $\leq 11.0$ & $\ldots$ & .. & Dec 98 \\
\hline 826 & $\mathrm{P}$ & 91 & 14.0 & 1.38 & -0.60 & 3.602 & 3.01 & -6.3 & 9.57 & $\mathrm{~V}$ & $\leq 11.0$ & $\ldots$ & .. & Dec 97 \\
\hline 830 & $\mathrm{P}$ & 99 & 13.0 & 2.36 & 0.01 & 3.555 & 0.63 & 1.8 & 9.23 & $\mathrm{~V}$ & $\leq 11.0$ & $\ldots$ & .. & Dec 98 \\
\hline 834 & $\mathrm{C}$ & 99 & 13.0 & 2.24 & $\ldots$ & $\ldots$ & $\ldots$ & . & 7.01 & $\mathrm{E}$ & $13.4 \pm 1.2$ & 2.44 & .. & Dec 98 \\
\hline 835 & $\mathrm{P}$ & 99 & 14.1 & 2.52 & -0.54 & 3.526 & 0.24 & 0.0 & 8.85 & $\mathrm{~V}$ & $\leq 11.0$ & $\ldots$ & .. & Dec 97 \\
\hline 836 & $\mathrm{P}$ & 99 & 13.4 & 2.20 & -0.11 & 3.580 & 1.10 & 0.9 & 12.20 & V & $\leq 11.0$ & $\ldots$ & .. & Dec 97 \\
\hline 837 & $\mathrm{P}$ & 99 & 13.3 & 2.21 & -0.06 & 3.580 & 0.62 & 1.2 & 10.60 & $\mathrm{~V}$ & $\leq 11.0$ & $\ldots$ & .. & Dec 97 \\
\hline 839 & $\mathrm{P}$ & 99 & 13.5 & 3.70 & 0.70 & 3.562 & -1.41 & 1.2 & 7.52 & $\mathrm{~V}$ & $\leq 11.0$ & $\ldots$ & .. & Dec 97 \\
\hline 843 & $\mathrm{P}$ & 98 & 13.8 & 2.45 & -0.33 & 3.544 & 0.02 & 0.0 & 0.84 & $\mathrm{E}$ & $89.5 \pm 16.3$ & 1.48 & .. & Jan 97,Dec 97 \\
\hline 850 & $\mathrm{P}$ & 99 & 13.4 & 2.14 & -0.11 & 3.591 & 0.73 & -4.1 & 7.78 & $\mathrm{~V}$ & $\leq 11.0$ & $\ldots$ & .. & Jan 97 \\
\hline 855 & $\mathrm{P}$ & 99 & 14.1 & 2.34 & -0.51 & 3.544 & 0.79 & 0.0 & 7.08 & $\mathrm{~V}$ & $11.8 \pm 2.0$ & 1.65 & .. & Dec 97 \\
\hline 862 & $\mathrm{C}$ & 99 & 15.5 & 3.80 & -0.77 & 3.471 & 0.24 & -4.5 & 3.49 & $\mathrm{E}$ & $\ldots \ldots \ldots$ & $\ldots$ & .. & Dec 97 \\
\hline 863 & $\mathrm{P}$ & 99 & 14.0 & 2.62 & -0.50 & 3.518 & 0.71 & 0.0 & 7.91 & V & $\leq 11.0$ & $\ldots$ & .. & Dec 97 \\
\hline 864 & $\mathrm{C}$ & 99 & 14.4 & 2.46 & -0.70 & 3.526 & 0.59 & 0.7 & 3.70 & $\mathrm{E}$ & $\ldots \ldots \ldots$ & & .. & Dec 98 \\
\hline 866 & $\mathrm{P}$ & 98 & 11.8 & 2.13 & 0.99 & 3.708 & 0.18 & $\ldots$ & 6.76 & $\mathrm{~V}$ & $27.4 \pm 1.6$ & 3.66 & .. & Jan 97 \\
\hline 868 & $\mathrm{C}$ & 99 & 13.2 & 1.96 & 0.16 & 3.661 & 0.42 & 1.4 & $\ldots$ & $\ldots$ & $13.7 \pm 0.8$ & $\ldots$ & .. & Dec 98 \\
\hline 872 & $\mathrm{P}$ & 99 & 15.2 & 3.24 & -0.83 & 3.494 & 0.60 & 0.4 & 4.19 & $\mathrm{~V}$ & $\ldots \ldots \ldots$ & $\ldots$ & .. & Dec 97 \\
\hline 880 & $\mathrm{P}$ & 99 & 14.2 & 2.74 & -0.51 & 3.509 & -0.10 & 0.0 & 1.58 & $\mathrm{E}$ & $19.4 \pm 1.9$ & 0.61 & .. & Dec 98 \\
\hline 883 & $\mathrm{C}$ & 99 & 13.3 & 2.62 & 0.12 & 3.562 & $\ldots$ & $\ldots$ & 0.85 & $\mathrm{E}$ & $61.3 \pm 15.3$ & 1.03 & $\mathrm{Y}$ & Jan 97 \\
\hline 887 & $\mathrm{C}$ & 97 & 10.3 & 1.42 & 1.39 & 3.771 & 0.05 & $\ldots$ & $\ldots$ & $\ldots$ & $66.2 \pm 6.3$ & $\ldots$ & .. & Dec 98 \\
\hline 890 & $\mathrm{P}$ & 99 & 13.0 & 2.11 & -0.16 & 3.555 & 0.46 & 1.6 & 1.10 & $\mathrm{~V}$ & $61.6 \pm 13.3$ & 1.34 & .. & Jan 97 \\
\hline 892 & $\mathrm{P}$ & 99 & 14.0 & 2.22 & -0.13 & 3.623 & $\ldots$ & 1.0 & 8.56 & V & $12.0 \pm 0.9$ & 2.03 & .. & Dec 97 \\
\hline 906 & $\mathrm{C}$ & 99 & 14.2 & 2.45 & -0.58 & 3.526 & 0.36 & 1.6 & 10.66 & $\mathrm{E}$ & $\leq 11.0$ & $\ldots$ & .. & Dec 97 \\
\hline 907 & $\mathrm{P}$ & 99 & 12.8 & 1.60 & 0.17 & 3.679 & -0.20 & 1.8 & 1.65 & $\mathrm{~V}$ & $37.3 \pm 2.8$ & 1.22 & .. & Jan 97 \\
\hline 911 & $\mathrm{C}$ & 99 & 12.7 & 1.62 & 0.15 & 3.661 & 1.01 & $\ldots$ & $\ldots$ & $\ldots$ & $\leq 11.0$ & $\ldots$ & .. & Dec 98 \\
\hline 912 & $\mathrm{C}$ & 99 & 13.9 & 2.54 & -0.48 & 3.526 & 0.48 & 1.2 & $\ldots$ & $\ldots$ & $\leq 11.0$ & $\ldots$ & .. & Dec 98 \\
\hline 914 & $\mathrm{P}$ & 99 & 13.2 & 2.09 & -0.23 & 3.555 & 0.71 & 0.0 & 7.71 & $\mathrm{~V}$ & $\leq 11.0$ & $\ldots$ & .. & Dec 97 \\
\hline 919 & $\mathrm{C}$ & 97 & 14.4 & 2.93 & -0.45 & 3.526 & -0.22 & 1.4 & $\ldots$ & $\ldots$ & $\leq 11.0$ & $\ldots$ & .. & Dec 98 \\
\hline 926 & $\mathrm{C}$ & 96 & 12.8 & 1.93 & 0.11 & 3.602 & 0.09 & 1.8 & 5.54 & $\mathrm{E}$ & $12 . \overline{3} \pm 1.0$ & 1.35 & .. & Dec 98 \\
\hline 927 & $\mathrm{C}$ & 99 & 14.0 & 2.29 & -0.43 & 3.509 & 0.01 & 1.0 & 2.02 & $\mathrm{E}$ & $22.4 \pm 5.7$ & 0.89 & .. & Dec 97 \\
\hline 930 & $\mathrm{P}$ & 99 & 14.1 & 2.56 & 0.04 & 3.623 & -0.71 & 5.2 & 2.88 & $\mathrm{~V}$ & $15.0 \pm 1.7$ & 0.85 & .. & Dec 97 \\
\hline 933 & $\mathrm{P}$ & 98 & 13.7 & 2.48 & -0.31 & 3.509 & 0.05 & 1.0 & 5.98 & $\mathrm{~V}$ & $\leq 11.0$ & $\ldots$ & .. & Jan 97 \\
\hline 938 & $\mathrm{C}$ & 99 & 12.6 & 2.70 & 0.99 & 3.695 & 0.19 & 1.3 & 5.15 & $\mathrm{E}$ & $21.7 \pm 2.6$ & 2.21 & .. & Dec 97 \\
\hline 940 & $\mathrm{C}$ & 95 & 12.0 & 1.63 & 0.25 & 3.535 & -0.13 & $\ldots$ & $\ldots$ & $\ldots$ & $61.6 \pm 14.9$ & $\ldots$ & .. & Dec 98 \\
\hline 945 & $\mathrm{C}$ & 99 & 12.5 & 2.40 & 2.29 & 4.111 & 0.58 & $\ldots$ & $\ldots$ & $\ldots$ & ......... & $\ldots$ & .. & Dec 98 \\
\hline 949 & $\mathrm{C}$ & 90 & 12.3 & 2.55 & 0.47 & 3.562 & 0.55 & 0.0 & $\ldots$ & $\ldots$ & $67.3 \pm 18.8$ & $\ldots$ & .. & Dec 97 \\
\hline 961 & $\mathrm{C}$ & 98 & 12.3 & 1.71 & 0.19 & 3.612 & 0.18 & 1.9 & 1.43 & $\mathrm{E}$ & $66.2 \pm 7.2$ & 1.87 & $\mathrm{Y}$ & Dec 98 \\
\hline 962 & $\mathrm{C}$ & 99 & 13.3 & 1.97 & 0.00 & 3.623 & 0.80 & 1.8 & 9.56 & $\mathrm{E}$ & $\leq 11.0$ & $\ldots$ & .. & Dec 98 \\
\hline 969 & $\mathrm{C}$ & 99 & 14.4 & 2.54 & -0.54 & 3.544 & 0.58 & -2.7 & $\ldots$ & $\ldots$ & $14 . \overline{0} \pm 1.8$ & $\ldots$ & .. & Dec 98 \\
\hline 971 & $\mathrm{C}$ & 83 & 11.5 & 1.51 & 0.40 & 3.602 & 0.19 & 2.4 & $\ldots$ & $\ldots$ & $42.7 \pm 3.6$ & $\ldots$ & .. & Dec 98 \\
\hline 972 & $\mathrm{P}$ & 99 & 13.3 & 2.02 & -0.28 & 3.562 & 0.38 & 1.2 & 8.07 & $\mathrm{~V}$ & $11.1 \pm 2.1$ & 1.77 & .. & Dec 97 \\
\hline 988 & $\mathrm{C}$ & 99 & 14.8 & 3.12 & -0.34 & 3.544 & 0.81 & -4.2 & $\ldots$ & $\ldots$ & $19.9 \pm 6.1$ & $\ldots$ & .. & Dec 97 \\
\hline 990 & $\mathrm{C}$ & 99 & 14.1 & 2.88 & -0.42 & 3.500 & -0.14 & 0.6 & 2.16 & $\mathrm{E}$ & $21.4 \pm 3.6$ & 0.91 & .. & Dec 98 \\
\hline 995 & $\mathrm{C}$ & 98 & 13.0 & 2.11 & -0.14 & 3.555 & 0.22 & 0.9 & $\ldots$ & $\ldots$ & $\leq 11.0$ & $\ldots$ & .. & Jan 97 \\
\hline 998 & $\mathrm{C}$ & 99 & 13.2 & 2.32 & -0.24 & 3.535 & 0.11 & $\ldots$ & $\ldots$ & $\ldots$ & $12.8 \pm 1.6$ & $\ldots$ & .. & Dec 98 \\
\hline 1002 & $\mathrm{C}$ & 99 & 14.6 & 3.08 & -0.41 & 3.471 & 0.51 & -18.6 & $\ldots$. & $\ldots$ & $\ldots \ldots \ldots$ & $\ldots$. & .. & Dec 98 \\
\hline 1004 & $\mathrm{C}$ & 99 & 13.8 & 2.10 & -0.40 & 3.562 & 0.31 & 2.0 & $\ldots$ & $\ldots$ & $\leq 11.0$ & $\ldots$ & .. & Dec 98 \\
\hline
\end{tabular}


Table 1-Continued

\begin{tabular}{|c|c|c|c|c|c|c|c|c|c|c|c|c|c|c|}
\hline JW & Sam & $\begin{array}{c}\text { Mem } \\
(\%)\end{array}$ & $\begin{array}{c}I \\
(\mathrm{mag})\end{array}$ & $\begin{array}{c}V-I \\
(\mathrm{mag})\end{array}$ & $\begin{array}{c}\log (\mathrm{L}) \\
\left(\mathrm{L}_{\odot}\right)\end{array}$ & $\begin{array}{c}\log \left(\mathrm{T}_{\text {eff }}\right) \\
(\mathrm{K})\end{array}$ & $\begin{array}{c}\Delta(I-K) \\
\quad(\mathrm{mag})\end{array}$ & $\begin{array}{c}W_{\lambda}(\mathrm{CaII}) \\
(\AA)\end{array}$ & $\begin{array}{c}\text { Per } \\
\text { (days) }\end{array}$ & $\mathrm{Src}$ & $\begin{array}{c}v \sin (i) \\
\left(\mathrm{km} \mathrm{s}^{-1}\right)\end{array}$ & $\begin{array}{c}R \sin (i) \\
\quad\left(\mathrm{R}_{\odot}\right)\end{array}$ & SB2? & Date Obs \\
\hline 1006 & $\mathrm{C}$ & 99 & 14.7 & 2.92 & -0.65 & 3.518 & 2.21 & 1.2 & $\ldots$ & $\ldots$ & $\leq 11.0$ & $\ldots$ & .. & Dec 98 \\
\hline 1007 & $\mathrm{C}$ & 99 & 14.5 & 2.50 & -0.72 & 3.526 & -0.00 & 1.1 & $\ldots$ & $\ldots$ & $11.1 \pm 1.5$ & $\ldots$ & .. & Dec 98 \\
\hline 1008 & $\mathrm{C}$ & 92 & 12.7 & 1.71 & -0.08 & 3.571 & 0.03 & 2.0 & 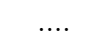 & $\ldots$ & $\leq 11.0$ & 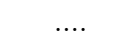 & .. & Jan 97 \\
\hline 1013 & $\mathrm{C}$ & 99 & 14.6 & 2.30 & -0.74 & 3.526 & 0.64 & -26.5 & $\ldots$ & $\ldots$ & $25.6 \pm 5.7$ & $\ldots$ & .. & Dec 98 \\
\hline 1016 & $\mathrm{C}$ & 88 & 13.0 & 1.92 & 0.16 & 3.643 & 0.38 & $\ldots$ & $\ldots$ & $\ldots$ & $12.4 \pm 1.0$ & $\ldots$ & .. & Dec 98 \\
\hline 1020 & $\mathrm{C}$ & 99 & 12.7 & 1.40 & -0.08 & 3.623 & -0.01 & 1.7 & $\ldots$ & $\ldots$ & $\leq 11.0$ & $\ldots$ & .. & Jan 97 \\
\hline 1021 & $\mathrm{C}$ & 36 & 13.0 & 1.91 & 0.11 & 3.633 & 0.34 & 1.6 & 7.75 & $\mathrm{E}$ & $15.0 \pm 1.7$ & 2.30 & .. & Dec 97 \\
\hline 1026 & $\mathrm{C}$ & 98 & 13.8 & 2.24 & -0.27 & 3.571 & 1.41 & -1.3 & 4.21 & $\mathrm{E}$ & $15.3 \pm 1.7$ & 1.28 & .. & Jan 97,Dec 97 \\
\hline 1030 & $\mathrm{C}$ & 99 & 13.7 & 2.18 & -0.43 & 3.544 & 0.36 & 1.5 & $\ldots$ & $\ldots$ & $11.9 \pm 1.4$ & $\ldots$ & .. & Dec 98 \\
\hline 1033 & $\mathrm{C}$ & 80 & 14.7 & 2.04 & 0.49 & 3.917 & -0.31 & 2.0 & $\ldots$ & $\ldots$ & $34.2 \pm 5.7$ & $\ldots$ & .. & Dec 98 \\
\hline 1035 & $\mathrm{C}$ & 98 & 14.1 & 2.35 & -0.55 & 3.526 & 0.09 & 1.6 & 0.70 & $\mathrm{E}$ & $77.2 \pm 14.7$ & 1.07 & .. & Dec 98 \\
\hline 1040 & $\mathrm{C}$ & 99 & 13.6 & 2.66 & -0.31 & 3.518 & 0.20 & 0.6 & $\ldots$ & $\ldots$ & $11.7 \pm 2.1$ & $\ldots$ & .. & Dec 97 \\
\hline 1042 & $\mathrm{C}$ & 99 & 16.0 & 2.70 & -1.08 & 3.483 & 0.55 & 0.0 & $\ldots$ & $\ldots$ & ........... & $\ldots$ & .. & Dec 97 \\
\hline 1044 & $\mathrm{C}$ & 87 & 14.6 & 3.30 & -0.54 & 3.500 & 0.23 & -1.5 & 2.98 & $\mathrm{E}$ & $\ldots \ldots \ldots$ & $\ldots$ & .. & Dec 98 \\
\hline 1047 & $\mathrm{C}$ & 99 & 14.2 & 2.84 & -0.51 & 3.509 & -0.03 & 0.6 & $\ldots$ & $\ldots$ & $16.3 \pm 2.8$ & $\ldots$ & .. & Dec 97 \\
\hline 1049 & $\mathrm{C}$ & 95 & 11.8 & 1.57 & 0.30 & 3.612 & 0.10 & 2.2 & $\ldots$ & $\ldots$ & $18.6 \pm 1.2$ & $\ldots$ & .. & Jan 97 \\
\hline
\end{tabular}


Table 2. Gliese Stars Used as Narrow-lined Spectral Templates

\begin{tabular}{ccccc}
\hline \hline Gliese\# & Spectral Type & $\begin{array}{c}V \\
(\mathrm{mag})\end{array}$ & $\begin{array}{c}\text { RA (1950) } \\
(\mathrm{h} \mathrm{m} \mathrm{s})\end{array}$ & $\begin{array}{c}\text { Dec (1950) } \\
(\mathrm{deg}, ")\end{array}$ \\
\hline 75 & $\mathrm{~K} 0 \mathrm{~V}$ & 5.6 & 014406 & +633624 \\
144 & $\mathrm{~K} 2 \mathrm{~V}$ & 3.7 & 033034 & -093736 \\
114 & $\mathrm{~K} 7 \mathrm{~V}$ & 8.9 & 024749 & +153036 \\
$15 \mathrm{~A}$ & $\mathrm{M} 1.5 \mathrm{~V}$ & 8.1 & 001531 & +434424 \\
411 & $\mathrm{M} 2 \mathrm{~V}$ & 7.5 & 110037 & +361818 \\
\hline
\end{tabular}


Table 3. Mean Radii

\begin{tabular}{lrccccc}
\hline \hline Bin & $\log L / L \odot$ range & $\log T_{\text {eff range }}$ & $\mathrm{N}$ & $\begin{array}{c}\langle R \sin (i)\rangle \\
\left(\mathrm{R}_{\odot}\right)\end{array}$ & $\begin{array}{c}\frac{\langle R \sin (i)\rangle}{0.79} \\
\left(\mathrm{R}_{\odot}\right)\end{array}$ & $\begin{array}{c}R \text { for bin center } \\
\left(\mathrm{R}_{\odot}\right)\end{array}$ \\
\hline A1 & $1.11-1.48$ & $3.682-3.730$ & 3 & $3.53 \pm 0.72$ & $4.49 \pm 0.92$ & 5.75 \\
$\mathrm{~A} 2$ & $0.74-1.11$ & $3.682-3.730$ & 5 & $2.85 \pm 0.32$ & $3.62 \pm 0.41$ & 3.75 \\
& & & & & & \\
B1 & $1.11-1.48$ & $3.634-3.682$ & 2 & $3.86 \pm 0.12$ & $4.91 \pm 0.15$ & 7.17 \\
B2 & $0.74-1.11$ & $3.634-3.682$ & 3 & $3.34 \pm 0.10$ & $4.25 \pm 0.13$ & 4.68 \\
B4 & $0.00-0.37$ & $3.634-3.682$ & 3 & $1.18 \pm 0.37$ & $1.51 \pm 0.47$ & 2.00 \\
& & & & & \\
C3 & $0.37-0.74$ & $3.586-3.634$ & 2 & $2.64 \pm 0.53$ & $3.36 \pm 0.67$ & 3.81 \\
C4 & $0.00-0.37$ & $3.586-3.634$ & 3 & $1.16 \pm 0.36$ & $1.48 \pm 0.45$ & 2.49 \\
& & & & & & \\
D4 & $0.00-0.37$ & $3.538-3.586$ & 8 & $1.52 \pm 0.16$ & $1.94 \pm 0.21$ & 3.11 \\
D5 & $-0.37-0.00$ & $3.538-3.586$ & 10 & $1.23 \pm 0.11$ & $1.56 \pm 0.14$ & 2.03 \\
& & & & & & \\
E5 & $-0.37-0.00$ & $3.490-3.538$ & 10 & $1.08 \pm 0.11$ & $1.37 \pm 0.14$ & 2.53 \\
E6 & $-0.74--0.37$ & $3.490-3.538$ & 10 & $0.92 \pm 0.08$ & $1.17 \pm 0.10$ & 1.65 \\
\hline
\end{tabular}

